Pacific

Journal of

Mathematics

\title{
LOCAL COLLAPSING THEORY
}

F.T. FARRELl AND L.E. JoneS 


\title{
LOCAL COLLAPSING THEORY
}

\author{
F.T. FARRELl AND L.E. JONES
}

The purpose of this paper is to introduce a variant of the geometric collapsing theory for Riemannian manifolds contained in the joint work of J. Cheeger, K. Fukaya and M. Gromov (henceforth called the "C-F-G-theory"). The authors make important use of this variant geometric collapsing theory in Farrell and Jones, 1998(2), to develop a theory for collapsing foliated Riemannian manifolds and then to prove topological rigidity for certain aspherical manifolds in Farrell and Jones, 1998(1).

\section{Introduction.}

Cheeger-Fukaya-Gromov have developed in [4] and in their earlier papers [5], $[\mathbf{6}],[\mathbf{1 0}]$ and $[\mathbf{1 1}]$ a theory of "collapsing" Riemannian manifolds (henceforth referred to as the C-F-G-theory). The purpose of this paper is to introduce a variant of the C-F-G-theory which is needed by the authors in [9] for developing their theory of "collapsing" foliated Riemannian manifolds, and in [8] for proving topological rigidity for $A$-regular complete non-positively curved Riemannian manifolds of dimension greater than four.

Recall that a Riemannian manifold $(M, g)$ is said to be $A$-regular for some sequence of positive numbers $A=\left\{A_{i}\right\}$ if we have that

$$
\left|\nabla^{i} R\right| \leq A_{i}
$$

for all $i$, where the indices $i$ vary over all the natural numbers and $\nabla^{i} R$ is the $i$-th covariant derivative of the curvature tensor (cf. [4, p. 334]). Note that the 0 -th condition means that the sectional curvature values are pinched; i.e., bounded away from $\pm \infty$. The C-F-G-theory, as well as its variant developed in this paper, both apply to any complete $A$-regular Riemannian manifold $(M, g)$. In the rest of this paper we shall always assume that $(M, g)$ is a complete $A$-regular Riemannian manifold.

The main results of C-F-G-theory are reviewed in Appendix 2 below (cf. A.2.2 and A.2.3). These results tell us that each sufficiently small piece of a complete $A$-regular Riemannian manifold $(M, g)$ has a neighborhood in $M$ denoted by $V$ which is equal to the orbit space $\Lambda \backslash \hat{V}$ of a free and properly discontinuous group action $\Lambda \times \hat{V} \rightarrow \hat{V}$ by isometries; the Riemannian manifold $\hat{V}$ (which is just a covering space of $V$ ) has injectivity radius at all 
points greater than $\delta$, where $\delta>0$ depends only on the $\left\{A_{i}\right\}$ and $\operatorname{dim} M$; and the action $\Lambda \times \hat{V} \rightarrow \hat{V}$ extends to an effective group action $H \times \hat{V} \rightarrow \hat{V}$ by a Lie group $H$ which is both virtually connected and virtually nilpotent, and which contains $\Lambda$ as a co-compact discrete subgroup.

In the remainder of this section we will formulate the main results of this paper (cf. Theorems 0.3 and 0.5 below); and give a brief outline of the paper.

In 0.1-0.3 below let $r: U \rightarrow B$ denote a smooth mapping from a submanifold $U \subset M$ (with $\partial U=\phi$ ) onto a Riemannian manifold $\left(B, g_{B}\right)$.

0.1. The curvature $K(r ; M)$. We let $K(r ; M)$ denote the glb (greatest lower bound) of all numbers $\sigma>0$ which satisfy the following properties for any smooth path $f:[0,1] \rightarrow U$ :

(a) $\Theta\left(T U_{f(1)}, P_{f}\left(T U_{f(0)}\right)\right)<\sigma(\operatorname{length}(f))$;

(b) $\left\|D r_{f(1)}-P_{f}\left(D r_{f(0)}\right)\right\|<\sigma($ length $(f))$.

Here $\Theta(V, W)$ denotes the angular distance between planes $V$ and $W$ (i.e., the maximum of the angular distance from each vector of $V$ to $W$ and from each vector of $W$ to $V)$; and $P_{f}$ denotes parallel translation along $f$ in $(M, g)$ in Part (a). In Part (b) $P_{f}\left(D r_{f(0)}\right)$ is defined to be the composite map

$$
P_{r \circ f} \circ D r_{f(0)} \circ \pi \circ P_{\bar{f}}: T U_{f(1)} \rightarrow T B_{r \circ f(1)},
$$

where $P_{\bar{f}}$ is a parallel translation in $(M, g)$ along the path $\bar{f}(t)=f(1-t)$, and $\pi: T M_{f(0)} \rightarrow T U_{f(0)}$ is orthogonal projection, and $P_{r \circ f}$ denotes parallel translation along $r \circ f$ in $\left(B, g_{B}\right)$. Note that $K(r ; M)$ depends on $U$ and $(B, g)$ as well as on $r$ and $(M, g)$.

0.2. Infranil cores. An infranil core for $(M, g)$ consists of a smooth submanifold $U \subset M$ with empty boundary, Riemannian manifold $\left(B, g_{B}\right)$, and a smooth bundle projection $r: U \rightarrow B$, such that the following properties hold:

0.2.1. (a) The fibers of $r$ are infranil manifolds; in particular they are closed aspherical manifolds with $\pi_{1}$ an infranil group. (Recall that an "infranil manifold" is a double coset space $\Gamma \backslash G / K$ where $G$ is a Lie group which is both virtually connected and virtually nilpotent, $K$ is a maximal compact subgroup and $\Gamma$ is a torsion free, co-compact, discrete subgroup of $G$.)

(b) $B$ is an open ball centered at the origin of some Euclidean space $\mathbf{R}^{n}$; and $g_{B}$ is the Euclidean metric.

(c) $U \subset M$ has a well-defined tubular neighborhood $E$ of radius equal $\operatorname{radius}(B)$. That is the exponential map exp : $T_{\delta}^{\perp}(U) \rightarrow M$ is a smooth embedding with image equal $E$, where $\delta=\operatorname{radius}(B)$ and where $T_{\delta}^{\perp} U$ denotes the set of all $v \in T M_{\mid U}$ which are perpendicular to $U$ and which satisfy $|v|<\delta$.

The radius of an infranil core $r$ is defined to be the radius of $B$ if $B \neq$ point. If $B=$ point, then the radius of $r$ is a fixed number $\delta>0$ such that 
$U$ has a well-defined tubular neighborhood in $M$ of radius $\delta$ (as described in $0.2 .1(\mathrm{c}))$.

Remark 0.2.1.1. In the preceding definition of "infranil core", by the boundary of $U$ we mean the manifold boundary. So typically $U$ is a noncompact manifold without manifold boundary. The closure of $U$ in $M$, denoted $C(U)$, is typically a compact manifold with boundary; and the map $r: U \rightarrow B$ typically extends to a fiber bundle map $C(U) \rightarrow C(B)$ where $C(B)$ denotes the closure of $B$ in $\mathbf{R}^{n}$.

Example 0.2.1.2. Let the double coset space $\Gamma \backslash G / K$ denote an infranil manifold. Let $h: \Gamma \rightarrow O(k)$ denote a representation for $\Gamma$ into the group of orthogonal transformations of $\mathbf{R}^{k}$; and let $\Gamma \times(G / K) \times \mathbf{R}^{k} \rightarrow(G / K) \times \mathbf{R}^{k}$ denote the diagonal action. Set

$$
M=\left(\Gamma \backslash(G / K) \times \mathbf{R}^{k}\right) \times \mathbf{R}^{l} \text { and } U=(\Gamma \backslash(G / K) \times 0) \times B^{l}
$$

where $B^{l}$ denotes the open ball of radius $\delta>0$ centered at the origin of $\mathbf{R}^{l}$. Define

$$
r: U \rightarrow B
$$

to be the standard projection $(\Gamma \backslash(G / K) \times 0) \times B^{l} \rightarrow B^{l}$. Then $r: U \rightarrow B$ is an infranil core for $(M, g)$ having radius $\delta$ for any Riemannian metric $g$ on $M$ with respect to which $U$ has a tubular neighborhood of radius $\delta$ in $(M, g)$ (cf. 0.2.1(c)). (In Remark 0.2.2.1 below we construct such a metric g.)

Now let $r: U \rightarrow B$ denote an arbitrary infranil core for $(M, g)$ of radius $\delta$. We shall say that $r: U \rightarrow B$ is $(\epsilon, \theta)$-rigid, for numbers $\epsilon, \theta>0$, if the following properties hold:

0.2.2. (a) $K(r ; M) \leq \epsilon\left(\delta^{-1}\right)$.

(b) diameter $\left(r^{-1}(x)\right)<\epsilon \delta$, for all $x \in B$. (This refers to the diameter of the manifold $r^{-1}(x)$ with respect to its Riemannian metric inherited from $(M, g)$.)

(c) For any $w \in T U$ which is perpendicular to the fibers of $r$ we have that

$$
(1-\epsilon)|w| \leq|\operatorname{Dr}(w)| \leq(1+\epsilon)|w| .
$$

(d) For each $v \in T M_{\mid U}$ which is perpendicular to $U$ there is a smooth path $f:[0,1] \rightarrow U$, which starts and ends at the foot of $v$, and which satisfies

$$
\text { length }(f)<\epsilon \delta \text { and } \theta<\Theta\left(v, P_{f}(v)\right) \text {. }
$$

Here $\Theta\left(v, P_{f}(v)\right)$ denotes the angular distance between vectors, and $P_{f}$ denotes parallel translation in $(M, g)$ along $f$. 
Remark 0.2.2.1. The question arises as to when the example constructed in 0.2.1.2 is $(\epsilon, \theta)$-rigid? To answer this we first construct a specific Riemannian metric $g$ on the manifold $M=\left(\Gamma \backslash(G / K) \times \mathbf{R}^{k}\right) \times \mathbf{R}^{l}$ of 0.2.1.2. Let $\langle,\rangle_{G / K}$ denote any Riemannian metric on $G / K$ with respect to which $\Gamma \times(G / K) \rightarrow G / K$ is an action by isometries; and let $\langle,\rangle_{\mathbf{R}^{k}}$ and $\langle,\rangle_{\mathbf{R}^{l}}$ denote the Euclidean metrics on $\mathbf{R}^{k}$ and $\mathbf{R}^{l}$. Define a Riemannian metric $g$ on $M$ to be the quotient of the product of $\langle,\rangle_{G / K}$ and $\langle,\rangle_{\mathbf{R}^{k}}$ and $\langle,\rangle_{\mathbf{R}^{l}}$. Note that (with respect to the metric $g$ just constructed) we have that

$$
K(r ; M)=0 \text {; }
$$

so Property 0.2.2(a) holds. Property 0.2.2(b) will hold iff

$$
\operatorname{diameter}(\Gamma \backslash G / K)<\epsilon \delta
$$

with respect to the metric induced on $\Gamma \backslash G / K$ by $\langle,\rangle_{G / K}$. By construction we have that

$$
|\operatorname{Dr}(w)|=|w|
$$

for any vector $w \in T U$ which is perpendicular to the fibers of $r$; so Property 0.2.2(c) holds. Finally, we note that Property $0.2 .2(\mathrm{~d})$ holds iff for each $v \in$ $\mathbf{R}^{k}$ and each $x \in G / K$ there is $g \in \Gamma$, and a smooth path $p:[0,1] \rightarrow G / K$ which starts at $x$ and ends at $g(x)$, which satisfy

$$
\operatorname{length}(p)<\epsilon \delta \text { and } \theta<\Theta(v, h(g)(v)) \text {. }
$$

Here $\operatorname{length}(p)$ is measured with respect to $\langle,\rangle_{G / K}, h: \Gamma \rightarrow O(k)$ is the representation of $\Gamma$ given in 0.2.1.2, and $\Theta($,$) is the angular distance in$ $\mathbf{R}^{k}$.

Existence Theorem 0.3. There is an integer $\eta>0$ and a number $\theta \in$ $(0,1)$ which depend only on $\operatorname{dim} M$. For any given $\epsilon \in(0,1)$ there is an arbitrarily small decreasing sequence of positive numbers $\delta_{1}>\delta_{2}>\delta_{3}>\ldots$ having arbitrarily small quotients $\delta_{j} / \epsilon \delta_{j-1}$; each $\delta_{j}$ depends only on $j, \epsilon$, $\operatorname{dim} M,\left\{\delta_{1}, \delta_{2}, \ldots, \delta_{j-1}\right\}$ and the $A=\left\{A_{i}\right\}$ of $(0.0)$. There is, for each integer $n \geq 0$ and for each $p \in M$, an infranil core $r: U \rightarrow B$ for $(M, g)$ and a point $p^{\prime} \in U$ which satisfy the following properties:

(a) The radius of $r$ is equal $\delta_{c}$ for some $c \in(n, n+\eta)$; $c$ depends on $p$ as well as on $n$.

(b) $r$ is $(\epsilon, \theta)$-rigid.

(c) $d\left(p, p^{\prime}\right)<\epsilon \delta_{c}$ and $\left|r\left(p^{\prime}\right)\right|=0$.

Remark 0.3.1. $U$ may be thought of as a stratum of lowest dimension provided by the C-F-G-theory for collapsing a small piece of $M$; $U$ is "collapsed" along the fibers of $r: U \rightarrow B$. This idea will be made precise when Theorem 0.3 is proven in $\S 3$ below. Note that for some $p \in M$ there might not be any collapsing at all: If the radius of injectivity at $p$ is greater than $\delta_{1}$ then we may choose $U$ to be the open ball of radius $\delta_{n}$ centered at $p$ in 
$M, B$ to be the open ball of radius $\delta_{n}$ centered at the origin in $T M_{p}$ and $r: U \rightarrow B$ to be the inverse of the exponential map.

Remark 0.3.2. The role of the integer $n$ in Theorem 0.3 is not very apparent; it becomes more apparent when the authors apply Theorem 0.3 in reference [9]. The reader should try to understand Theorem 0.3 and its proof for the special case $n=0$; the general case $n \geq 0$ is handled in the same way.

0.4. Thickened infranil cores. Let $r: U \rightarrow B$ denote an $(\epsilon, \theta)$-rigid infranil core of radius $\delta>0$. Note that Property 0.2.1(c) assures us that $U$ has a well-defined open tubular neighborhood $E$ of radius $\delta$ in $(M, g)$ and that the orthogonal projection $\rho: E \rightarrow U$ is a well-defined bundle projection map. (The orthogonal projection $\rho: E \rightarrow U$ is just the composite of the usual fiber bundle projection $T_{\delta}^{\perp}(U) \rightarrow U$ with $\exp ^{-1}: E \rightarrow T_{\delta}^{\perp}(U)$ of 0.2.1(c).) Define $s: E \rightarrow B$ to be the composite $r \circ \rho$; and define $t: E \rightarrow \mathbf{R}$ by $t(x)=d(x, \rho(x))$ for all $x \in E$. The pair of maps $(s, t)$ represent a thickened infranil core of diameter $\delta$ which is the "thickening" for the infranil core $r$. For each $\delta^{\prime} \in(0, \delta]$ we let $B\left(\delta^{\prime}\right)$ denote the open ball of radius $\delta^{\prime}$ centered at the origin of $B$, and we set

$$
E\left(\delta^{\prime}\right)=s^{-1}\left(B\left(\delta^{\prime}\right)\right) \cap t^{-1}\left(\left[0, \delta^{\prime}\right)\right) \text { and } U\left(\delta^{\prime}\right)=r^{-1}\left(B\left(\delta^{\prime}\right)\right) .
$$

Comparison Theorem 0.5. Given $\theta>0$ we let $\epsilon, \delta>0$ denote sufficiently small numbers, where how small is sufficient depends only on $\theta, \operatorname{dim} M$ and on the numbers $A=\left\{A_{i}\right\}$ of (0.0) above. Let $r_{i}: U_{i} \rightarrow B_{i}, i=1,2$, denote $(\epsilon, \theta)$-rigid infranil cores both of radius $\delta$. If $E_{1}(\delta / 9) \cap E_{2}(\delta / 9) \neq \phi$ then there is an isometry $I: \mathbf{R}^{k} \rightarrow \mathbf{R}^{k}$ (where $k=\operatorname{dim} B_{1}$ ) such that the following properties hold for each $x \in E_{1} \cap E_{2}$ :

(a) $\operatorname{dim} U_{1}=\operatorname{dim} U_{2}$ and $B_{1}=B_{2}$.

(b) $\left|t_{1}(x)-t_{2}(x)\right|<\mathcal{O}(\epsilon) \delta$.

(c) $\left|I \circ s_{1}(x)-s_{2}(x)\right|<\mathcal{O}(\epsilon) \delta$.

Remark 0.5.1. The notation " $\mathcal{O}(\epsilon)$ " appearing in the inequalities of $0.5(\mathrm{~b})$ and (c) means that there is a $C^{\infty}$-function $g: \mathbf{R} \rightarrow \mathbf{R}$ with $g(0)=0$, which is independent of the infranil cores $r_{1}, r_{2}$ and of the numbers $\epsilon, \delta$, such that when $\mathcal{O}(\epsilon)$ is replaced by the number $|g(\epsilon)|$ then the resulting inequality is actually true.

Remark 0.5.2. Suppose that all the hypotheses of Theorem 0.5 are still in effect. Then Properties 0.5(a)-(c) are equivalent to the following three properties. For each $x \in U_{2} \cap E_{1}$ let $f_{x}:[0,1] \rightarrow \rho_{1}^{-1}\left(\rho_{1}(x)\right)$ denote the geodesic with $f_{x}(0)=x, f_{x}(1)=\rho_{1}(x)$; and let $\mathcal{G}_{i}$ denote the foliation of $U_{i}$ by the fibers of $r_{i}$.

(a) length $\left(f_{x}\right)<\mathcal{O}(\epsilon) \delta$.

(b) $\Theta\left(P_{f_{x}}\left(T U_{2 \mid x}\right), T U_{1 \mid \rho_{1}(x)}\right)<\mathcal{O}(\epsilon)$. 
(c) $\Theta\left(P_{f_{x}}\left(T \mathcal{G}_{2 \mid x}\right), T \mathcal{G}_{1 \mid \rho_{1}(x)}\right)<\mathcal{O}(\epsilon)$.

This remark will be proven in Section 1.

This completes the statement of our main results of this paper.

The paper is organized as follows:

$\S 1$ : In this section we focus on the Comparison Theorem proving Theorem 0.5 and Remark 0.5.2.

$\S 2$ : In this section we formulate and prove a number of geometric lemmas which will be used in the proof of Theorem 0.3. These lemmas concern the isometric actions of connected nilpotent Lie groups which have "local angle control" (cf. 2.1). Lemma 2.8 describes the properties of 1-parameter subgroups of Lie groups which act with local control on themselves.

$\S 3$ : In this section we complete the proof of Theorem 0.3. We begin with a local collapsing structure for $M$ near the point $p$ (of 0.3 ) which is provided by C-F-G-theory [4]. We then use the lemmas of $\S 2$ to modify this collapsing structure so that we have better geometric control over the modified one; now the desired infranil core $U$ of 0.3 is taken to be the "thrice repeating layer" of this modified collapsing structure (cf. 3.5.1).

We have also included two appendices at the end of this paper. In Appendix 1 we discuss $A$-regularity of a Riemannian manifold $(M, g)$ in terms of local (normal) coordinate systems for $M$; these results are used both in $\S 1$ and $\S 3$ of this paper. It is recommended that this appendix be consulted before reading Section 1. In Appendix 2 we formulate the results from CF-G-theory (cf. [4]) which are needed to prove Theorem 0.3 in $\S 3$ of this paper. In particular we prove Theorems A.2.3 and A.2.5 which describe the group action on the fibration of orthonormal frames. This appendix is best read after reading $\S 1$ and $\S 2$ and before reading $\S 3$.

\section{Proof of Theorem 0.5 and Remark 0.5.2.}

It is recommended that the reader consult Appendix 1 before reading this section.

Proof of Remark 0.5.2. We leave as an exercise for the reader to check that Properties 0.5(a)-(c) (together with $(\epsilon, \theta)$-rigidity for $r_{1}, r_{2}$, and the inequality $\left.E_{1}(\delta / 9) \cap E_{2}(\delta / 9) \neq \phi\right)$ imply Properties 0.5.2(a)-(c).

To see that Properties 0.5.2(a)-(c) (together with $(\epsilon, \theta)$-rigidity for $r_{1}, r_{2}$, and the inequality $\left.E_{1}(\delta / 9) \cap E_{2}(\delta / 9) \neq \phi\right)$ imply Properties $0.5(\mathrm{a})$-(c) we argue as follows: First note that $0.5(\mathrm{~b})$, and the first equality of $0.5(\mathrm{a})$, follow immediately from 0.5.2(a) and (b). The second equality of $0.5(\mathrm{a})$ is equivalent to $\operatorname{dim} B_{1}=\operatorname{dim} B_{2}$ (since $r_{1}$ and $r_{2}$ are assumed to have the same radius); and this last equality follows from 0.5.2(a)-(c) applied at any $x \in r_{1}^{-1}(0)$. 
Towards verifying Property $0.5(\mathrm{c})$ we first need a candidate for the isometry $I: \mathbf{R}^{k} \rightarrow \mathbf{R}^{k}$ of $0.5(\mathrm{c})$. Choose $q \in r_{1}^{-1}(0)$, and let $V \subset T\left(U_{1}\right)_{q}$ denote the subspace of all vectors which are perpendicular to $r_{1}^{-1}(0)$ at $q$. Note that it follows from 0.2.2 (as applied to $r_{1}$ ) that $D r_{1}: V \rightarrow T\left(B_{1}\right)_{0}$ is a linear isomorphism which satisfies

(i) $(1-\epsilon)|v| \leq\left|D r_{1}(v)\right| \leq(1+\epsilon)|v|$

for all $v \in V$. It follows from 0.5.2(a)-(c), and from 0.2.2 (as applied to $r_{2}$ ), and from the inequality $E_{1}(\delta / 9) \cap E_{2}(\delta / 9) \neq \phi$, that $D s_{2}: V \rightarrow T\left(B_{2}\right)_{s_{2}(q)}$ is a linear isomorphism which satisfies

(ii) $(1-\mathcal{O}(\epsilon))|v| \leq\left|D s_{2}(v)\right| \leq(1+\mathcal{O}(\epsilon))|v|$

for all $v \in V$. (In verifying this last inequality the reader should recall that $\delta>0$ is chosen small relative to the $\left\{A_{i}\right\}$ of (0.0).) Recall that $B_{1}=B_{2}$ (cf. $0.5(\mathrm{a})$ ), and that $B_{1}$ is an open ball of radius $\delta$ centered at the origin of $\mathbf{R}^{k}$; in what follows we will identify $T\left(B_{1}\right)_{0}$ with $\mathbf{R}^{k}$ via the Euclidean exponential map, and we shall identify $T\left(B_{2}\right)_{s_{2}(q)}$ with $T\left(B_{1}\right)_{0}$ via Euclidean parallel translation. Then it follows from (i) and (ii) that we may choose a linear isometry $L: \mathbf{R}^{k} \rightarrow \mathbf{R}^{k}$ so that

(iii) $\left\|L-D s_{2} \circ\left(D r_{1} \mid V\right)^{-1}\right\|<\mathcal{O}(\epsilon)$.

Now we define an isometry $I: \mathbf{R}^{k} \rightarrow \mathbf{R}^{k}$ as follows:

(iv) $I(0)=s_{2}(q)$ and $D I=L$.

Note that it follows from (i)-(iv) (see also 0.5.2(a)-(c)) that:

(v) $I \circ s_{1}(q)=s_{2}(q)$ and $\left\|D\left(I \circ s_{1}\right)_{q}-D\left(s_{2}\right)_{q}\right\|<\mathcal{O}(\epsilon)$.

Recall that $K(s: M, \delta)$ is defined preceding the statement of Theorem A.1.6 in Appendix 1. By applying Theorem A.1.6 (from the Appendix) to both $s_{1}$ and $s_{2}$ we may deduce that

(vi) $K\left(s_{1} ; M, \delta\right)<\mathcal{O}(\epsilon) \delta^{-1}$ for $i=1,2$.

Now Property 1.5(c) follows directly from (v) and (vi).

This completes the Proof of Remark 0.5.2.

Proof of Theorem 0.5. Let $f: \mathbf{R}^{m} \rightarrow M$ denote a map which is just the composition of a linear isometry $\mathbf{R}^{m} \rightarrow T M_{q_{1}}$ with the exponential map $\exp : T M \rightarrow M$, where $q_{1} \in r_{1}^{-1}(0)$. We note that for $\delta>0$ sufficiently small the restricted map $f: B_{10 \delta}^{m} \rightarrow M$ is a smooth immersion, where for any $x>0$ we let $B_{x}^{m}$ denote the open ball of radius $x$ centered at the origin of $\mathbf{R}^{m}$. (How small is sufficient for $\delta$ here depends only on the $\operatorname{dim} M$, $A=\left\{A_{i}\right\}$; see Corollary A.1.2 in Appendix 1 for further details.) For each $i=1,2$ we set

$$
\hat{U}_{i}=f^{-1}\left(U_{i}\right) \cap B_{10 \delta}^{m} \text { and } \hat{\mathcal{G}}_{i}=f^{-1}\left(\mathcal{G}_{i}\right) \cap B_{10 \delta}^{m} .
$$

Note that Properties 0.5(a)-(c) follow immediately from 0.2.2(a)-(c) (as applied to each of $r_{1}, r_{2}$ ), from 0.5 .2 , and from the following claim: In the 
following claim the manifold $B_{10 \delta}^{m}$ is equipped with the Riemannian structure $f^{*}(g)$, and we denote by $P_{u}()$ the parallel translation along a path $u:[0,1] \rightarrow B_{10 \delta}^{m}$ with respect to $f^{*}(g)$, and we denote by $\Theta($,$) the angular$ distance with respect to $f^{*}(g)$.

Claim 1.1. For each $x \in \hat{U}_{2} \cap B_{2 \delta}^{m}$ there is a smooth path $u:[0,1] \rightarrow B_{10 \delta}^{m}$, with $u(1) \in \hat{U}_{1}$ and $u(0)=x$, so that (a) (b) and (c) hold:

(a) length $(u)<\mathcal{O}(\epsilon) \delta$.

(b) $\Theta\left(T \hat{U}_{1 \mid u(1)}, P_{u}\left(T \hat{U}_{2 \mid u(0)}\right)\right)<\mathcal{O}(\epsilon)$.

(c) $\Theta\left(T \hat{\mathcal{G}}_{1 \mid u(1)}, P_{u}\left(T \hat{\mathcal{G}}_{2 \mid u(0)}\right)\right)<\mathcal{O}(\epsilon)$.

By the hypothesis of 0.5 (that $E_{1}(\delta / 9) \cap E_{2}(\delta / 9) \neq \phi$ ), and since the Euclidean metric on $B_{10 \delta}^{m}$ is a close approximation to $f^{*}(g)$ (cf. A.1.2), we may choose $x_{i} \in \hat{U}_{i} \cap B_{\delta / 7}^{m}$ for $i=1,2$. Let $V_{i} \subset \mathbf{R}^{m}$ and $H_{i} \subset \mathbf{R}^{m}$ denote the vector subspaces obtained by parallel translating (in the Euclidean metric) the tangent planes $T \hat{U}_{i \mid x_{i}}$ and $T \hat{\mathcal{G}}_{i \mid x_{i}}$ to the origin of $\mathbf{R}^{m}$, and let $\Theta_{e}($, denote the Euclidean angular distance between subspaces of $\mathbf{R}^{m}$. Now we may deduce from results in Appendix 1 (cf. A.1.2 and A.1.4), and from the curvature restriction placed on $r$ by $0.2 .2(\mathrm{a})$, that the following linearized version of 1.1 is actually equivalent to 1.1 .

\section{Claim 1.2.}

(a) There is $y \in V_{1}+x_{1}$ such that $\left|x_{2}-y\right|<\mathcal{O}(\epsilon) \delta$.

(b) $\Theta_{e}\left(V_{1}, V_{2}\right)<\mathcal{O}(\epsilon)$.

(c) $\Theta_{e}\left(H_{1}, H_{2}\right) \leq \mathcal{O}(\epsilon)$.

Thus to complete the proof of 0.5 it will suffice to verify Claim 1.2. Towards this end we first wish to "linearize" Properties 0.2.2(a)-(d). Note that for each $x, y \in B_{\delta / 6}^{m}$ with $f(x)=f(y)$ there is a smooth embedding $h: B_{\delta / 6}^{m} \rightarrow B_{2 \delta / 3}^{m}$ and an isometry $\bar{h}: \mathbf{R}^{m} \rightarrow \mathbf{R}^{m}$ which satisfy the following properties (see Corollary A.1.3 in Appendix 1 for further details):

1.3. (a) $h(x)=y$; and for all $z \in B_{\delta / 6}^{m}$ we have that $f(z)=f \circ h(z)$.

(b) $h(0)=\bar{h}(0)$, and for all $z \in B_{\delta / 6}^{m}$ we have that $\left|D(h-\bar{h})_{\mid z}\right|<\mathcal{O}(\epsilon)$.

(c) $h\left(\hat{U}_{i} \cap B_{\delta / 6}^{m}\right) \subset \hat{U}_{i} \cap B_{2 \delta / 3}^{m}$.

Now the "linearized" versions of 0.2.2(a), (c) and (d) as applied to $r_{1}, r_{2}$ (which follow from 0.2.2(a)-(d) as applied to $r_{1}, r_{2}$, and from 1.3, and from A.1.1-A.1.3) can be formulated as follows. For each isometry $\bar{h}$ from 1.3 let $\bar{h}_{r}$ denote its rotational part.

1.4. (a) For each $v \in V_{i}$ which is perpendicular to $H_{i}$, and for each $h$ in 1.3 , we have that

$$
\Theta_{e}\left(v, \bar{h}_{r}(v)\right)<\mathcal{O}(\epsilon)
$$


(b) For each $w \in \mathbf{R}^{m}$ which is perpendicular to $V_{i}$ there is an $h$ from 1.3 such that

$$
\theta / 4<\Theta_{e}\left(w, \bar{h}_{r}(w)\right) .
$$

The linearized version of 0.2.2(b) (which follows from 0.2.2(a)-(d) as applied to $r_{1}, r_{2}$, and from 1.3, and from A.1.1-A.1.3) can be formulated as follows. Set $Q_{i}=H_{i}+x_{i}$ and let $d_{e}($, ) denote the Euclidean distance in the following:

1.5. (a) For each $y \in Q_{i} \cap B^{m}(\delta)$ and each $\bar{h}$ of 1.3 we have that

$$
d_{e}\left(\bar{h}(y), Q_{i}\right)<\mathcal{O}(\epsilon) \delta .
$$

(b) For each $y \in Q_{i} \cap B^{m}(\delta)$ there is a map $\bar{h}_{y, i}$ from 1.3 such that

$$
d_{e}\left(y, \bar{h}_{y, i}\left(x_{i}\right)\right)<\mathcal{O}(\epsilon) \delta .
$$

Now we can use 1.4 and 1.5 to verify 1.2 .

We begin by verifying $1.2(\mathrm{c})$. Let $u \in H_{1}$ be a unit vector and set $g(t)=$ $x_{1}+t u$ for $t \in \mathbf{R}$. It will suffice to verify the following inequality for some fixed $b \in(0, \delta)$ and all $t \in[-\delta / 9, \delta / 9]$ :

1.6. $b-\mathcal{O}(\epsilon) \delta<d_{e}\left(g(t), Q_{2}\right)<b+\mathcal{O}(\epsilon) \delta$.

For any $t \in[-\delta / 9, \delta / 9]$ it follows from the fact $x_{1} \in B_{\delta / 7}^{m}$ that $g(t) \in B_{\delta / 3}^{m}$. Thus we may apply 1.5 (b) (with $y=g(t)$ and $i=1$ ) to get a map $\bar{h}_{y, 1}$ satisfying

$$
d_{e}\left(g(t), \bar{h}_{y, 1}\left(x_{1}\right)\right)<\mathcal{O}(\epsilon) \delta .
$$

Let $x \in Q_{2}$ denote the orthogonal projection of $x_{1}$ onto $Q_{2}$. By applying 1.5 (a) to $x \in Q_{2}$ with $\bar{h}=\bar{h}_{y, 1}$ we get that

$$
d_{e}\left(\bar{h}_{y, 1}(x), Q_{2}\right)<\mathcal{O}(\epsilon) \delta
$$

and it follows from this last inequality, from the fact that $x-x_{1}$ is perpendicular to $Q_{2}$, and from the fact that $\bar{h}_{y, 1}$ is an isometry satisfying 1.5 (a) for $i=2$, that we have

$$
\left|x-x_{1}\right|-\mathcal{O}(\epsilon) \delta<d_{e}\left(\bar{h}_{y, 1}\left(x_{1}\right), Q_{2}\right)<\left|x-x_{1}\right|+\mathcal{O}(\epsilon) \delta .
$$

Now 1.6 follows from the first and third of the last three inequalities for $b=\left|x-x_{1}\right|$.

Now we can verify $1.2(\mathrm{~b})$. Let $H_{i}^{\perp}$ denote the orthogonal complement for $H_{i}$ in $V_{i}$, and let $V_{i}^{\perp}$ denote the orthogonal complement of $V_{i}$ in $\mathbf{R}^{m}$. For each unit length vector $u \in H_{1}^{\perp}$ choose $v \in V_{2}$ and $w \in V_{2}^{\perp}$ such that $u=v+w$; and choose $v_{1} \in H_{2}$ and $v_{2} \in H_{2}^{\perp}$ such that $v=v_{1}+v_{2}$. Since 1.2 (c) has already been verified, to complete the verification of $1.2(\mathrm{~b})$ it will 
suffice to show that $|w|<\mathcal{O}(\epsilon)$. Use 1.4(b) (as applied to $V_{2}$ ) to choose $\bar{h}_{r}$ such that

$$
\Theta_{e}\left(w, \bar{h}_{r}(w)\right)>\theta / 4 .
$$

By applying 1.4(a) to $u \in H_{1}^{\perp}$ we get that

$$
\Theta_{e}\left(u, \bar{h}_{r}(u)\right)<\mathcal{O}(\epsilon) .
$$

We deduce from 1.2(c), and from $1.4(\mathrm{a})$ as applied to $v_{2} \in H_{2}^{\perp}$, that

$$
\left|v_{1}\right|<\mathcal{O}(\epsilon) \text { and } \Theta_{e}\left(v_{2}, \bar{h}_{r}\left(v_{2}\right)\right)<\mathcal{O}(\epsilon) .
$$

Finally, by using these last few inequalities, and the triangle inequality, and the fact that

$$
|x| \Theta_{e}(x, y) / 4 \leq|x-y| \leq 4|x| \Theta_{e}(x, y)
$$

holds for any two vectors $x, y \in \mathbf{R}^{m}$ of the same length, we get that

$$
\mathcal{O}(\epsilon)>\left|u-\bar{h}_{r}(u)\right| \geq\left|w-\bar{h}_{r}(w)\right|-\left|v-\bar{h}_{r}(v)\right|>\theta|w| / 16-\mathcal{O}(\epsilon) .
$$

From which we deduce that $|w|<\mathcal{O}(\epsilon)$ as desired.

Before verifying 1.2(a) we first remark that 1.4(a) and 1.5(a) together imply that:

1.7. $\Theta_{e}\left(V_{i}, \bar{h}_{r}\left(V_{i}\right)\right)<\mathcal{O}(\epsilon)$

for all $\bar{h}$ from 1.3 and for $i=1,2$. Now to verify $1.2\left(\right.$ a) we set $x_{2}-x_{1}=$ $v+w$ where $v \in V_{1}$ and $w \in V_{1}^{\perp}$. It will suffice (by $1.2(\mathrm{~b})$ ) to show that $|w|<\mathcal{O}(\epsilon) \delta$. Use 1.4(b) (as applied to $V_{1}$ ) to choose $h$ as in 1.3 so that

$$
\Theta_{e}\left(w, \bar{h}_{r}(w)\right)>\theta / 4
$$

and use 1.5(a) (as applied to $x_{1}+v \in Q_{1}$ ) to get that

$$
d_{e}\left(\bar{h}\left(x_{1}+v\right), Q_{1}\right)<\mathcal{O}(\epsilon) \delta .
$$

Note that 1.7 , and the fact that $\bar{h}_{r}$ is an isometry, together imply that

$$
\Theta_{e}\left(\bar{h}_{r}(w), V_{1}^{\perp}\right)<\mathcal{O}(\epsilon) .
$$

Now by combining these last three inequalities with 1.2(b), and with the fact that $\bar{h}\left(x_{2}\right)=\bar{h}\left(x_{1}+v\right)+\bar{h}_{r}(w)$, we get that

$$
d_{e}\left(\bar{h}\left(x_{2}\right), Q_{2}\right)>\theta|w| / 20-\mathcal{O}(\epsilon) \delta .
$$

On the other hand by applying 1.5 (a) to $x_{2} \in Q_{2}$ we get that

$$
d_{e}\left(\bar{h}\left(x_{2}\right), Q_{2}\right)<\mathcal{O}(\epsilon) \delta .
$$

These last two inequalities imply that $|w|<\mathcal{O}(\epsilon) \delta$ as desired.

This completes the Proof of Theorem 0.5. 


\section{Preliminaries to the proof of Theorem 0.3 .}

In this section we formulate several lemmas which will be needed for the proof of Theorem 0.3 given in the next section. These lemmas are concerned with isometric actions of a connected Lie group on a Riemannian manifold which have "local angle control" (cf. 2.1).

Let $N, g_{N}$ denote a connected Riemannian manifold (not necessarily complete), and we let $G \times N \rightarrow N$ denote an isometric effective action of the connected nilpotent Lie group $G$. For any numbers $\alpha, \beta>0$ we shall say that this action has local angle control equal $(\alpha, \beta)$ at $x \in N$ if any path $f:[0,1] \rightarrow N$ which satisfies 2.1(a) and (b) also satisfies 2.1(c).

2.1. (a) There is a path $\phi:[0,1] \rightarrow G$ starting at the identity such that $f(t)=\phi(t)(x)$.

(b) length $(f) \leq \beta$.

(c) $\Theta\left(D g(v), P_{f}(v)\right) \leq \alpha$ for all $v \in T N_{x}$, where $g=\phi(1)$ and where $P_{f}$ denotes parallel translation along the path $f$.

We shall say that the action $G \times N \rightarrow N$ has local angle control equal $(\alpha, \beta)$ if it has such angle control at each of its points $x \in N$.

Remark 2.2. Let $H \times \hat{V} \rightarrow \hat{V}$ denote a smooth action of the Lie group $H$ by isometries of an $A$-regular Riemannian manifold $\hat{V}$. Let $\rho: E \rightarrow \hat{V}$ denote the bundle of orthonormal frames over $\hat{V}$ equipped with the canonical metric and let $H \times E \rightarrow E$ denote the canonical lifting of the action $H \times \hat{V} \rightarrow \hat{V}$ (see Appendix 2 for more details). We shall prove in Appendix 2 (cf. Theorem A.2.5) that the action $H \times E \rightarrow E$ has local angle control equal $(\lambda t, t)$ for any $t>0$, where $\lambda>1$ depends only on $\operatorname{dim} E, A_{0}$.

Now we will formulate six lemmas which are the main results of this section. The proofs for these lemmas are also given in this section. The first two of these lemmas are just refinements of 2.1. Recall that in Definition $2.1 \phi$ is not necessarily a one-parameter subgroup of $G$.

Lemma 2.3. Let $\alpha \in(0, \pi)$ and $\beta>0$. Suppose that $G \times N \rightarrow N$ does not have local angle control equal $(\alpha, \beta)$ at $x \in N$. Then there is a path $f: \mathbf{R} \rightarrow N$ and $a$ unit vector $v \in T N_{x}$ which satisfy the following properties:

(a) There is a one-parameter subgroup $\phi: \mathbf{R} \rightarrow G$ such that $f(t)=\phi(t)(x)$ for all $t \in \mathbf{R}$.

(b) length $\left(\left.f\right|_{[0,1]}\right) \leq \beta$.

(c) $\Theta\left(D g(v), P_{\left.f\right|_{[0,1]}}(v)\right)>\alpha$ where $g=\phi(1)$.

Lemma 2.4. Let $\alpha \in(0, \pi)$ and $\beta>0$. Suppose $G \times N \rightarrow N$ has local angle control equal $(\alpha, \beta)$, and let $f:[0,1] \rightarrow N$ and $\phi:[0,1] \rightarrow G$ be any smooth paths which satisfy Properties $2.1(\mathrm{a})$ and (b). Then for all $v \in T N_{f(0)}$, and 
for $g=\phi(1)$, we have that

$$
\Theta\left(D g(v), P_{f}(v)\right)<(\alpha / \beta) \text { length }(f) .
$$

Recall that the isotropy subgroup $G_{x} \subset G$ for the action $G \times N \rightarrow N$ at $x \in N$ is defined by $G_{x}=\{g \in G: g(x)=x\}$.

Lemma 2.5. Let $\alpha \in(0, \pi)$ and $\beta>0$, and suppose that $G \times N \rightarrow N$ has local angle control equal $(\alpha, \beta)$. Then for each $x \in N$ the isotropy group $G_{x}$ in $G$ is a discrete subgroup of $G$.

Note that 2.5 implies that the orbits of $G \times N \rightarrow N$ foliate $N$. In the following remarks and lemma we denote this foliation of $N$ by $\mathcal{G}$.

Remark 2.6(i). We shall say that $\mathcal{G}$ is a strongly Riemannian foliation if for any path $f:[0,1] \rightarrow L$ in a leaf $L \in \mathcal{G}$ there is a $\delta>0$ and a continuous map $F:[0,1] \times \mathbf{R}^{k} \rightarrow N$, where $k=\operatorname{dim} N-\operatorname{dim} \mathcal{G}$, which satisfies:

(a) $F(t, 0)=f(t)$ for all $t$; and $F \mid[0,1] \times v$ is path in a leaf $L_{v} \in \mathcal{G}$ for each $v \in \mathbf{R}^{k}$.

(b) Let $V_{i}$ denote the open $\delta$-ball centered at the origin of $T \mathcal{G}_{f(i)}^{\perp}$, for $i=0,1$, where $T \mathcal{G}^{\perp}$ denotes the orthogonal complement in $T N$ for $T \mathcal{G}$. Then the exponential map exp $: T N \rightarrow N$ maps $V_{i}$ diffeomorphically onto a submanifold $W_{i} \subset N$; and $F \mid i \times \mathbf{R}^{k}$ maps $i \times \mathbf{R}^{k}$ homeomorphically onto $W_{i}$.

(c) Let $h: 0 \times \mathbf{R}^{k} \rightarrow 1 \times \mathbf{R}^{k}$ be defined by $h(0, v)=(1, v)$. Then the composite map $\left(F \mid 1 \times \mathbf{R}^{k}\right) \circ h \circ\left(F \mid 0 \times \mathbf{R}^{k}\right)^{-1}: W_{0} \rightarrow W_{1}$ is an isometry.

Remark 2.6(ii). The curvature $K(\mathcal{G} ; N)$ for $\mathcal{G}$ in $\left(N, g_{N}\right)$ is defined to be the glb of all numbers $\sigma>0$ which satisfy the following inequality for any smooth path $f:[0,1] \rightarrow N$ :

$$
\Theta\left(T \mathcal{G}_{f(1)}, P_{f}\left(T \mathcal{G}_{f(0)}\right)\right)<\sigma(\text { length }(f)) .
$$

Lemma 2.6. Let $\alpha \in(0, \pi)$ and $\beta>0$, and suppose that $G \times N \rightarrow N$ has local angle control equal $(\alpha, \beta)$. Then $\mathcal{G}$ is a strongly Riemannian foliation of $\left(N, g_{N}\right)$; and the curvature $K(\mathcal{G} ; N)$ for $\mathcal{G}$ in $\left(N, g_{N}\right)$ satisfies $K(\mathcal{G} ; N)<$ $10^{6} \alpha / \beta$.

In our next lemma we assume that the Riemannian metric $g_{N}$ on $N$ is $A$-regular for some collection $A=\left\{A_{i}: i=1,2,3, \ldots\right\}$ of positive numbers. We let $\Gamma \subset G$ denote a torsion free discrete subgroup such that the restricted action $\Gamma \times N \rightarrow N$ is fixed point free and properly discontinuous. For each $t>0$ and $x \in N$ we let $\Gamma_{t, x} \subset \Gamma$ denote the subset of all $g \in \Gamma$ such that $d_{N}(x, g(x))<t$, and we let $\bar{\Gamma}_{t, x}$ denote the subgroup of $\Gamma$ generated by $\Gamma_{t, x}$.

Lemma 2.7. Let $B>0$ be as in A.2.3; there is a number $\tau>1$ which depends only on $B$ and on $\operatorname{dim} N$. Suppose that $G \times N \rightarrow N$ has local angle 
control equal $(\alpha, \beta)$, where $\alpha$ and $\beta$ are sufficiently small and $\beta<\alpha$ (how small is sufficient depends only on $A=\left\{A_{i}\right\}$ and on $\left.\operatorname{dim} N\right)$. For a given $x \in N$ we assume that $\left(N, g_{N}\right)$ has radius of injectivity greater than $10^{4} \tau B \beta$ at $x$; we also assume that for each $t \in(0, \beta)$ and each $g \in \Gamma_{t, x}$ there is a oneparameter subgroup $h_{g}: \mathbf{R} \rightarrow G$ with $g \in h_{g}([0,1])$ and $d_{N}\left(x, h_{g}(s)(x)\right)<B t$ for all $s \in[0,1]$. (And $h_{e}$ equal the trivial subgroup.) Then for any $t \in(0, \beta)$ there is a closed subgroup $G_{t, x} \subset G$ such that all of the following properties hold:

(a) If $h: \mathbf{R} \rightarrow G$ is any other one-parameter subgroup of $G$ which satisfies $g \in h([0,1])$ and $d_{N}(x, h(s)(x))<B t$ for all $s \in[0,1]$, then there is a number $a \in \mathbf{R}$ such that $h($ as $)=h_{g}(s)$ for all $s \in \mathbf{R}$.

(b) $G_{t, x}$ is the smallest connected Lie subgroup of $G$ which contains each one-parameter subgroup $h_{g}: \mathbb{R} \rightarrow G, g \in \Gamma_{t, x}$.

(c) Set $L_{t, x}=\left\{g(x): g \in G_{t, x}\right\}$. Then the quotient space $L_{t, x} / \bar{\Gamma}_{t, x}$ is compact and has diameter less than $\tau t$. (The diameter refers to the metric induced on $L_{t, x}$ by the restricted Riemannian structure $g_{N} \mid$ $T\left(L_{t, x}\right)$.)

(d) Suppose that $G_{t, x}=G_{2 \tau t, x}$ for $t \in(0, \beta / 2 \tau)$; and suppose that for $g \in G_{t, x}$ we have that $d_{N}(x, g(x))<t$. Then there is $g^{\prime} \in G_{t, x}$ with $g x=g^{\prime} x$, and there is a one-parameter subgroup $h_{g^{\prime}}: \mathbf{R} \rightarrow G_{t, x}$ with $g^{\prime} \in h_{g^{\prime}}(0,2 t)$ such that the path $f_{g^{\prime}}: \mathbf{R} \rightarrow N$ defined by $f_{g^{\prime}}(s)=$ $h_{g^{\prime}}(s)(x)$ has unit speed.

In our final lemma we let $g_{G}$ denote a left invariant Riemannian metric on $G$ with respect to which the (left multiplication) group action $G \times G \rightarrow G$ has local angle control equal $(\alpha, \beta)$.

Lemma 2.8. Given any sufficiently small $\alpha>0$ (how small is sufficient depends only on $\operatorname{dim} G$ ) and given any $\beta>0$, suppose that $G \times G \rightarrow G$ has local angle control equal to $(\alpha, \beta)$. If $\phi_{1}: \mathbf{R} \rightarrow G$ denotes a one-parameter subgroup which satisfies (a) then there is another one-parameter subgroup $\phi_{2}: \mathbb{R} \rightarrow G$ which satisfies (b)-(c).

(a) There are numbers $s, t>0$, with $t<\beta$, such that $d_{G}\left(\phi_{1}(0), \phi_{1}(s)\right)<t$.

(b) We have $\phi_{2}(s)=\phi_{2}(0)$.

(c) $d_{G}\left(\phi_{1}(u), \phi_{2}(u)\right)<(u / s) 4^{k} t$ for all $u \in[0, s]$, where $k=\operatorname{dim} G$.

Proof of Lemma 2.3. Set $G_{x}=\{g \in G: g(x)=x\}$; note that $G_{x}$ is a closed subgroup of $G$ and is thus a Lie group.

If $\operatorname{dim} G_{x}>0$ then choose any one-parameter subgroup $\psi: \mathbf{R} \rightarrow G_{x}$. For some $r>0$ we have that $\psi(r)$ rotates some unit vector $v \in T N_{x}$ by at an angle greater than $\alpha$. We define $\phi: \mathbf{R} \rightarrow G$ by $\phi(t)=\psi(t r)$. Note that $\phi$ satisfies Properties 2.3(a) and (b).

If $\operatorname{dim} G_{x}=0$, then the map $h: G \rightarrow N$ defined by $h(g)=g(x)$ is an immersion. Hence it induces a left invariant Riemannian metric $g_{G}$ on 
$G$. For each $v \in T G_{e}$ with $|v|=1$, let $\gamma_{v}: \mathbf{R} \rightarrow G$ be the one-parameter subgroup such that $\dot{\gamma}_{v}(0)=v$. Define a curve

$$
A_{v}: \mathbf{R} \rightarrow \operatorname{Iso}\left(T N_{x}\right)
$$

by setting $A_{v}(t)$ equal to the composition of parallel translation along $\gamma_{v} x$ from $T N_{x}$ to $T N_{\gamma_{v}(t) x}$ and $D\left(\gamma_{v}(-t)\right): T N_{\gamma_{v}(t) x} \rightarrow T N_{x}$. It is easily seen that $A_{v}$ is a one-parameter subgroup of $\operatorname{Iso}\left(T N_{x}\right) \simeq O(n)$ where $n=\operatorname{dim} N$. Hence, there is an orthonormal basis for $T N_{x}$ so that the matrix representing each $A_{v}(t), t \in \mathbf{R}$, is the blocked sum of the identity matrix of a certain size and $2 \times 2$ matrices of the form

$$
\left[\begin{array}{cc}
\cos (\theta t) & \sin (-\theta t) \\
\sin (\theta t) & \cos (\theta t)
\end{array}\right]
$$

Assuming that the conclusion of 2.3 is false, we see that $\theta \leq \alpha / \beta$ for each of these matrices (provided $\alpha \in(0, \pi)$ ). We consequently get the following estimate valid for each $g \in G$, for each one-parameter subgroup $\gamma_{v}$ where $|v|=1$, for each $u \in T N_{g x}$, and for each $t \in[0, \infty)$ :

2.3.1. $\Theta\left(D\left(g \gamma_{v}(t) g^{-1}\right)(u), P(u)\right) \leq \alpha t / \beta$,

where $P(u)$ denotes the parallel transport of $u$ along $g \gamma_{v} x$ to $T N_{g \gamma_{v}(t) x}$. Since each smooth path $\phi:[0,1] \rightarrow G$ can be approximated (as closely as we want) by a piecewise smooth path $\psi:[0,1] \rightarrow G$ where each piece of $\psi$ has the form

$$
t \mapsto g \gamma_{v}(t-c)
$$

restricted to an interval of the form $[c, a]$, estimate 2.3.1 yields the following more general estimate where $f(t)=\phi(t) x$ :

2.3.2. $\Theta\left(D g(v), P_{f}(v)\right) \leq(\alpha / \beta) \operatorname{length}(f)$.

In 2.3.2 we have that $v \in T N_{x}$ with $v \neq 0$ and $g=\phi(1)$. Now clearly 2.3.2 implies that $G \times N \rightarrow N$ has local angle control equal $(\alpha, \beta)$ at $x$, in direct contradiction of the assumption of 2.3.

This completes the Proof of Lemma 2.3.

Proof of Lemma 2.4. Note that the conclusion of this lemma has been verified in 2.3.2 of the preceding lemma under the hypothesis that the conclusion of 2.3 is false. Certainly the conclusion of 2.3 is false if the hypotheses of 2.4 hold.

This completes the Proof of Lemma 2.4.

Proof of Lemma 2.5. Note that each isotropy subgroup $G_{x} \subset G, x \in N$, is a closed Lie subgroup of $G$. If $G_{x}$ is not a discrete subgroup of $G$ for some $x \in N$ then the identity component $G_{x, e}$ of $G_{x}$ has dimension $\geq 1$. 
Choose a nontrivial one-parameter subgroup $\bar{\phi}: \mathbf{R} \rightarrow G_{x, e}$, and note that the following hold:

2.5.1. (a) $\bar{\phi}(t)(x)=x$ for all $t \in \mathbf{R}$.

(b) For some $s>0$ and some $v \in T N_{x}$ we have that

$$
\Theta(D(\bar{\phi}(s))(v), v)=\pi
$$

Now define $\phi:[0,1] \rightarrow G$ by $\phi(t)=\bar{\phi}(t s)$, where $s$ comes from 2.5.1(b). It follows from 2.5.1, and from the definition for $\phi$ just given, that $\phi$ does satisfy Properties 2.1(a) and (b) but does not satisfy Property 2.1(c) for any $\alpha<\pi$ in 2.1(c). This contradicts the hypothesis for 2.5 .

This completes the proof for Lemma 2.5.

Proof of Lemma 2.6. First we will verify that $\mathcal{G}$ is a strongly Riemannian foliation. Let $f:[0,1] \rightarrow L$ be a path in a leaf $L \in \mathcal{G}$. We may choose a path $\phi:[0,1] \rightarrow G$ which is related to $f$ as follows (cf. 2.5):

2.6.1. (a) $\phi(0)$ is the identity of the group $G$.

(b) $f(t)=\phi(t)(f(0))$ for all $t \in[0,1]$.

Let $V_{i}$ denote the open $\delta$-ball centered at the origin of $T \mathcal{G}_{f(i)}^{\perp}, i=0,1$, where $T \mathcal{G}^{\perp}$ denotes the orthogonal complement in $T N$ to $T \mathcal{G}$, and where $\delta>0$ is chosen sufficiently small to assure that the exponential map exp : $T N \rightarrow N$ is well-defined on $V_{i}$ and maps $V_{i}$ diffeomorphically onto a smooth submanifold $W_{i} \subset N$. Note it follows from 2.6.1(a) and (b), and from the fact that $G$ acts by isometries on $N$, that:

2.6.1. (c) $\phi(1)\left(W_{0}\right)=W_{1}$.

Choose a homeomorphism $F: 0 \times \mathbf{R}^{k} \rightarrow W_{0}$ which sends $(0,0) \in 0 \times \mathbf{R}^{k}$ to $\exp (0) \in W_{0}$, where $k=\operatorname{dim} W_{0}$. Extend $F \mid 0 \times \mathbf{R}^{k}$ to a map $F$ : $[0,1] \times \mathbf{R}^{k} \rightarrow N$ by setting:

2.6.1. (d) $F(t, v)=\phi(t)(F(0, v))$

for each $t \in[0,1]$. Note that Properties (a), (b) and (c) in Remark 2.6(i) are an immediate consequence of 2.6.1(a)-(d).

Now we shall verify the curvature condition for $\mathcal{G}$ stated in 2.6. Because $\mathcal{G}$ is a strongly Riemannian foliation, it will suffice (cf. Remark 2.6.4 at the end of this proof) to show that the following property holds:

2.6.2. Let $f:[0,1] \rightarrow L$ denote a smooth path with length $(f)<\beta$ in a leaf $L \in \mathcal{G}$; and let $P_{f}: T N_{f(0)} \rightarrow T N_{f(1)}$ denote parallel translation along 
$f$. Then we have that

$$
\Theta\left(T \mathcal{G}_{f(1)}, P_{f}\left(T \mathcal{G}_{f(0)}\right)\right)<(\alpha / \beta) \operatorname{length}(f) .
$$

Using Lemma 2.5 we may choose $\phi:[0,1] \rightarrow G$ starting at the identity such that $\phi(t)(x)=f(t)$ for all $t \in[0,1]$ and set $x=f(0)$. Now since $\phi$, $f$ satisfy Properties 2.1(a) and (b), and since $G \times N \rightarrow N$ has local angle control equal $(\alpha, \beta)$, it follows from Lemma 2.4 that $f:[0,1] \rightarrow N$ satisfies:

2.6.3. $\Theta\left(D g(v), P_{f}(v)\right)<(\alpha / \beta) \operatorname{length}(f)$

for any $v \in T N_{x}$ where $g=\phi(1)$. Since $D g\left(T \mathcal{G}_{f(0)}\right)=T \mathcal{G}_{f(1)}$, the inequality of 2.6.2 is a direct consequence of the inequality in 2.6.3.

This completes the proof for Lemma 2.6 (modulo the contents of Remark 2.6.4 below).

Remark 2.6.4. We will show in this remark that if the inequality of 2.6 .2 holds for all smooth paths $f:[0,1] \rightarrow N$ which are tangent to $\mathcal{G}$, then the inequality:

(i) $\Theta\left(T \mathcal{G}_{f(1)}, P_{f}\left(T \mathcal{G}_{f(0)}\right)\right)<10^{6}(\alpha / \beta) \operatorname{length}(f)$

holds for all smooth paths in $N$. To accomplish this it will suffice to verify that for any compact subset $C \subset N$, and for any sufficiently small number $\epsilon>0$ (how small is sufficient depends on $C$ ), a smooth path $f:[0,1] \rightarrow N$ satisfies (i) provided it satisfies:

(ii) $f(0)=p$ for some $p \in C$, and length $(f)=\epsilon$.

Let $x_{1}, x_{2}, \ldots, x_{n}$ denote the normal coordinates near $p$ with $p \sim(0,0$, $\ldots, 0)$, and let $g_{i, j} d x_{i} d x_{j}$ denote the coordinate expression for the Riemannian metric on $N$. Then $g_{i, j}(0, \ldots, 0)=\delta_{j}^{i}, \partial g_{i, j} / \partial x_{k}(0, \ldots, 0)=0$, and $\Gamma_{i, j}^{k}(0, \ldots, 0)=0$ for all $i, j, k$. Thus, for sufficiently small $\epsilon>0$ there is a number $\tau>0$, independent of $\epsilon$ and of $p \in C$, such that:

(iii) $\left|g_{i, j}(X)-\delta_{j}^{i}\right|<\tau \epsilon^{2}$ for all $X \in B_{10 \epsilon}^{n}$,

(iv) $\left|\Gamma_{i, j}^{k}(X)\right|<\tau \epsilon$ for all $X \in B_{10 \epsilon}^{n}$,

where for any number $t>0 B_{t}^{n}$ denotes the set of all points $X=\left(x_{1}, \ldots, x_{n}\right)$ in $\mathbf{R}^{n}$ with Euclidean length less than $t$. Let $d($,$) and \bar{d}($,$) denote the$ metrics on $B_{10 \epsilon}^{m}$ induced by $g_{i, j} d x_{i} d x_{j}$ and $\delta_{j}^{i} d x_{i} d x_{j}$ respectively; and for any smooth path $h:[0,1] \rightarrow B_{10 \epsilon}^{n}$ we let $P_{h}()$ and $\bar{P}_{h}($ ) denote parallel translation along $f$ induced by $g_{i, j} d x_{i} d x_{j}$ and $\delta_{j}^{i} d x_{i} d x_{j}$ respectively. We can deduce the following properties from (iii) and (iv), provided length $(h)<10 \epsilon$ :

(v) $|d(X, Y)-\bar{d}(X, Y)|<100 n^{2} \tau \epsilon^{3}$ for all $X, Y \in B_{10 \epsilon}^{n}$.

(vi) $\left|P_{h}(v)-\bar{P}_{h}(v)\right|<1000 n^{3} \tau \epsilon^{2}$ for all unit vectors $v \in T\left(B_{10 \epsilon}^{n}\right)$.

Note that the path $f:[0,1] \rightarrow N$ of (ii) maps into $B_{10 \epsilon}^{n}$ (cf. (iii), and recall that length $(f)=\epsilon$ ); so we may apply (vi) to $f$ to help us verify that $f$ 
satisfies Property (i). Let $L_{0}$ and $L_{1}$ denote the leafs of $\mathcal{G}$ which contain $f(0)$ and $f(1)$ respectively; and let $V_{0}$ and $V_{1}$ denote the planes in $\mathbf{R}^{n}$ which are tangent to $L_{0}$ and $L_{1}$ at $f(0)$ and $f(1)$ respectively. One deduces from 2.6.2 (as applied to $L_{0}$ near $f(0)$, and to $L_{1}$ near $f(1)$ ), and from Properties (iii) and (vi) in the preceding paragraph, that the following holds for sufficiently small $\epsilon>0$ :

(vii) $\bar{d}\left(X, V_{i} \cap B_{10 \epsilon}^{n}\right)<(\alpha / \beta) 10^{3} \epsilon^{2}$ and $\bar{d}\left(Y, L_{i} \cap B_{10 \epsilon}^{n}\right)<(\alpha / \beta) 10^{3} \epsilon^{2}$

for $i=0,1$ and for any $X \in L_{i} \cap B_{10 \epsilon}^{n}$ and any $Y \in V_{i} \cap B_{10 \epsilon}^{n}$. On the other hand, we may deduce from 2.6(i), and from the fact that length $(f)=\epsilon$, that there is a positive number $t \in(0,2 \epsilon)$ such that:

(viii) $d\left(X, L_{0} \cap B_{10 \epsilon}^{n}\right)=t$

for all $X \in L_{1} \cap B_{9 \epsilon}^{n}$. Now we deduce from Properties (vii) and (viii) above, and from Property $(\mathrm{v})$ in the preceding paragraph, that:

(ix) $t-(\alpha / \beta) 10^{4} \epsilon^{2}<\bar{d}\left(Y, V_{0} \cap B_{10 \epsilon}^{n}\right)<t+(\alpha / \beta) 10^{4} \epsilon^{2}$

for all $Y \in V_{1} \cap B_{8 \epsilon}^{n}$. It follows immediately from Property (ix) above, that:

(x) $\bar{\Theta}\left(V_{1}, \bar{P}_{f}\left(V_{0}\right)\right)<(\alpha / \beta) 10^{5} \epsilon$,

where $\bar{\Theta}($,$) denotes the Euclidean angular distance. Finally, since V_{i}=$ $T \mathcal{G}_{f(i)}$ for $i=0,1$, and length $(f)=\epsilon$, we may deduce Property (i) above from Properties (iii) and (x) above.

Proof of Lemma 2.7. Let $L \in \mathcal{G}$ denote the leaf which contains $x$. Towards verifying 6.2.7(a) we will first show that there is an open subset $U$ of $L$ which satisfies the following properties. For any $t>0$ let $B(x ; L ; t)$ denote the open ball in $L$ of radius $t$ centered at $x$ (where the distance $d_{L}($,$) on$ $L$ is gotten by restricting $g_{N}$ to $\left.T L\right)$.

2.7.0. (a) $U$ is homeomorphic to $\mathbf{R}^{k}(k=\operatorname{dim} L)$.

(b) $B(x ; L ; 3 B \beta) \subset U \subset B(x ; L ; 5 B \beta)$.

Moreover for any $y, z \in U$ we have that the distances $d_{L}(y, z)$ and $d_{N}(y, z)$ are related by:

2.7.0. (c) $2 d_{L}(y, z) / 3<d_{N}(y, z) \leq d_{L}(y, z)$.

To verify 2.7.0 we refer to results in the appendix to $\S 1$ above, and use the curvature bound for $\mathcal{G}$ given in 2.6, and also use the hypothesis of 2.7 that $\left(N, g_{N}\right)$ has radius of injectivity greater than $10^{4} \tau B \beta$ at $x$. In more detail we let $f_{x}: B_{10 B \beta}^{n} \rightarrow N$ denote the map given in A.1.1 and A.1.2 (where $\epsilon, p, M, B_{\epsilon}^{m}$ are replaced in A.1.1, A.1.2 by $10 \beta, x, N, B_{10 B \beta}^{n}$ with $n=\operatorname{dim} N)$. Set $\hat{\mathcal{G}}=f_{x}^{-1}(\mathcal{G})$; and let $K\left(\hat{\mathcal{G}} ; B_{10 B \beta}^{n}\right)$ denote the curvature for $\hat{\mathcal{G}}$ in $B_{10 B \beta}^{n}$ with respect to the Euclidean metric (cf. 2.6(ii)). The proof of Theorem A.1.4 also works to show the following (cf. A.1.4.1): 
2.7.0.1. $K\left(\hat{\mathcal{G}} ; B_{10 B \beta}^{n}\right)-\mathcal{O}(10 B \beta)<K(\mathcal{G} ; N)<K\left(\hat{\mathcal{G}} ; B_{10 B \beta}^{n}\right)+\mathcal{O}(10 B \beta)$.

Note that 2.6 and 2.7.0.1 together imply that:

2.7.0.2. $K\left(\hat{\mathcal{G}} ; B_{10 B \beta}^{n}\right)<10^{6} \alpha / \beta+\mathcal{O}(10 B \beta)$.

We let $\hat{L}$ denote the leaf of $\hat{\mathcal{G}}$ through the origin; there is no loss of generality in assuming that the subspace $\mathbf{R}^{k} \subset \mathbf{R}^{n}$ spanned by the first $k$ standard directions in $\mathbf{R}^{n}$ is tangent to $\hat{L}$ at the origin. Now the curvature bounds placed on $\hat{L}$ by 2.7.0.2 assures that $\hat{L}$ is the graph of a smooth map $h: V \rightarrow$ $\mathbf{R}^{n-k}$ from an open subset $V \subset \mathbf{R}^{k}$ which satisfies the following properties:

2.7.0.3. (a) $B_{4 B \beta}^{k} \subset V$, where $B_{t}^{k}$ denotes the open ball of radius $t>0$ centered at the origin of $\mathbf{R}^{k}$.

(b) $h(0)=0$ and $D h_{0}=[0]$.

(c) $\left\|D^{2} h_{p}\right\|<\mathcal{O}(\alpha / \beta+\beta)$ for all $p \in V$.

Now we define the subset $U \subset L$ of 2.7 .0 by:

2.7.0.4. $U=f_{x}\left(\operatorname{graph}\left(h \mid B_{4 B \beta}^{k}\right)\right)$.

Since $N$ has injectivity radius greater than $10^{4} \tau B \beta$ at $x$ for $\tau>1$, it follows that $f_{x}$ is a smooth embedding; thus $U$ is homeomorphic to $B_{4 B \beta}^{k}$, which verifies Property 2.7.0(a). Note that Properties 2.7.0(b) and (c) can be deduced from 2.7.0.3, 2.7.0.4, and from the relation between the Euclidean metric on $B_{10 B \beta}^{n}$ and the pulled back metric $g_{i, j}$ (pulled back from $g_{N}$ by $f_{x}$ ) given in A.1.1, provided $\alpha$ and $\beta$ are sufficiently small.

Now we will complete the proof for 2.7 (a). Let $h, h_{g}: \mathbf{R} \rightarrow G$ be as in 2.7(a). Note that it follows from 2.7.0(b) and (c), and from the inequalities

$$
d_{N}(x, h(s)(x))<B \beta \text { and } d_{N}\left(x, h_{g}(s)(x)\right)<B \beta
$$

for all $s \in[0,1]$ given in the hypotheses of 2.7 , that the following property holds:

2.7.1. (a) $h(s)(x), h_{g}(s)(x) \in U$ for all $s \in[0,1]$.

Since $g \in h([0,1]) \cap h_{g}([0,1])$ we may choose numbers $b, c \in[0,1]$ such that:

2.7.1. (b) $h(b)=g$ and $h_{g}(c)=g$.

Now we may deduce from 2.7.1(a) and (b), from the fact that $U$ is simply connected (cf. 2.7.0(a)), and from the fact the the algebraic exponential map exp : $T \hat{G}_{e} \rightarrow \hat{G}$ is a diffeomorphism (where $e \in \hat{G}$ denotes the identity element of the simply connected covering $\hat{G}$ for $G$ ), that:

2.7.1. (c) $h(b s / c)=h_{g}(s)$ 
for all $s \in \mathbf{R}$. Now setting $a=b / c$ in 2.7(a), we see that Property 2.7(a) is a consequence of 2.7.1(c). (If $g$ is the identity element of $\Gamma_{t, x}$, set $a=0$.)

Towards verifying 2.7(b) we let $\pi: \hat{G} \rightarrow G$ denote the simply connected covering group of $G$, and let $\hat{h}_{g}: \mathbf{R} \rightarrow \hat{G}$ denote the lifting of the $h_{g}: \mathbf{R} \rightarrow G$ to a one-parameter subgroup of $\hat{G}$ (for each $g \in \Gamma_{t, x}$ ). By the hypotheses of 2.7 there is for each $g \in \Gamma_{t, x}$ a number $r_{g} \in[0,1]$ such that $h_{g}\left(r_{g}\right)=g$; let $\hat{\Gamma}_{t, x} \subset \hat{G}$ denote the subgroup generated by all the $\left\{\hat{h}_{g}\left(r_{g}\right): g \in \Gamma_{t, x}\right\}$. Note that $\hat{\Gamma}_{t, x}$ is a torsion free discrete subgroup of $\hat{G}$; thus $\hat{\Gamma}_{t, x}$ is a lattice in a closed connected subgroup $\hat{G}_{t, x} \subset \hat{G}$ (cf. [17, p. 31, Prop. 2.5]). We define $G_{t, x}$ to be the image of $\hat{G}_{t, x}$ under the covering projection $\pi: \hat{G} \rightarrow$ $G$. Note that $G_{t, x} \subset G$ is a closed connected subgroup since $\hat{G}_{t, x} \subset \hat{G}$ is a closed connected subgroup, $\hat{\Gamma}_{t, x}$ is co-compact in $\hat{G}_{t, x}$, and $\pi\left(\hat{\Gamma}_{t, x}\right)$ is discrete. We note (since the algebraic exponential map $T \hat{G}_{e} \rightarrow \hat{G}$ is a diffeomorphism) that $\hat{G}_{t, x}$ is the smallest connected Lie subgroup of $\hat{G}$ which contains each of the one-parameter subgroups $\hat{h}_{g}: \mathbf{R} \rightarrow \hat{G}, g \in \Gamma_{t, x}$. Thus $G_{t, x}$ is the smallest connected Lie subgroup of $G$ which contains each of the one-parameter subgroups $h_{g}: \mathbf{R} \rightarrow G, g \in \Gamma_{t, x}$, as claimed in 2.7(b). This completes the verification for $2.7(\mathrm{~b})$.

Towards verifying Property 2.7 (c) we note that there is an action $\hat{G}_{t, x} \times$ $N \rightarrow N$ defined to be the composition

$$
\hat{G}_{t, x} \times N \subset \hat{G} \times N \stackrel{\pi \times 1_{N}}{\longrightarrow} G \times N \longrightarrow N
$$

where $\pi: \hat{G} \rightarrow G$ denotes the covering projection and where $G \times N \rightarrow N$ is the given action. Note that $\hat{G}_{t, x} \times N \rightarrow N$ acts thru isometries of $N$ and has local angle control equal $(\alpha, \beta)$. Now let the sequence of Lie subgroups $e=G_{1} \subset G_{2} \subset G_{3} \subset \cdots \subset G_{l}=\hat{G}_{t, x}$ be defined by $G_{i-1}=\left[G_{l}, G_{i}\right]$. Note that each $G_{i} \times N \rightarrow N$ acts thru isometries and has local angle control equal to $(\alpha, \beta)$; we let $\mathcal{G}_{i}$ denote the foliation of $N$ by the orbits of the action $G_{i} \times N \rightarrow N$ (cf. 2.5). We let $L_{i} \in \mathcal{G}_{i}$ denote the leaf containing $x$; and we let $d_{L_{i}}($,$) denote the distance on L_{i}$ induced by the restriction of $g_{N}$ to $T L_{i}$. Since $G_{i} \times N \rightarrow N$ also has local angle control equal to $(\alpha, \beta)$ it must also have local angle control equal to $\left(\alpha_{i}, \beta_{i}\right)$, where $\left(\alpha_{i}, \beta_{i}\right)=$ $\left(10^{2 l+2} \alpha t / \beta, 10^{2 l+2} t\right)$ (cf. 2.4). Thus, we may (by repeating the proof of 2.7.0 with $G, L, d_{L}(),,(\alpha, \beta)$ replaced by $\left.G_{i}, L_{i}, d_{L_{i}}(),,\left(\alpha_{i}, \beta_{i}\right)\right)$ choose an open subset $U_{i} \subset L_{i}$ such that the following properties hold. [Note that the hypothesis of 2.7 that $N$ has radius of injectivity greater than $10^{4} \tau B \beta$ assures us that $f_{x}: B_{10 B \beta_{i}}^{n} \rightarrow N$ is an imbedding, provided that $\tau>10^{2 l}$; this is an important part of the proof of 2.7 .0 as applied in the present context.]

2.7.2. (a) $U_{i}$ is homeomorphic to a Euclidean space. 
(b) $B\left(x ; L_{i} ; 3 B \beta_{i}\right) \subset U_{i} \subset B\left(x ; L_{i} ; 5 B \beta_{i}\right)$, where $B\left(x ; L_{i} ; s\right)$ denotes the open ball of radius $s>0$ (with respect to $d_{L_{i}}($,$) ) centered at x$ in $L_{i}$.

(c) $2 d_{L_{i}}(y, z) / 3<d_{N}(y, z) \leq d_{L_{i}}(y, z)$ for all $y, z \in U_{i}$.

We define subsets $H_{i} \subset G_{i}$ as follows: $H_{l}=\left\{\hat{h}_{g}\left(r_{g}\right): g \in \Gamma_{t, x}\right\} ; H_{i-1}$ denotes the set of all commutators $a b a^{-1} b^{-1}$ with $a \in H_{l}$ and $b \in H_{i}$. Now define subsets $\Gamma_{i} \subset G_{i}$ by

$$
\Gamma_{i}=\bigcup_{j=1}^{i} H_{j} .
$$

Let $\bar{\Gamma}_{i}$ denote the subgroup of $G_{i}$ generated by $\Gamma_{i}$. Note that $\bar{\Gamma}_{l}=\hat{\Gamma}_{t, x}$ and that $G_{l}=\hat{G}_{t, x}$; since $\hat{\Gamma}_{t, x}$ is a lattice in $\hat{G}_{t, x}$ (see the verification of $2.7(\mathrm{~b})$ above), we have that $\Gamma_{l}$ is a lattice in $G_{l}$. Thus it follows that for each $i=1,2, \ldots, l$ we have:

2.7.2. (d) $\bar{\Gamma}_{i}$ is a lattice for the simply connected nilpotent Lie group $G_{i}$, and $\Gamma_{i}$ is a generating set of $\bar{\Gamma}_{i}$. Moreover $\widetilde{\Gamma}_{i}$ [defined by $\widetilde{\Gamma}_{i}=\bar{\Gamma}_{l} \cap G_{i}$ ] is a lattice in $G_{i}$ which contains $\bar{\Gamma}_{i}$ as a subgroup of finite index; and $\widetilde{\Gamma}_{i} / \widetilde{\Gamma}_{i-1}$ is a lattice in the abelian Lie group $G_{i} / G_{i-1}$.

Note that it follows from the hypothesis of 2.7 that

$$
d_{N}\left(x, h_{g}(s)(x)\right)<B t
$$

for all $g \in \Gamma_{t, x}$ and all $s \in[0,1]$; so in particular we have

$$
d_{N}\left(x, \hat{h}_{g}\left(r_{g}\right)(x)\right)<B t
$$

for all $g \in \Gamma_{t, x}$. We deduce from these last two inequalities, and from the definition of $\left(\alpha_{i}, \beta_{i}\right)$ and $\Gamma_{i}$ given above, and from 2.7.2(a), (b) and (c), that:

2.7.2. (e) $d_{G_{i}}(x, h(x))<B \beta_{i}$ for all $i=1,2, \ldots, l$ and all $h \in \Gamma_{i}$,

where $d_{G_{i}}($,$) is the distance function induced on G_{i}$ by the pull back of $g_{N}$ under the composite map $G_{i}=G_{i} \times\{x\} \subset G_{i} \times N \rightarrow N$; for future reference we denote this pull back of $g_{N}$ by $g_{G_{i}}$. Note that $g_{G_{i}}$ is a left invariant Riemannian metric on the Lie group $G_{i}$.

Now we can use 2.7.2 to verify 2.7(c). We will use an induction argument, and 2.7.2, to verify that the following holds for all $i=1,2, \ldots, l$ :

2.7.3. diameter $\left(G_{i} / \bar{\Gamma}_{i}\right)<\tau_{i} \beta_{i}$,

where $\tau_{i}>1$ is a number which depends only on $i, \operatorname{dim} G_{i}$ and $B$, and where the diameter is computed with respect to the distance on $G_{i} / \bar{\Gamma}_{i}$ induced by $g_{G_{i}}$. Note that Property 2.7(c) is implied by 2.7.3 (with $i=l$ in 2.7.3), 
provided $\tau$ of $2.7(\mathrm{c})$ satisfies

$$
\tau \geq 10^{2 l+2} \tau_{l}
$$

Towards verifying 2.7.3 we first note that if $G_{i}$ is abelian then 2.7.3 follows immediately from 2.7.2(d) and (e) with $\tau_{i}=B \operatorname{dim} G_{i}$. In particular 2.7.3 is satisfied for $i=2$ with $\tau_{2}=B \operatorname{dim} G_{2}$, which is the beginning of our induction argument. Suppose now that 2.7.3 holds for all $i \leq r$. Note that $G_{r+1} / G_{r}$ is an abelian Lie group equipped with a left invariant Riemannian metric $g_{r+1}$ uniquely determined (from $g_{G_{r+1}}$ ) by the requirement that the quotient map $G_{r+1} \rightarrow G_{r+1} / G_{r}$ be a Riemannian submersion. Thus it follows immediately from 2.7.2(d) and (e) (with $i=r+1$ in 2.7.2(d) and (e)), that

$$
\operatorname{diameter}\left(\left(G_{r+1} / G_{r}\right) /\left(\bar{\Gamma}_{r+1} / \widetilde{\Gamma}_{r}\right)\right)<B \beta_{r+1} \operatorname{dim}\left(G_{r+1} / G_{r}\right) .
$$

Now 2.7.3 (for $i=r+1$ ) follows from this last inequality, and from 2.7.3 (for $i=r$ ), and from the equality $\beta_{r}=\beta_{r+1}$, provided we define $\tau_{r+1}$ by $\tau_{r+1}=\tau_{r}+2 B \operatorname{dim}\left(G_{r+1} / G_{r}\right)$. This completes the verification of 2.7(c).

Now we will complete the proof for 2.7 by verifying $2.7(\mathrm{~d})$. Towards this end we first note that for any $g \in G_{t, x}$ as in $2.7(\mathrm{~d})$ the following property holds:

2.7.4. $d_{L_{t, x}}(x, g(x))<3 t / 2$,

where $d_{L_{t, x}}($,$) is the distance induced by the restricted Riemannian metric$ $g_{N} \mid T\left(L_{t, x}\right)$.

To verify 2.7.4 we will assume it is not true and derive a contradiction. Recall that

$$
d_{N}(x, g(x))<t
$$

is a hypothesis of $2.7(\mathrm{~d})$. Recall also that for $L_{i}$ of 2.7 .2 we have that $L_{i}=L_{t, x}$ when $i=l$. Thus if 2.7.4 doesn't hold then we deduce from the preceding inequality, and from 2.7.2(b) and (c), that:

2.7.5. (a) $d_{L_{t, x}}(x, g(x)) \geq 3 B \beta_{l}$.

The number $\beta_{l}$ of 2.7 .2 was defined to be $10^{2 l+2} t$; however 2.7.2(a)-(c) can also be verified as before if $\left(\alpha_{l}, \beta_{l}\right)$ is chosen to be

$$
\left(\alpha_{l}, \beta_{l}\right)=(100 \tau \alpha t / \beta, 100 \tau t),
$$

where $\tau>1$ comes from 2.7(c) and (d). (Note that the hypothesis of 2.7, that $N$ has radius of injectivity greater than $10^{4} \tau B \beta$, at $x$, implies that the map $f_{x}: B_{10 B \beta_{1}}^{n} \rightarrow N$ is an embedding for $\beta_{1}=100 \tau t$; this is an important part of the verification of 2.7.2(a)-(c) for our present choice of $\left(\alpha_{l}, \beta_{l}\right)$.) This last equality, and Properties 2.7.5(a) and 2.7.2(c), together imply: 
2.7.5. (b) $d_{L_{t, x}}(x, g(x))>100 \tau B t$.

Using 2.7(c) we may choose $\bar{g} \in \bar{\Gamma}_{t, x}$ such that:

2.7.5. (c) $d_{L_{t, x}}(g(x), \bar{g}(x))<\tau t$.

Now combining 2.7.5(b) and (c), and recalling that $B>1$ (cf. A.2.3), we deduce that:

2.7.5. (d) $d_{L_{t, x}}(x, \bar{g}(x))>90 \tau B t$.

Now this last inequality leads to a contradiction (which will complete the verification of 2.7.4) as follows: Since $d_{N}(x, g(x))<t$, and $\tau>1$, it follows from 2.7.5(c) that $d_{N}(x, \bar{g}(x))<2 \tau t$, and so by definition of $\Gamma_{2 \tau t, x}$ we have that $\bar{g} \in \Gamma_{2 \tau t, x}$. Then the hypothesis of $2.7(\mathrm{~d})$ (that $G_{t, x}=G_{2 \tau t, x}$ ), together with 2.7(b), assures us that Image $\left(h_{\bar{g}}\right) \subset G_{t, x}$ (where $h_{\bar{g}}$ comes from the hypothesis of 2.7). Thus the equation $f_{\bar{g}}(s)=h_{\bar{g}}(s) x$ defines a path $f_{\bar{g}}$ : $[0,1] \rightarrow L_{t, x}$ which starts at $x$ and contains $\bar{g} x$ and which (by the hypothesis of 2.7 applied to $h_{\bar{g}}$ ) satisfies:

2.7.5. (e) $d_{N}\left(x, f_{\bar{g}}(s)\right)<2 \tau B t$

for all $s \in[0,1]$. Now 2.7.5(e) and 2.7.2(b) and (c), together with the continuity of $f_{\bar{g}}$ imply:

2.7.5. (f) $d_{L_{t, x}}\left(x, f_{\bar{g}}(s)\right)<4 \tau B t$

for all $s \in[0,1]$. [When applying 2.7.2(c) to verify 2.7.5(f) we set $i=l$ and $\beta_{l}=100 \tau t$ in 2.7(b) and (c), as in the derivation of 2.7.5(b) above.] Since $\bar{g} x \in$ image $\left(f_{\bar{g}}\right)$, we see that 2.7.5(f) contradicts 2.7.5(d). This contradiction completes the verification of 2.7.4.

In our verification of $2.7(\mathrm{~d})$ we need also consider the composite map

$$
T G_{e} \stackrel{\exp }{\longrightarrow} G \stackrel{h}{\longrightarrow} N,
$$

where $T G_{e}$ is the tangent space for $G$ at the identity $e \in G$, exp $: T G_{e} \rightarrow$ $G$ is the algebraic exponential map, and $h: G \rightarrow N$ is given by $h(g)=$ $g(x)$. Note that $h \circ \exp$ is an immersion (cf. 2.5). TG $G_{e}$ is equipped with an inner product gotten by pulling $g_{N} \mid T\left(L_{t, x}\right)_{x}$ back along the linear map $D(\exp \circ h): T G_{e} \rightarrow T\left(L_{t, x}\right)_{x}$. For each unit vector $v \in T G_{e}$ we have the one-parameter subgroup $\rho_{v}: \mathbf{R} \rightarrow G$ defined by $\rho_{v}(s)=\exp (s v)$. Define the action $\gamma_{v}: \mathbf{R} \times N \rightarrow N$ by $\gamma_{v}(s, y)=\rho_{v}(s)(y)$. In order to complete the proof for $2.7(\mathrm{~d})$ we shall need that the $\gamma_{v}$ satisfy the following property:

2.7.6. $\left|d_{N}\left(x, \gamma_{v}(s, x)\right)-s\right|<\mathcal{O}(\alpha) s$ for all $s \in[-4 \beta, 4 \beta]$.

Towards verifying 2.7.6 we first note that each action $\gamma_{v}: \mathbf{R} \times N \rightarrow N$ has local angle control equal $(\alpha, \beta)$, because the action $G \times N \rightarrow N$ has local 
angle control equal $(\alpha, \beta)$. So (by 2.5 applied to $\gamma_{v}$ ) the orbits of the action $\gamma_{v}: \mathbf{R} \times N \rightarrow N$ are the leaves of a smooth foliation $\mathcal{G}_{v}$ of $N$; and we may apply 2.6 to each action $\gamma_{v}: \mathbf{R} \times N \rightarrow N$ to conclude that:

2.7.7. (a) $K\left(\mathcal{G}_{v} ; N\right)<10^{6} \alpha / \beta$.

Next we consider again the map $f_{x}: B_{10 \beta}^{n} \rightarrow N$ given in A.1.1 and A.1.2 (where $\epsilon, p, M, B_{\epsilon}^{m}$ are replaced in A.1.1, A.1.2 by $10 \beta, x, N, B_{10 \beta}^{n}$ with $n=$ $\operatorname{dim} N)$. Set $\hat{\mathcal{G}}_{v}=f_{x}^{-1}\left(\mathcal{G}_{v}\right)$; and let $K\left(\hat{\mathcal{G}}_{v} ; B_{10 \beta}^{n}\right)$ denote the curvature for $\hat{\mathcal{G}}_{v}$ in $B_{10 \beta}^{n}$ with respect to the Euclidean metric (cf. 2.6(ii)). The proof of Theorem A.1.4 also works to show the following (cf. A.1.4.1):

2.7.7. (b) $K\left(\hat{\mathcal{G}}_{v} ; B_{10 \beta}^{n}\right)-\mathcal{O}(10 \beta)<K\left(\mathcal{G}_{v} ; N\right)<K\left(\hat{\mathcal{G}}_{v} ; B_{10 \beta}^{n}\right)+\mathcal{O}(10 \beta)$.

Now by combining 2.7.7(a) and (b) we conclude that:

2.7.7. (c) $K\left(\hat{\mathcal{G}}_{v} ; B_{10 \beta}^{n}\right)<10^{6} \alpha / \beta+\mathcal{O}(10 \beta)$.

Let $g_{i, j} d x_{i} d x_{j}$ denote the Riemannian metric on $B_{10 \beta}^{n}$ gotten by pulling back $g_{N}$ along $f_{x}: B_{10 \beta}^{n} \rightarrow N$; let $\hat{d}($,$) denote the distance on B_{10 \beta}^{n}$ induced by $g_{i, j} d x_{i} d x_{j}$, and let $d($,$) denote the Euclidean distance on B_{10 \beta}^{n}$. Let $\hat{L}_{v}$ denote the (connected) leaf of $\hat{\mathcal{G}}_{v}$ which contains the origin. Denote by $\hat{d}_{v}($,$) the distance induced on \hat{L}_{v}$ by the restriction to $T \hat{L}_{v}$ of $g_{i, j} d x_{i} d x_{j}$; and denote by $d_{v}($,$) the distance on \hat{L}_{v}$ induced by the restriction to $T \hat{L}_{v}$ of the Euclidean structure $\delta_{j}^{i} d x_{i} d x_{j}$. Note that we can deduce from 2.7.7(c) that:

2.7.7. (d) $\left|d(0, y)-d_{v}(0, y)\right|<\mathcal{O}(\alpha) d_{v}(0, y)$,

for all $y \in \hat{L}_{v}$, provided $\alpha, \beta$ are sufficiently small (as assumed in the hypothesis of 2.7). [In more detail we could use 2.7.7(c) (in place of 2.7.0.2 above) to verify that $\hat{\mathcal{G}}_{v}$ satisfies Properties 2.7.0.3(a) and (b) for $k=1$; where in 2.7.0.3 $h: V \rightarrow \mathbf{R}^{n-1}$ is a smooth map from an open subset $V \subset \mathbf{R}$ such that $\hat{L}_{v}$ is equal to the graph of $h$. Then we could deduce 2.7.7(d) from this version of 2.7.0.3.] Next we note it follows from the relation between the metrics $g_{i, j} d x_{i} d x_{j}$ and $\delta_{j}^{i} d x_{i} d x_{j}$ given in Theorem A.1.1, and also given in A.1.3.3.1, A.1.3.4.1, that:

2.7.7. (e) $|d(0, y)-\hat{d}(0, y)|+\left|d_{v}(0, y)-\hat{d}_{v}(0, y)\right|<\mathcal{O}\left(d_{v}(0, y)\right) d_{v}(0, y)$

for all $y \in \hat{L}_{v}$, provided $\beta$ is sufficiently small. (Note that $d(0, y)=\hat{d}(0, y)$.) Now by combining 2.7.7(d) and (e) we can deduce that:

2.7.7. (f) $\left|\hat{d}(0, y)-\hat{d}_{v}(0, y)\right|<\mathcal{O}(\alpha) \hat{d}_{v}(0, y)$ 
for all $y \in \hat{L}_{v}$ with $\hat{d}(0, y) \leq 4 \beta$, provided $\alpha$ and $\beta$ are sufficiently small and $\beta<\alpha$ (as hypothesized in 2.7). Finally we recall that $f_{x}: B_{10 \beta}^{n} \rightarrow N$ is an embedding, since one of the hypotheses of 6.2 .7 is that $N$ has injectivity radius greater than $10^{4} \tau B \beta$ at $x$ with $\tau>1$ and $B>1$. Thus, the desired inequality 2.7.6 is equivalent to the inequality of $2.7 .7(\mathrm{f})$.

Now we can complete the proof of 2.7 (d) by applying 2.7.4, 2.7.6, and 2.7.2(a)-(c) (with $i=l$ in 2.7.2(a)-(c)). For any $s>0$ let $B_{e}(s)$ denote the closed ball of radius $s$ centered at the origin of $T G_{e}$, and let $L_{l}$ and $B\left(x ; L_{l}, s\right)$ and $\beta_{l}>0$ be as in 2.7.2(a), (b) and (c) (recall $\beta_{l}=10^{2 l+2} t$ ). Recall that exp : $T G_{e} \rightarrow G$ denotes the algebraic exponential map and $h: G \rightarrow N$ is given by $h(g)=g(x)$. Note that $2.7(\mathrm{~d})$ may be deduced directly from Property 2.7.4 and the following property. (Also recall that $G_{l}$ of 2.7.2(a)-(c) is equal $\hat{G}_{t, x}$.)

\subsection{8. $B\left(x ; L_{l} ; 3 t / 2\right) \subseteq h \circ \exp \left(B_{e}(2 t) \cap T G_{t, x}\right)$.}

Towards verifying 2.7.8 we note that it follows from 2.7.2(a) and (b), and from 2.7.6, and from the equality $G_{l}=\hat{G}_{t, x}$, that $h \circ \exp \left(B_{e}(2 t) \cap T G_{t, x}\right) \subset$ $U_{l}$ and $h \circ \exp : B_{e}(2 t) \rightarrow U_{l}$ is a smooth embedding, provided $\alpha>0$ is sufficiently small. Now since $B_{e}(2 t) \cap T G_{t, x}$ is a closed ball, and $U_{l}$ is homeomorphic to Euclidean space (cf. 2.7.2(a)), and $\operatorname{dim}\left(B_{e}(2 t) \cap T G_{t, x}\right)=$ $\operatorname{dim} U_{l}$, it follows that the complement in $U_{l}$ of the sphere $h \circ \exp \left(\partial\left(B_{e}(2 t) \cap\right.\right.$ $\left.T G_{t, x}\right)$ ) consists of two open connected sets $U_{l}^{+}$and $U_{l}^{-}$, with

$$
U_{l}^{+}=h \circ \exp \left(\left(B_{e}(2 t) \cap T G_{t, x}\right)-\partial\left(B_{e}(2 t) \cap T G_{t, x}\right)\right) .
$$

It follows from 2.7.6 that

$$
B\left(x ; L_{l} ; 3 t / 2\right) \cap h \circ \exp \left(\partial\left(B_{e}(2 t) \cap T G_{t, x}\right)\right)=\phi
$$

provided $\alpha>0$ is sufficiently small; so the connected set $B\left(x ; L_{l} ; 3 t / 2\right)$ must be contained in one of $U_{l}^{+}$or $U_{l}^{-}$. Since both $U_{l}^{+}$and $B\left(x ; L_{l} ; 3 t / 2\right)$ contain the point $x$, we have that

$$
B\left(x ; L_{l} ; 3 t / 2\right) \subset U_{l}^{+} .
$$

This completes the verification of 2.7.8.

Proof of Lemma 2.8. The proof is carried out in the following six steps:

Step I. In this step we let $G$ be a connected nilpotent Lie group with Lie algebra $\mathfrak{g} ; G$ is equipped with a left invariant Riemannian metric. We will say that $G$ has $(\alpha, \beta)$-angular control when the left multiplication group action $G \times G \rightarrow G$ has local angle control equal $(\alpha, \beta)$.

The purpose of this step is to verify the following claim. The notation $\hat{u}$ will denote the left invariant vector field on $G$ determined by a vector $u \in \mathfrak{g}$. 
Claim 2.8.1. Assume $\alpha<\pi$. Then, $G$ has $(\alpha, \beta)$-angular control iff for all $u, v \in \mathfrak{g}$ we have

$$
\left|D_{\hat{v}} \hat{u}\right| \leq(\alpha / \beta)|u||v|
$$

Towards verifying 2.8.1 we re-look at the Proof of Lemma 2.3 in the special case when $N=G, G \times G \rightarrow G$ is the group multiplication, and $x=e$. And observe that

$$
d / d t\left(A_{-v}\right)_{t=0}(u)=D_{\hat{v}} \hat{u}(e)
$$

for each pair $u, v \in \mathfrak{g}$ where $|v|=1$. But, $d / d t\left(A_{-v}\right)_{t=0}$ is the blocked sum of the zero matrix of a certain size and $2 \times 2$ matrices of the form

$$
\left[\begin{array}{cc}
0 & -\theta \\
\theta & 0
\end{array}\right]
$$

where $0 \leq \theta \leq \alpha / \beta$. Now 2.8 .1 is easily seen to follow from these observations.

Step II. In this step we let $G$ be a connected nilpotent Lie group with Lie algebra $\mathfrak{g} ; G$ is equipped with a left invariant Riemannian metric. The purpose of this step is to verify the following claim. In this claim we set $k=\operatorname{dim} G$.

Claim 2.8.2. If $G$ has $(\alpha, \beta)$-angular control with $\alpha<\pi$, then for any pair $x, y \in \mathfrak{g}$ we have

$$
|[x, y]| \leq\left(2 k^{2} \alpha / \beta\right)|x||y|
$$

Since $\mathfrak{g}$ is nilpotent, we can fix an orthonormal basis $x_{1}, x_{2}, \ldots, x_{k}$ for $\mathfrak{g}$ (with $k=\operatorname{dim} \mathfrak{g}$ ) such that

$$
\left[x_{i}, x_{j}\right] \in \operatorname{Span}\left\{x_{0}, x_{1}, \ldots, x_{i-1}\right\}
$$

for every pair of indices $i, j \geq 1$. (We define $x_{0}=0$.) Recall the following standard formula (cf. [2, Prop. 7.7.1]). Let $X, Y$, and $Z$ be left invariant vector fields on $G$, then

$$
\left(D_{X} Y\right) \cdot Z=1 / 2\{-[X, Y] \cdot Z+[Y, Z] \cdot X+[X, Z] \cdot Y\}
$$

where the "dot" indicates the inner product of vector fields. Applying this formula where $X \in \operatorname{Span}\left\{\hat{x}_{i}\right\}, Y \in \operatorname{span}\left\{\hat{x}_{j}\right\}$, and $Z=[X, Y]$, yields

$$
\left(D_{X} Y\right) \cdot[X, Y]=-1 / 2[X, Y] \cdot[X, Y] .
$$

And combining this equation with Claim 2.8.1, yields:

2.8.2.1. $|[X, Y]| \leq(2 \alpha / \beta)|X||Y|$

provided $G$ has $(\alpha, \beta)$-angular control and $\alpha<\pi$. 
Now we will deduce Claim 2.8.2 from 2.8.2.1 as follows: Set $\hat{x}=\sum_{i=1}^{k} X_{i}$ and $\hat{y}=\sum_{i=1}^{k} Y_{i}$ where $X_{i}, Y_{i} \in \operatorname{Span}\left(\hat{x}_{i}\right)$. Then, by 2.8.2.1, we have

$$
|[x, y]| \leq \sum_{i, j=1}^{k}\left|\left[X_{i}, Y_{j}\right]\right| \leq \sum_{i, j=1}^{k}(2 \alpha / \beta)\left|X_{i}\right|\left|Y_{j}\right|
$$

Since $\left\{x_{1}, x_{2}, \ldots, x_{k}\right\}$ is an orthonormal basis we also have that

$$
\sum_{i, j=1}^{k}(2 \alpha / \beta)\left|X_{i}\right|\left|Y_{j}\right|=(2 \alpha / \beta)\left(\sum_{i=1}^{k}\left|X_{i}\right|\right)\left(\sum_{i=1}^{k}\left|Y_{i}\right|\right) \leq(2 \alpha / \beta)(k|x|)(k|y|) .
$$

Combining these last two sets of inequalities yields 2.8.2.

Step III. We now let $G$ be a simply connected nilpotent Lie group with Lie algebra $\mathfrak{g} ; G$ is equipped with a left invariant Riemannian metric. The purpose of this step is to verify the following claim:

Claim 2.8.3. Let $\mathfrak{h}$ be an ideal in $\mathfrak{g}$ and let $H$ be the corresponding (closed and normal) subgroup of $G$. If $G$ has $(\alpha, \beta)$-angular control, then $G / H$ also has $(\alpha, \beta)$-angular control provided we equip $G / H$ with the (unique) left invariant Riemannian metric which makes the quotient homomorphism $q: G \rightarrow G / H$ a Riemannian submersion.

Note that $H$ is closed because $G$ is simply connected.

Let $\mathbf{m}=\mathfrak{h}^{\perp}$; i.e., $\mathbf{m}$ is the orthogonal complement to $\mathfrak{h}$ in $\mathfrak{g}$. Identify $\mathbf{m}$ with $\mathfrak{g} / \mathfrak{h}$ via the quotient map $\mathfrak{g} \rightarrow \mathfrak{g} / \mathfrak{h}$. And, for each $u \in \mathbf{m}$, let $\check{u}$ denote the left invariant vector field on $G / H$ determined by $u$. Denote the covariant derivative operators for $G$ and $G / H$ by $\bar{D}$ and $D$ respectively. Since the quotient map $q: G \rightarrow G / H$ is a Riemannian submersion, we may apply [16, p. 212, Lemma 45$]$ to get that

$$
\left|\bar{D}_{\hat{v}} \hat{u}\right| \geq\left|D_{\check{v}} \check{u}\right|
$$

for all $u, v \in \mathbf{m}$. From this inequality, and also from Claim 2.8.1, we conclude that $G / H$ has $(\alpha, \beta)$-angular control. (Note that we may assume that $\alpha<\pi$ in 2.8.1 since Claim 2.8.3 is otherwise trivially true.) This completes the verification for Claim 2.8.3.

Step IV. In this step we let $G$ be a simply connected nilpotent Lie group with Lie algebra $\mathfrak{g} ; G$ is equipped with a left invariant Riemannian metric. Let $\gamma_{i}: \mathbf{R} \rightarrow G$ be the one-parameter subgroup of $G$ such that $\dot{\gamma}(0)=x_{i}$ (where the $\left\{x_{i}\right\}$ come from Step II). Define a map $f: \mathbf{R}^{k} \rightarrow G$ by

$$
f\left(t_{1}, t_{2}, \ldots, t_{k}\right)=\gamma_{1}\left(t_{1}\right) \gamma_{2}\left(t_{2}\right) \ldots \gamma_{k}\left(t_{k}\right) .
$$


Then it is a classical result for simply connected nilpotent Lie groups that $f$ is a diffeomorphism. Thus, for each $g \in G$, we may define coordinates $\left\{g_{i}\right\}$ for $g$ by

$$
\left(g_{1}, g_{2}, \ldots, g_{k}\right)=f^{-1}(g) .
$$

The purpose of this step is to verify the following claim:

Claim 2.8.4. Suppose that $G$ has $(\alpha, \beta)$-angular control where $\alpha$ is sufficiently small. (How small is sufficient depends only on $k=\operatorname{dim} G$.) The following inequality holds for each $g \in G$ with $d_{G}(g, e) \leq \beta$ :

$$
\left|g_{1}\right| \leq 2 d_{G}(g, e) .
$$

To verify this claim we proceed by induction on $k=\operatorname{dim} G$. (We may assume that $k>1$ since 2.8 .4 is clearly true when $k=1$.) Consider the function $\psi: G \rightarrow \mathbf{R}$ defined by

$$
\psi(h)=h_{1}
$$

for $h \in G$, and let the exact 1-form $\omega$ be defined by

$$
\omega=d \psi \text {. }
$$

Let $\gamma:[0,1] \rightarrow G$ be a constant speed smooth path such that:

2.8.4.1. (a) $\gamma(0)=e$ and $\gamma(1)=g$,

(b) length $(\gamma)=d_{G}(g, e)$.

Since $g_{1}=\int_{0}^{1} \omega(\dot{\gamma}(t)) d t$, we also have that:

2.8.4.1. (c) $\left|g_{1}\right| \leq \int_{0}^{1}|\omega(\dot{\gamma}(t))| d t$.

Now, by 2.8.4.1, in order to complete the verification for Claim 2.8.4 it will suffice to verify the following property:

2.8.4.2. There exists a positive real number $C_{k}$ such that the following is true if $\alpha \leq C_{k}$. For each $h \in G$ with $d_{G}(h, e) \leq \beta$ and for each $v \in T G_{h}$ we have that $|\omega(v)| \leq 2|v|$.

To verify 2.8.4.2 we let $H$ denote the image of the one-parameter subgroup $\gamma_{1}$ and set $\mathfrak{h}=\operatorname{span}\left\{x_{1}\right\}$. Then, $G / H$ is a simply connected nilpotent Lie group whose Lie algebra is $\mathfrak{g} / \mathfrak{h}$ and the quotient homomorphism $q: G \rightarrow$ $G / H$ does not increase distances. (We equip $G / H$ with the left invariant Riemannian metric so that $q$ is a Riemannian submersion.) Also, $G / H$ has $(\alpha, \beta)$-angular control because of Claim 2.8.3. For each index $i \geq 0$ let $\bar{x}_{i}$ 
denote the coset $x_{i+1}+\mathfrak{h}$. Then $\bar{x}_{1}, \bar{x}_{2}, \ldots, \bar{x}_{k-1}$ is an orthonormal basis for $\mathfrak{g} / \mathfrak{h}$ such that

$$
\left[\bar{x}_{i}, \bar{x}_{j}\right] \in \operatorname{Span}\left\{\bar{x}_{0}, \bar{x}_{1}, \ldots, \bar{x}_{i-1}\right\}
$$

for all $i, j \geq 1$. Let $\bar{f}: \mathbf{R}^{k-1} \rightarrow G / H$ be the diffeomorphism corresponding to this basis (defined analogously to $f: \mathbf{R}^{k} \rightarrow G$ above). And notice that

$$
h_{2}=[q(h)]_{1}
$$

where $h$ comes from 2.8.4.2 and where $[q(h)]_{1}$ is the first coordinate of $\bar{f}^{-1}(q(h))$. Hence our inductive assumption yields that

$$
\left|h_{2}\right| \leq 2 d_{G / H}(q(h), e) \leq 2 d_{G}(h, e) .
$$

We see continuing in this way that:

2.8.4.3. (a) $\left|h_{i}\right| \leq 2 d_{G}(h, e) \leq 2 \beta$

for all $i \geq 2$. Associate to $h$ a finite sequence of group elements $h(1), h(2)$, $\ldots, h(k) \in G$ defined by

$$
h(i)=f\left(0,0, \ldots, 0, h_{i+1}, h_{i+2}, \ldots, h_{k}\right) .
$$

And define a finite sequence of vectors $y_{1}, y_{2}, \ldots, y_{k} \in \mathfrak{g}$ by:

2.8.4.3. (b) $y_{i}=\operatorname{Ad}\left(h(i)^{-1}\right)\left(x_{i}\right)$

where Ad $: G \rightarrow G L(\mathfrak{g})$ is the adjoint representation. Then note that:

2.8.4.3 (c) $\left\{\hat{y}_{2}(h), \hat{y}_{3}(h), \ldots, \hat{y}_{k}(h)\right\}$ is a basis for $\operatorname{Ker}\left(\omega_{h}\right)$, and $y_{1}=x_{1}$,

where $\omega_{h}: T G_{h} \rightarrow \mathbf{R}$ is the restriction of the 1 -form $\omega$ to the tangent space to $G$ at $h$. (Recall $\hat{x}$ is the left invariant vector field determined by each $x \in \mathcal{G}$.) A continuity argument, together with 2.8.4.3(c), shows that there exists a positive number $\bar{C}_{k}$ such that if:

2.8.4.3. (d) $\left|y_{i}-x_{i}\right| \leq \bar{C}_{k}$

for all $i \geq 2$, then $|\omega(v)| \leq 2|v|$ (as desired in 2.8.4.2). We proceed to show that there exists a positive number $C_{k}$ such that if $\alpha \leq C_{k}$, then 2.8.4.3(d) holds for all indices $i$. (This will complete the verification of 2.8.4.2, and hence also of Claim 2.8.4.)

We note that by the definitions of $h(i)$ and of $f: \mathbf{R}^{k} \rightarrow G$ we have that:

2.8.4.4. (a) $h(i)=\gamma_{i+1}\left(h_{i+1}\right) \gamma_{i+2}\left(h_{i+2}\right) \ldots \gamma_{k}\left(h_{k}\right)$.

Substituting 2.8.4.4(a) into 2.8.4.3(b) yields:

2.8.4.4. (b) $y_{i}=\operatorname{Ad}\left(\gamma_{k}\left(-h_{k}\right)\right) \ldots \operatorname{Ad}\left(\gamma_{i+1}\left(-h_{i+1}\right)\right)\left(x_{i}\right)$.

Note now that, for all indices $j$, we have: 
2.8.4.4. (c) $\operatorname{Ad}\left(\gamma_{j}\left(-h_{j}\right)\right)=e^{-h_{j} \operatorname{ad}\left(x_{j}\right)}$

where $\operatorname{ad}\left(x_{j}\right): \mathfrak{g} \rightarrow \mathfrak{g}$ is defined by $\operatorname{ad}\left(x_{j}\right)(u)=\left[x_{j}, u\right]$ for all $u \in \mathfrak{g}$. (The equation in 2.8.4.4(c) follows from [12, formula (5), p. 118], and from the fact that $\gamma_{j}\left(-h_{j}\right)=\operatorname{Exp}\left(-h_{j} x_{j}\right)$ where $\operatorname{Exp}: \mathfrak{g} \rightarrow G$ is the algebraic exponential map.) Now, by combining 2.8.4.4(c) with Claim 2.8.2 and 2.8.4.3(a) and $\left|x_{i}\right|=1$, we obtain:

2.8.4.4. (d) $\left|\operatorname{Ad}\left(\gamma_{j}\left(-h_{j}\right)\right)(u)-u\right| \leq|u|\left(e^{4 k^{2} \alpha}-1\right)$

for all indices $j$ and every $u \in \mathfrak{g}$. In particular, we have that:

2.8.4.4. (e) $\left|\operatorname{Ad}\left(\gamma_{j}\left(-h_{j}\right)\right)(u)\right| \leq|u| e^{4 k^{2} \alpha}$.

It now follows from 2.8.4.4(b), (d) and (e), and from the fact that $\left|x_{i}\right|=1$ for all $i$, that:

2.8.4.4. (f) $\left|y_{i}-x_{i}\right|<e^{4 k^{3} \alpha}-1$

for all indices $i$. This last inequality shows that the positive number $C_{k}$ (posited above) clearly exists since $e^{x}$ is continuous and $e^{0}=1$.

This completes the verification of Claim 2.8.4.

Step V. In this step we let $G$ be a simply connected nilpotent Lie group with Lie algebra $\mathfrak{g} ; G$ is equipped with a left invariant Riemannian metric. Recall that the algebraic exponential map Exp $: \mathfrak{g} \rightarrow G$ is defined by $\operatorname{Exp}(v)=\gamma_{v}(1)$ where $\gamma_{v}$ is the one parameter subgroup of $G$ such that $\dot{\gamma}_{v}(0)=v$. It is a classical result for simply connected nilpotent Lie groups that Exp is a diffeomorphism. The purpose of this step is to verify the following claim. In this claim we set $k=\operatorname{dim} G$ and let $e \in G$ denote the identity element.

Claim 2.8.5. Suppose that $G$ has $(\alpha, \beta)$-angular control where $\alpha$ is sufficiently small. (How small is sufficient depends only on $\operatorname{dim} G$.) Then, for each $g \in G$ such that $d_{G}(g, e) \leq \beta$, we have that

$$
\left|\operatorname{Exp}^{-1}(g)\right| \leq 4^{k} d_{G}(g, e) .
$$

One easily observes that the following statement is a consequence of the proof given above for Lemma 2.3:

2.8.5.1. For every $\lambda \geq 1,(\alpha, \beta)$-angular control implies $(\lambda \alpha, \lambda \beta)$-angular control.

Now to verify Claim 2.8.5 we proceed by induction on $k=\operatorname{dim} G$. (We may assume $k>1$ since 2.8.5 is clearly true when $k=1$.) Let $H$ denote the image of the one-parameter subgroup $\gamma_{1}$; it is a closed subgroup contained in the center of $G$. Hence, $G / H$ is also a simply connected nilpotent Lie 
group whose Lie algebra is $\mathfrak{g} / \mathfrak{h}$ where $\mathfrak{h}=\operatorname{Span}\left\{x_{1}\right\}$ is the Lie algebra of $H$. Put the (unique) left invariant Riemannian metric on $G / H$ which makes the quotient homorphism $q: G \rightarrow G / H$ a Riemannian submersion. Note that $G / H$ also has $(\alpha, \beta)$-angular control (by Claim 2.8.3) and 2.8.5 is valid for $G / H$ since $\operatorname{dim}(G / H)=k-1$. Set

$$
\operatorname{Exp}^{-1}(g)=s x_{1}+u
$$

where $u \in \operatorname{Span}\left\{x_{2}, x_{3}, \ldots, x_{k}\right\}$, and let

$$
b=\operatorname{Exp}(u) \text { and } c=\operatorname{Exp}\left(s x_{1}\right)=\gamma_{1}(s) .
$$

Note that:

2.8.5.2. (a) $g=c b$

since $c \in \operatorname{Center}(G)$. Also let $\bar{g}$ denote $q(g)$. Then:

2.8.5.2. (b) $d_{G / H}(\bar{g}, e) \leq d_{G}(g, e) \leq \beta$

since $q: G \rightarrow G / H$ does not increase distances. Also note that $\operatorname{Exp}_{G / H}(u+$ $\mathfrak{h})=\bar{g}$ where $\operatorname{Exp}_{G / H}: \mathfrak{g} / \mathfrak{h} \rightarrow G / H$ denotes the algebraic exponential map for $G / H$. Applying 2.8.5 to $\bar{g}$, and using 2.8.5.2(b), we obtain that:

2.8.5.2. (c) $|u| \leq 4^{k-1} d_{G}(g, e)$.

Of course, this last inequality implies directly that:

2.8.5.2. (d) $d_{G}(b, e) \leq 4^{k-1} d_{G}(g, e)$.

Now, 2.8.5.2(a), and the fact that $d_{G}($, ) is left invariant, imply that:

2.8.5.2. (e) $d_{G}(c, e) \leq d_{G}(g, e)+d_{G}(e, b)$.

Combining 2.8.5.2(d) and (e) yields:

2.8.5.2. (f) $d_{G}(c, e) \leq\left(4^{k-1}+1\right) d_{G}(g, e)$.

Since $\gamma_{1}(s)=c$, we also have that:

2.8.5.2. (g) $c_{1}=s$

where $c_{1}$ is the first coordinate of $f^{-1}(c)$.

Now let $C_{k}$ be the positive real number such that the conclusion of Claim 2.8.4 is true (in dimension $k$ ) provided $\alpha \leq C_{k}$, and let $\bar{C}_{k-1}$ be the corresponding number such that the conclusion of Claim 2.8.5 is true (in dimension $k-1)$ provided $\alpha \leq \bar{C}_{k-1}$. Set

$$
\bar{C}_{k}=\min \left\{\bar{C}_{k-1},\left(4^{k-1}+1\right)^{-1} C_{k}\right\} .
$$


In particular, we assume that $G$ has $(\alpha, \beta)$-angular control where $\alpha \leq \bar{C}_{k}$. And $G$ also has $\left(C_{k}, \bar{\beta}\right)$-angular control where

$$
\bar{\beta}=\left(4^{k-1}+1\right) \beta
$$

because of 2.8.5.1. Now note that $d_{G}(c, e) \leq \bar{\beta}$ because of 2.8.5.2(f) and our assumption that $d_{G}(g, e)<\beta$. We can consequently apply 2.8 .4 to $c$ and, using again 2.8.5.2(f), conclude that:

2.8.5.3. (a) $\left|c_{1}\right| \leq 2 d_{G}(c, e) \leq 2\left(4^{k-1}+1\right) d_{G}(g, e)$.

But the triangle inequality, and 2.8.5.2(g), and $\left|x_{1}\right|=1$, together yield that:

2.8.5.3. (b) $\left|\operatorname{Exp}^{-1}(g)\right|=\left|s x_{1}+u\right| \leq\left|c_{1}\right|+|u|$.

Now, by combining the inequalities of 2.8.5.2(c) and 2.8.5.3(a) and (b) we get that

$$
\left|\operatorname{Exp}^{-1}(g)\right| \leq 4^{k} d_{G}(g, e)
$$

as required in 2.8.5.

This completes the proof for Claim 2.8.5.

Step VI. In this step we shall complete the proof for Lemma 2.8. We assume $s>0$ since the conclusion of 2.8 is trivially true when $s=0$. Let $\rho: \widetilde{G} \rightarrow G$ denote the universal covering group of $G$ and put the induced left invariant Riemannian metric on $\widetilde{G}$. Clearly, $\widetilde{G}$ also has $(\alpha, \beta)$-angular control. Let the one parameter subgroup $\widetilde{\phi}_{1}: \mathbf{R} \rightarrow \widetilde{G}$ be the unique lift of $\phi_{1}$. Furthermore, let $\mu:[0,1] \rightarrow G$ be a smooth path such that:

2.8.6. (a) length $(\mu)<t$,

(b) $\mu(0)=e$ and $\mu(1)=\phi_{1}(s)$.

And, let $\widetilde{\mu}:[0,1] \rightarrow \widetilde{G}$ be the unique lift of $\mu$ such that $\widetilde{\mu}(0)=e$. Set $g=\widetilde{\mu}(1)$ and observe that $d_{\widetilde{G}}(g, e) \leq \beta$ (cf. 2.8.6(a) and recall $t<\beta$ ). Thus we may apply Claim 2.8.5 to $\widetilde{G}$ to get a (unique) one-parameter subgroup $\gamma:[0, s] \rightarrow \widetilde{G}$ such that:

2.8.6. (c) $\gamma(s)=g$,

$$
\text { (d) }|\dot{\gamma}(0)| \leq s^{-1} 4^{k} d_{\widetilde{G}}(g, e) \text {. }
$$

The following useful inequality is a direct consequence of 2.8.6(d) and of the fact that $\gamma$ has constant speed:

2.8.6. (e) $d_{\widetilde{G}}(\gamma(u), e) \leq(u / s) 4^{k} t$ for all $u \in[0, s]$.

We denote by $C \subset G$ and $\widetilde{C} \subset \widetilde{G}$ the centers of $G$ and $\widetilde{G}$, respectively. Note that $\widetilde{C}=p^{-1}(C)$; consequently, $G / C=\widetilde{G} / \widetilde{C}$ is a simply connected 
nilpotent Lie group. Let $q: G \rightarrow G / C$ denote the quotient homomorphism. Then, the two one-parameter subgroups $q \circ \rho \circ \gamma$ and $q \circ \phi_{1}$ are the same because the algebraic exponential map for $G / C$ is a diffeomorphism and because of the equation

$$
q \circ \rho \circ \gamma(s)=q \circ \phi_{1}(s)
$$

Consequently, there is a one-parameter subgroup $\psi: \mathbf{R} \rightarrow \widetilde{C}$ such that $\widetilde{\phi}_{1}$ factors as

$$
\widetilde{\phi}_{1}(u)=\psi(u) \gamma(u)
$$

for all $u \in \mathbf{R}$. Now, set

$$
\phi_{2}=\rho \circ \psi
$$

Then, $\phi_{1}$ factors as

$$
\phi_{1}(u)=\left(\phi_{2}(u)\right)(\rho \circ \gamma(u))
$$

for all $u \in \mathbf{R}$. Evaluating this factorization at $u=s$ yields that $\phi_{2}(s)=e$ (since $\rho \circ \gamma(s)=\phi_{1}(s)$ ). Thus Statement (b) of 2.8 is verified.

Since the metric on $G$ is left invariant, the last factorization also yields that

$$
d_{G}\left(\phi_{1}(u), \phi_{2}(u)\right)=d_{G}(\rho \circ \gamma(u), e)
$$

for all $u \in[0, s]$. And observe that

$$
d_{G}(\rho \circ \gamma(u), e) \leq d_{\widetilde{G}}(\gamma(u), e)
$$

because $\rho: \widetilde{G} \rightarrow G$ does not increase distances. Combining these last two facts with 2.8.6(e) yields that statement (c) of Lemma 2.8 is true.

This completes the proof for Lemma 2.8 .

\section{Proof of Theorem 0.3 .}

It is recommended that the reader consult Appendix 2 before reading this section.

Let $\eta, \epsilon, \delta_{i}$ be as in 1.3. Let $\epsilon, \delta$ be as in Appendix 2 (cf. A.2.1-A.2.3); so as not to confuse the $\epsilon$ of 1.3 with the $\epsilon$ of Appendix 2, we shall henceforth denote the latter by $\bar{\epsilon}$. Recall that $E$ denotes the total space of the frame bundle $\rho: E \rightarrow \hat{V}$ defined in Appendix 2. We choose $\eta, \epsilon, \delta_{i}$ of 1.3 and $\bar{\epsilon}, \delta$ of Appendix 2 as follows, where $m=\operatorname{dim} M$ :

3.0. (a) $\eta=(m+4)^{8}$.

(b) $\delta_{i+1} \ll \epsilon \delta_{i}^{\operatorname{dim} E+2}$.

(c) $\delta_{1} \ll \delta<\bar{\epsilon} \ll \epsilon<1$. 
(To satisfy inequalities (b) and (c) first choose $\delta$ and $\bar{\epsilon}$ and then choose the $\left.\delta_{1}, \delta_{2}, \ldots\right)$

We shall prove Theorem 0.3 when $n=0$ in 0.3 : The proof for $n>0$ is essentially the same. With this in mind it will prove convenient to introduce the following notation for some of the first $(m+4)^{8}$ of the $\left\{\delta_{i}\right\}$. [Note that the following numbers do not exhaust all of the first $(m+4)^{8}$ of the $\left\{\delta_{i}\right\}$.]

3.0. (d) For any integers $i, j, k, l \in\left\{1,2, \ldots,(m+4)^{2}-1\right\}$ we set

$$
\begin{aligned}
t_{i} & =\delta_{i(m+4)^{6}} \\
t_{i, j} & =\delta_{i(m+4)^{6}+j(m+4)^{4},} \\
t_{i, j, k} & =\delta_{i(m+4)^{6}+j(m+4)^{4}+k(m+4)^{2},} \\
t_{i, j, k, l} & =\delta_{i(m+4)^{6}+j(m+4)^{4}+k(m+4)^{2}+l} .
\end{aligned}
$$

For given $p \in M$ we use Theorems A.2.2 and A.2.3 to choose a smooth group action $H \times \hat{V} \rightarrow \hat{V}$ which satisfies A.2.1(a)-(c) such that the lifted action $H \times E \rightarrow E$ satisfies A.2.3(a) and (b). Let $H_{e} \subset H$ denote the identity component, and set $\Lambda_{e}=\Lambda \cap H_{e}$ (see A.2.1 for $\Lambda \subset H$ ). The remainder of this proof is carried out in Subsections 3.1-3.5 below.

3.1. The groups $G_{t_{i}, x}, C_{i, j, x}$, and $\Lambda_{i, j, k, x}$. In this subsection we identify the $G \times N \rightarrow N$ and $g_{N}$ and $\Gamma \subset G$ of $\S 2$ with $H_{e} \times E \rightarrow E$ and $g_{E}^{\prime}$ and $\Lambda_{e} \subset H_{e}$ of Appendix 2. We choose $x$ of 2.7 to lie in the pre-image of $p \in V$ under the composition of projection maps $E \stackrel{\rho}{\longrightarrow} \hat{V} \stackrel{\pi}{\longrightarrow} V$. Then we have that the conclusions of Lemmas 2.4, 2.6 and 2.7 hold for $\beta=\delta$ and $\alpha=\lambda \delta$ for any $\delta>0$ (cf. 2.2 and A.2.5).

The closed connected Lie subgroups $G_{t_{i}, x} \subset G$ are as given in 2.7. We note that $G_{t_{i+1}, x} \subset G_{t_{i}, x}$ for all $1 \leq i<(m+4)^{2}-1$. Thus a dimension argument gives us the following. (Recall that $\operatorname{dim} M=m$ and $\operatorname{dim} E \leq m^{2}+m$; so $\operatorname{dim} G \leq m^{2}+m$ (cf. A.2.3).)

3.1.1. For some $1 \leq i<(m+4)^{2}-1$ we have that

$$
G_{t_{i}, x}=G_{t_{i+1}, x} .
$$

For each $1 \leq j \leq(m+4)^{2}-1$, and for $i$ as in 3.1.1, we choose a subgroup $C_{i, j, x} \subset G_{t_{i}, x}$ which is maximal in $G_{t_{i}, x}$ with respect to the following properties:

3.1.2. (a) $d_{\hat{V}}^{\prime}(\rho(x), h \rho(x)) \leq t_{i, j}$ for each $h \in C_{i, j, x}$.

(b) $C_{i, j, x}$ is compact.

The existence of such a maximal subgroup of $G_{t_{i}, x}$ can be seen as follows: Since $G_{t_{i}, x}$ is a connected nilpotent Lie group (cf. 2.7) it must contain a single maximal compact subgroup $C \subset G_{t_{i}, x}$. Now if $C_{1} \subset C_{2} \subset C_{3} \subset \ldots$ is a 
sequence of compact subgroups satisfying 3.1.2(a) and (b) with $C_{l} \neq C_{l+1}$ for all $l$, then $D=$ closure $\left(\bigcup_{l=1}^{\infty} C_{l}\right)$ is a subgroup of $C$ which satisfies Properties 3.1.2(a) and (b) and also satisfies $\operatorname{dim}\left(C_{1}\right)<\operatorname{dim}(D)$. Thus to construct $C_{i, j, x}$ we can first choose a compact subgroup $C_{1}$ of maximal dimension that satisfies 3.1.2(a) and (b); then by the preceding remarks $C_{n}$ (one of the groups in the sequence $\left.C_{1} \subset C_{2} \subset C_{3} \subset \ldots\right)$ may be chosen to be $C_{i, j, x}$.

Note that these compact subgroups can be chosen to satisfy $C_{i, j+1, x} \subset$ $C_{i, j, x}$ for all $1 \leq j<(m+4)^{2}-1$. We denote by $F_{i, j, x} \subset \hat{V}$ the connected component of the fixed point set for $C_{i, j, x} \times \hat{V} \rightarrow \hat{V}$ which is a distance less than $2 t_{i, j}$ from $\rho(x)$ (cf. Remark 3.1.3.1 below to see that $\left.F_{i, j, x} \neq \phi\right) . F_{i, j, x}$ is a totally geodesic submanifold of $\hat{V}$. It follows from 3.1.2 that each $F_{i, j, x}$ is a nonempty closed subset of $\hat{V}$; and that the equality $C_{i, j, x}=C_{i, j+1, x}$ would be implied by the equality $F_{i, j, x}=F_{i, j+1, x}$. On the other hand, it follows from the inclusion $C_{i, j+1, x} \subset C_{i, j, x}$ that $F_{i, j, x} \subset F_{i, j+1, x}$. Thus a dimension argument (over the dimension of the fixed point sets) can be used to verify the following:

3.1.3. For some $1 \leq j<(m+4)^{2}-1$ we have that

$$
C_{i, j, x}=C_{i, j+1, x} \text {. }
$$

Remark 3.1.3.1. We show in this remark that $F_{i, j, x} \neq \phi$; i.e., there is a fixed point $Y \in \hat{V}$ for the action $C_{i, j, x} \times \hat{V} \rightarrow \hat{V}$ with $d_{\hat{V}}^{\prime}(\rho(x), Y)<2 t_{i, j}$. Since $\hat{V}, g_{V}^{\prime}$ is $A^{\prime}$-regular with radius of injectivity at $\rho(x)$ much greater than $t_{i, j}$ (cf. 3.0 and Appendix 2), we may choose an imbedding $f_{p}: B_{\bar{\epsilon}}^{m} \rightarrow \hat{V}$ as in A.1.1 and A.1.2 with $p=\rho(x)$ and $\bar{\epsilon} \gg t_{i, j}$. The action of $C_{i, j, x} \times \hat{V} \rightarrow \hat{V}$ pulls back along $f_{p}$ to give a smooth action $C_{i, j, x} \times B_{\bar{\epsilon} / 2}^{m} \rightarrow B_{\bar{\epsilon}}^{m}$ defined by the requirement that $f_{p}(h q)=h f_{p}(q)$ for all $h \in C_{i, j, x}$ and all $q \in B_{\bar{\epsilon} / 2}^{m}$ (cf. 3.1.2(a)). In light of A.1.1(a) and (b), Property 3.1.2(a) implies that the pulled back action satisfies:

(i) $|h 0|<(3 / 2) t_{i, j}$

for all $h \in C_{i, j, x}$, where $h 0$ indicates the action of $h$ on the origin $0 \in B_{\bar{\epsilon} / 2}^{m}$. Now if $\hat{V}$ were a Riemannian flat space $\left(g_{i, j}=\delta_{j}^{i}\right.$ everywhere in A.1.1(a) and (b)) we could obtain a fixed point element for the action $C_{i, j, x} \times B_{\bar{\epsilon} / 2}^{m} \rightarrow B_{\bar{\epsilon}}^{m}$ by taking the geometric average:

(ii) $X=\left(\int_{C_{i, j, x}} h 0\right) / \operatorname{Vol}\left(C_{i, j, x}\right)$

of all the values in (i). Note that (i) would assure that $X$ of (ii) satisfies $|X|<(3 / 2) t_{i, j}$; so setting $Y=f_{p}(X)$ we get the desired fixed point for the action $C_{i, j, x} \times \hat{V} \rightarrow \hat{V}$. In the general situation (when $\hat{V}$ is not flat) we 
obtain a fixed point set for the action $C_{i, j, x} \times B_{\bar{\epsilon} / 2}^{m} \rightarrow B_{\bar{\epsilon}}^{m}$ as the limit of points $X_{n}, n=1,2,3, \ldots$, where:

(iii) $X_{1}=X$; and $X_{n+1}=\left(\int_{C_{i, j, x}} h X_{n}\right) / \operatorname{Vol}\left(C_{i, j, x}\right)$ for $n \geq 1$.

Note that (i) and (iii), together with Properties A.1.1(a) and (b) and A.1.3.3.1 in Appendix 1, imply that:

(iv) $X_{\infty}=\operatorname{limit}_{n \rightarrow \infty} X_{n}$

exists and satisfies:

(v) $\left|X_{\infty}\right|<(7 / 4) t_{i, j}$ and $h X_{\infty}=X_{\infty}$

for all $h \in C_{i, j, x}$. Setting $Y=f_{p}\left(X_{\infty}\right)$ we get the desired fixed point for the action $C_{i, j, x} \times \hat{V} \rightarrow \hat{V}$. [Note that (v) above, and Properties A.1.1(a) and (b) in Appendix 1, together imply that $d_{\hat{V}}^{\prime}(\rho(x), Y)<2 t_{i, j}$.]

For each $1 \leq k \leq(m+4)^{2}-1$, and for $i$ and $j$ as in 3.1.1 and 3.1.3, we let $\Lambda_{i, j, k, x} \subset \Lambda$ denote the subgroup generated by all $g \in \Lambda$ which satisfy:

3.1.4. $d_{\hat{V}}^{\prime}(\rho(x), g \rho(x))<t_{i, j, k}$.

We use the remainder of this subsection to verify the following claim:

Claim 3.1.5. The following relations exist between the groups $G_{t_{i}, x}, C_{i, j, x}$, $\Lambda_{i, j, k, x}$, for $i$ and $j$ as in 3.1.1 and 3.1.3 and each $1 \leq k<(m+4)^{2}-1$ :

(a) $g\left(G_{t_{i}, x}\right) g^{-1}=G_{t_{i}, x}$ for each $g \in \Lambda_{i, j, k, x}$.

(b) $g\left(C_{i, j, x}\right) g^{-1}=C_{i, j, x}$ for each $g \in \Lambda_{i, j, k, x} \cup G_{t_{i}, x}$.

(c) Set $\Lambda_{i, j, k, x}^{\prime}=\Lambda_{i, j, k, x} \cap G_{t_{i}, x}$. Then $\Lambda_{i, j, k, x}^{\prime}$ is a normal subgroup of $\Lambda_{i, j, k, x}$ of index $\ll t_{i}^{-\operatorname{dim} E}$.

(d) $\Lambda_{i, j, 1, x}^{\prime}=\Lambda_{i, j, k, x}^{\prime}$.

\section{Verification of Claim 3.1.5.}

It will suffice to verify 3.1.5(a) for any $g \in \Lambda_{i, j, k, x}$ satisfying 3.1.4. Note that any $g^{\prime} \in \Gamma_{t_{i+1}, x}$ satisfies:

3.1.6. (a) $d_{E}^{\prime}\left(x, h_{g^{\prime}}(s) x\right)<B t_{i+1}$

for all $s \in[0,1]$, where $h_{g^{\prime}}$ is the one-parameter subgroup hypothesized in 2.7 for $t=t_{i+1}$, and where $B$ comes from A.2.3 and 2.7. And, since $\rho: E \rightarrow \hat{V}$ is a Riemannian submersion, 3.1.6(a) immediately implies:

3.1.6. (b) $d_{\hat{V}}^{\prime}\left(\rho(x), h_{g^{\prime}}(s) \rho(x)\right)<B t_{i+1}$

for all $s \in[0,1]$. Combining 3.1.4 with 3.1.6(b) we get:

3.1.6. (c) $d_{\hat{V}}^{\prime}\left(\rho(x), g h_{g^{\prime}}(s) g^{-1} \rho(x)\right)<2 t_{i, j, k}+B t_{i+1}$ 
for all $s \in[0,1]$. Finally, by combining 3.1.6(a) and (c) with the inequality $2 t_{i, j, k}+B t_{i+1} \ll t_{i}$ (cf. 3.0), we may deduce that:

3.1.7. (a) $d_{E}^{\prime}\left(x, g\left(h_{g^{\prime}}(s)\right) g^{-1}(x)\right) \ll t_{i}$

for all $s \in[0,1]$. Recall that $g^{\prime}=h_{g^{\prime}}\left(r_{g^{\prime}}\right)$ for some $r_{g^{\prime}} \in[0,1]$; so when $s=r_{g^{\prime}}$ in $3.1 .7($ a) we get:

3.1.7. (b) $d_{E}^{\prime}\left(x, g g^{\prime} g^{-1}(x)\right) \ll t_{i}$.

We also have that:

3.1.7. (c) $g g^{\prime} g^{-1} \in \Gamma$.

To see this first note that $g g^{\prime} g^{-1} \in \Lambda$; and, since $g^{\prime} \in H_{e}$ and $g H_{e} g^{-1}=H_{e}$, we also have that $g g^{\prime} g^{-1} \in H_{e}$. Now 3.1.7(c) follows from these remarks and the equality $\Gamma=\Lambda \cap H_{e}$. An immediate consequence of Properties 3.1.7(b) and (c), and of the definition of $\Gamma_{t, x}$ given just prior to 2.7 above, is that:

3.1.8. (a) $g g^{\prime} g^{-1} \in \Gamma_{t_{i}, x}$.

Thus, there is the one-parameter subgroup $h_{g g^{\prime} g^{-1}}: \mathbf{R} \rightarrow G_{t_{i}, x}$ hypothesized in 2.7. Consider also the one-parameter subgroup $h: \mathbf{R} \rightarrow H_{e}$ defined by $h(s)=g h_{g^{\prime}}(s) g^{-1}$. We note that 3.1.7(a) assures that 2.7(a) may be applied to conclude that $h$ is just a reparameterization for $h_{g g^{\prime} g^{-1}}$; in particular we have that:

3.1.8. (b) $g h_{g^{\prime}}(s) g^{-1} \in G_{t_{i}, x}$ for all $s$.

Since the one-parameter subgroups $h_{g^{\prime}}: \mathbf{R} \rightarrow G_{t_{i+1}, x}, g^{\prime} \in \Gamma_{t_{i+1}, x}$, generate $G_{t_{i+1}, x}$ (cf. 2.7(b)), it follows from 3.1.8(b) that:

3.1.8. (c) $g G_{t_{i+1}, x} g^{-1} \subset G_{t_{i}, x}$.

Now Property 3.1.5(a) is a consequence of Properties 3.1.1 and 3.1.8(c).

In verifying Claim 3.1.5(b), since $C_{i, j, x}$ is in the center of $G_{t_{i}, x}$, we only need to consider the case where $g \in \Lambda_{i, j, k, x}$. It also suffices to consider $g$ which satisfies 3.1.4 since $\Lambda_{i, j, k, x}$ is generated by such elements. It follows from 3.1.2 and from 3.1.4 that:

3.1.9. $d_{\hat{V}}^{\prime}\left(\rho(x), g h g^{-1} \rho(x)\right)<2 t_{i, j, k}+t_{i, j+1}$, for all $h \in C_{i, j+1, x}$.

Let $C \subset G$ denote the minimal compact subgroup which contains both $C_{i, j+1, x}$ and $g\left(C_{i, j+1, x}\right) g^{-1}$. Since $C$ is in the center of $G$ it follows that any element in $C$ can be written as a product $g_{1} g_{2}$ of two elements in $C_{i, j+1, x} \cup$ $g\left(C_{i, j+1, x}\right) g^{-1}$. This last fact, together with 3.1.2(a) (as applied to $C_{i, j+1, x}$ ) and 3.1.9, implies that: 
3.1.10. (a) $d_{\hat{V}}^{\prime}(\rho(x), h \rho(x))<\lambda\left(2 t_{i, j, k}+t_{i, j+1}\right)$ for all $h \in C$.

It follows from 3.0 that

$$
\lambda\left(2 t_{i, j, k}+t_{i, j+1}\right) \ll t_{i, j} .
$$

Combining this last inequality with 3.1.10(a) we get that:

3.1.10. (b) $d_{\hat{V}}^{\prime}(\rho(x), h \rho(x))<t_{i, j}$ for all $h \in C$.

If 3.1.5(b) doesn't hold, then $C_{i, j+1, x} \subset C$ but $C_{i, j+1, x} \neq C$ (cf. 3.1.3). These last properties, together with 3.1.2, 3.1.3 and 3.1.10(b), contradict the maximality property for $C_{i, j, x}$. This completes the verification of Claim 3.1.5(b).

Now we verify Claim 3.1.5(c). It follows directly from 3.1.5(a) that $\Lambda_{i, j, k, x}^{\prime}$ is a normal subgroup of $\Lambda_{i, j, k, x}$. To estimate its index choose a covering of the fiber $\rho^{-1}(\rho(x))$ by a finite number of open subsets $X_{1}, \ldots, X_{q} \subset \rho^{-1}(\rho(x))$ which satisfy the following properties:

\subsubsection{1.}

(a) Each $X_{s}$ is an open ball in $\rho^{-1}(\rho(x))$ of radius $t_{i} / 8$. (Here the radius is measured with respect to the restriction of $g_{E}^{\prime}$ to $\rho^{-1}(\rho(x))$.)

(b) $q \ll t_{i}^{-\operatorname{dim} E}$.

Let $B\left(\rho(x), t_{i} / 8\right) \subset \hat{V}$ denote the open ball in $\hat{V}$ of radius $t_{i} / 8$ centered at $\rho(x) \in \hat{V}$, and define an open covering $Y_{1}, \ldots, Y_{q}$ for $\rho^{-1}\left(B\left(\rho(x), t_{i} / 8\right)\right)$ as follows:

\subsubsection{1.}

(c) Each $Y_{s}$ is gotten by parallel translating the set $X_{s}$ along all the geodesics in $B\left(\rho(x), t_{i} / 8\right)$ which start at $\rho(x)$.

If for $h_{1}, h_{2} \in \Lambda_{i, j, k, x}$ we have that $h_{1}(x), h_{2}(x) \in Y_{s}$ (for some $s \in\{1,2, \ldots$, $q\}$ ), it follows from 3.1.11(a) and (c) that $d_{E}^{\prime}\left(x, h_{2}^{-1} h_{1}(x)\right)<t_{i}$. It follows from this last inequality, and from A.2.3 and 3.0, that $h_{2}^{-1} h_{1} \in \Gamma=\Lambda \cap H_{e}$. Thus $h_{2}^{-1} h_{1} \in \Gamma_{t_{i}, x}$ (cf. the definition of $\Gamma_{t_{i}, x}$ which precedes 2.7 , and recall that $\Gamma=\Lambda \cap H_{e}$ ); so $h_{2}^{-1} h_{1} \in G_{t_{i}, x}$ by 2.7 (b). It follows that there at most $q$ distinct left cosets of $\Lambda_{i, j, k, x}^{\prime}$ in $\Lambda_{i, j, k, x}$. This completes the verification of Claim 3.1.5(c).

Finally we will verify $3.1 .5(d)$. First we note that:

3.1.12. $\Gamma_{t_{i, j, k}, x} \subset \Lambda_{i, j, k, x}^{\prime}$.

To see this we recall that $\Gamma=\Lambda \cap H_{e}$, and thus (by the definition of $\Gamma_{t_{i, j, k}, x}$ preceding 2.7 and the definition of $\Lambda_{i, j, k, x}$ in 3.1.4) it follows that $\Gamma_{t_{i, j, k}, x} \subset$ $\Lambda_{i, j, k, x}$. We conclude from 2.7(b) and 3.0 that $\Gamma_{t_{i, j, k}, x} \subset G_{t_{i}, x}$. Now these 
last two inclusions, together with the definition given for $\Lambda_{i, j, k, x}^{\prime}$ in 3.1.5(c), imply 3.1.12. Next we note that it follows from 2.7(c) (with $t=t_{i+1}$ in 2.7) and 3.1.1 that:

3.1.13. diameter $\left(L_{t_{i, j, k}, x} / \bar{\Gamma}_{t_{i, j, k}, x}\right)<\tau t_{i+1}$

for all $1 \leq k \leq(m+4)^{2}-1$. From the definition given for $\Lambda_{i, j, k, x}^{\prime}$ in 3.1.5(c), and from 3.0 and 3.1.1, it follows that $\Lambda_{i, j, k, x}^{\prime}$ acts on the space $L_{t_{i, j, k}, x}$; and thus there is the orbit space $L_{t_{i, j, k}, x} / \Lambda_{i, j, k, x}^{\prime}$. Now combining 3.1 .12 and 3.1.13 we conclude (even when $k=(m+4)^{2}-1$ ) that:

3.1.14. diameter $\left(L_{t_{i, j, k}, x} / \Lambda_{i, j, k, x}^{\prime}\right)<\tau t_{i+1}$.

Setting $k=1$ in 3.1 .14 we see that:

3.1.15. diameter $\left(L_{t_{i, j, 1}, x} / \Lambda_{i, j, 1, x}^{\prime}\right)<\tau t_{i, j, k}$, for $1 \leq k \leq(m+4)^{2}-1$.

It follows immediately from 3.1.15 that there is a generating set $S \subset \Lambda_{i, j, 1, x}^{\prime}$ for the group $\Lambda_{i, j, 1, x}^{\prime}$ such that

$$
d_{E}^{\prime}(x, g x)<2 \tau t_{i, j, k}
$$

for all $g \in S$; and, since $\rho: E \rightarrow \hat{V}$ is a Riemannian submersion, this last inequality implies

$$
d_{\hat{V}}^{\prime}(\rho(x), g \rho(x))<2 \tau t_{i, j, k}
$$

for all $g \in S$. It follows from 3.0 that

$$
2 \tau t_{i, j, k} \ll t_{i, j, k-1} .
$$

Combining these last two inequalities with the definition given for $\Lambda_{i, j, k-1, x}$ in 3.1.4, and with the definitions of $\Lambda_{i, j, 1, x}^{\prime}$ and $\Lambda_{i, j, k-1, x}^{\prime}$ given in 3.1.5(c), we see that:

3.1.16. $\Lambda_{i, j, 1, x}^{\prime} \subset \Lambda_{k, j, k-1, x}^{\prime}$ for $1<k \leq(m+4)^{2}-1$.

On the other hand it is a clear consequence of 3.1.4 and 3.1.5(c), and of the fact that $t_{i, j, k} \leq t_{i, j, 1}$ if $k \geq 1$ (cf. 3.0), that:

3.1.17. $\Lambda_{i, j, k, x}^{\prime} \subset \Lambda_{i, j, 1, x}^{\prime}$ for $1 \leq k \leq(m+4)^{2}-1$.

Now 3.1.16 and 3.1.17 together imply 3.1.5(d).

3.2. The group action $G_{i, j, x} \times F_{i, j, k, y} \rightarrow F_{i, j, k, y}$. Let the positive integers $i, j$ be as in 3.1.1 and 3.1.3. We have referred to the subset $F_{i, j, x} \subset \hat{V}$ in 3.1: It is the connected component of the fixed point set for the action $C_{i, j, x} \times \hat{V} \rightarrow \hat{V}$ which is a distance less than $2 t_{i, j}$ from $\rho(x)$. Note that it follows from 3.1.2, 3.1.3, 3.1.5 that $F_{i, j, x}$ satisfies the following properties: 
3.2.1. (a) $d_{\hat{V}}^{\prime}(\rho(x), y)<2 t_{i, j+1}$, for some $y \in F_{i, j, x}$.

(b) $F_{i, j, x}$ is invariant under the action $G_{t_{i}, x}: \hat{V} \rightarrow \hat{V}$.

(c) $F_{i, j, x}$ is invariant under the action $\Lambda_{i, j, k, x}: \hat{V} \rightarrow \hat{V}$ for all $1 \leq k \leq$ $(m+4)^{2}-1$.

Property 3.2.1(a) follows from 3.1.1-3.1.3. To verify Property 3.2.1(c) it will suffice to show that $g F_{i, j, x}=F_{i, j, x}$ for any $g \in \Lambda_{i, j, k, x}$ which satisfies 3.1.4. Note that by 3.1.5(b) we have that $g F_{i, j, x}$ is a connected component of the fixed point set for $C_{i, j, x} \times \hat{V} \rightarrow \hat{V}$. Using Appendix 2, 3.0, 3.1.4, 3.2.1(a) we have that $y \in F_{i, j, x}$ and $g y \in g F_{i, j, x}$ are a distance apart less than $t_{i, j}$, and that $t_{i, j}$ is much less than the radius of injectivity for $\hat{V}$ at $y$. Thus there is a unique shortest geodesic arc connecting $y$ to $g y$, which must be in the fixed point set for $C_{i, j, x} \times V \rightarrow V$ because $y$ and $g y$ are fixed points. It follows that $F_{i, j, x}=g F_{i, j, x}$. This completes the verification for 3.2.1(c). The verification of 3.2.1(b) is the same as that for 3.2.1(c); note that since $G_{t_{i}, x}$ is connected it has a generating set of elements $g \in G_{t_{i}, x}$ which satisfy Property 3.1.4.

We see from 3.2.1(b) (see also 3.1.5(b)) that there is an action

$$
G_{i, j, x} \times F_{i, j, x} \rightarrow F_{i, j, x}
$$

by the quotient group

$$
G_{i, j, x}=G_{t_{i}, x} / C_{i, j, x}
$$

by $3.2 .1(\mathrm{c})$ we see that there is the action

$$
\Lambda_{i, j, k, x} \times F_{i, j, x} \rightarrow F_{i, j, x}
$$

for all $1 \leq k \leq(m+4)^{2}-1$. For each $1 \leq k \leq(m+4)^{2}-1$, we let

$$
F_{i, j, k, y} \subset F_{i, j, x}
$$

denote the union of all the orbits of the action $G_{i, j, x} \times F_{i, j, x} \rightarrow F_{i, j, x}$ which intersect with $\bigcup g\left(B\left(y ; t_{i, j, k}\right)\right)$ where $B\left(y ; t_{i, j, k}\right)$ denotes the open ball $g \in \Lambda_{i, j, k, x}$

in $F_{i, j, x}$ of radius $t_{i, j, k}$ centered at $y$, and $y \in F_{i, j, x}$ comes from 3.2.1(a). Since $F_{i, j, k, y}$ is the union of some $G_{i, j, x}$-orbits, it is therefore left invariant by the action $G_{i, j, x} \times F_{i, j, x} \rightarrow F_{i, j, x}$. To see that $F_{i, j, k, y}$ is left invariant by the action $\Lambda_{i, j, k, x} \times F_{i, j, x} \rightarrow F_{i, j, x}$ we must appeal to 3.1.5(a). Thus we have the following:

3.2.2. $F_{i, j, k, y}$ is left invariant by each of the actions $G_{i, j, x} \times F_{i, j, x} \rightarrow F_{i, j, x}$ and $\Lambda_{i, j, k, x} \times F_{i, j, x} \rightarrow F_{i, j, x}$.

We use the remainder of this subsection for verifying the following two claims. In both these claims we let $i, j$ be as in 3.1.1, 3.1.3. 
Claim 3.2.3. For any $2 \leq k<(m+4)^{2}-1$ the following hold:

(a) The action $G_{i, j, x} \times F_{i, j, k, y} \rightarrow F_{i, j, k, y}$ has local angle control equal $\left(\epsilon, t_{i, j, 2}\right)$.

(b) $G_{i, j, x} \times F_{i, j, k, y} \rightarrow F_{i, j, k, y}$ is a free action.

(c) Let $\mathcal{G}_{i, j, k, y}$ denote the foliation of $F_{i, j, k, y}$ by the orbits of this action. Then $\mathcal{G}_{i, j, k, y}$ is a strongly Riemannian foliation which satisfies

$$
K\left(\mathcal{G}_{i, j, k, y} ; F_{i, j, k, y}\right)<10^{6} \epsilon t_{i, j, 2}^{-1} .
$$

(See Remarks 2.6(i) and (ii) for terminology and notation.)

For each $t>0$ let $A^{\perp}(y ; t)$ denote the set of all vectors $v \in T\left(F_{i, j, 2, y}\right)_{y}$ which are perpendicular to $\mathcal{G}_{i, j, 2, y}$ and have length $<t$. Note that the exponential map exp : $A^{\perp}(y ; t) \rightarrow \hat{V}$ is a well-defined smooth embedding for all $t \in\left(0, t_{i, j, 2}\right]$ (cf. A.2.1, A.2.2, 3.0); denote its image by $B^{\perp}(y ; t)$.

Claim 3.2.4. For any $4 \leq k<(m+4)^{2}-1$ we have that $B^{\perp}\left(y ; t_{i, j, k-1}\right)$ intersects each leaf of $\mathcal{G}_{i, j, k, y}$ at most once.

\section{Verification of Claim 3.2.3.}

Note that Lemma 2.6 and 3.2.3(a) together imply Property 3.2.3(c). Towards verifying Property 3.2.3(b) we denote by $G_{i, j, x, z}$ the isotropy group for the action $G_{i, j, x} \times F_{i, j, k, y} \rightarrow F_{i, j, k, y}$ at any point $z \in F_{i, j, k, y}$. To verify 3.2.3(b) it will suffice to show that $\left|G_{i, j, x, z}\right|=1$. And, since $G_{i, j, x, g z}=g\left(G_{i, j, x, z}\right) g^{-1}$ for any $g \in G_{i, j, x} \cup \Lambda_{i, j, k, x}$ (cf. 3.1.5(a) and (b)) and

$$
F_{i, j, k, y}=\bigcup_{g_{1} \in G_{i, j, x}, g_{2} \in \Lambda_{i, j, k, x}} g_{1} g_{2} B\left(y ; t_{i, j, k}\right),
$$

it follows that we only have to show that $\left|G_{i, j, x, z}\right|=1$ for $z \in B\left(y ; t_{i, j, k}\right)$. Note that Lemma 2.5 and 3.2.3(a), together with Corollary A.2.4, imply that $\left|G_{i, j, x, z}\right|<\infty$. So the pre-image of $G_{i, j, x, z}$ under the quotient map $G_{t_{i}, x} \rightarrow G_{i, j, x}$ is a finite extension $C_{i, j, x} \subset \bar{C}_{i, j, x}$ of the compact group $C_{i, j, x}$. Note also that $z$ is in the fixed point set for the action $\bar{C}_{i, j, x} \times \hat{V} \rightarrow \hat{V}$, and that $d_{\hat{V}}(\rho(x), z) \ll t_{i, j}$ (use 3.0, 3.2.1(a), and $z \in B\left(y ; t_{i, j, k}\right)$ ). Thus, if $\left|G_{i, j, x, z}\right|>1, \bar{C}_{i, j, x}$ will be a larger compact group than $C_{i, j, x}$ which also satisfies 3.1.2. This would contradict the maximality property for $C_{i, j, x}$. So we must have that $\left|G_{i, j, x, z}\right|=1$ as desired.

We have just argued that Properties 3.2.3(b) and (c) can be deduced from Property 3.2.3(a). Thus to complete the proof of Claim 3.2.3 it will suffice to verify $3.2 .3(\mathrm{a})$. Note also that $F_{i, j, k, y} \subset F_{i, j, 2, y}$ for all $k \geq 2$; so it will suffice to verify 3.2.3(a) for $k=2$. To verify 3.2.3(a) for $k=2$ it will suffice to show that the action $G_{i, j, x} \times F_{i, j, 2, y} \rightarrow F_{i, j, 2, y}$ has local angle control equal $\left(\epsilon, t_{i, j, 2}\right)$ at every $z \in B\left(y, t_{i, j, 2}\right)$ since $g G_{i, j, x} g^{-1}=G_{i, j, x}$ for 
all $g \in G_{i, j, x} \cup \Lambda_{i, j, 2, x}$ by 3.1.5(a) and (b), and

$$
F_{i, j, 2, y}=\bigcup_{g_{1} \in G_{i, j, x}, g_{2} \in \Lambda_{i, j, 2, x}} g_{1} g_{2} B\left(y ; t_{i, j, 2}\right) .
$$

If the desired local angle control does not hold at some $z \in B\left(y, t_{i, j, 2}\right)$ then (by Lemma 2.3) there must exist a unit speed path $f: \mathbf{R} \rightarrow F_{i, j, 2, y}$ and a unit vector $v \in T\left(F_{i, j, 2, y}\right)_{z}$ which satisfy the following properties:

3.2.5. (a) There is a one-parameter subgroup $\phi: \mathbf{R} \rightarrow G_{i, j, x}$ such that $f(t)=\phi(t)(z)$.

(b) $\Theta\left(D g(v), P_{f \mid[0, r]}(v)\right)>\epsilon$, where $g=\phi(r)$ for some $0<r \leq t_{i, j, 2}$.

Choose $\hat{z} \in \rho^{-1}(z)$, and let $\hat{\phi}: \mathbf{R} \rightarrow G_{t_{i}, x}$ denote a one-parameter subgroup such that the composition $\mathbf{R} \stackrel{\hat{\phi}}{\longrightarrow} G_{t_{i}, x} \stackrel{q}{\longrightarrow} G_{i, j, x}$ (q= quotient map) is equal to $\phi$ of 3.2.5. Define a map $\hat{f}: \mathbf{R} \rightarrow E$ by $\hat{f}(t)=\hat{\phi}(t)(\hat{z})$ for all $t \in \mathbf{R}$. Then $\hat{f}$ satisfies the following properties. (Note that 3.2.6(b) is a consequence of 3.2.6(a), and Appendix 2, and the fact that $f$ has unit speed.)

3.2.6. (a) $\rho \circ \hat{f}=f$.

(b) $\hat{f}$ has constant speed $\geq 1$.

Note that Properties 3.2.5(b) and 3.2.6(a) together imply that:

3.2.7. (a) $d_{E}^{\prime}(g(\hat{z}), \hat{f}(u)) \geq \epsilon / 8$, for some $u \in\left(0, t_{i, j, 2}\right)$ and all $g \in C_{i, j, x}$.

Combining 3.2.6(b) with 3.2.7(a) (with $g=$ id in 3.2.7(a)) we get that:

3.2.7. (b) $\hat{f}$ has constant speed $\geq \epsilon / 8 t_{i, j, 2}$.

Now, combining 3.2.6 and 3.2.7(b), together with the relation:

3.2.7. (c) $t_{i, j, 2} \ll \epsilon\left(t_{i, j, 1}\right)^{\operatorname{dim} E+2}$

(which follows from 3.0) and the fact that $\rho \circ \hat{f}$ has unit speed, imply that the following is true:

3.2.8. (a) There is $s \in\left(u, t_{i, j, 1} / 2\right)$ so that $d_{E}^{\prime}(\hat{f}(0), \hat{f}(s)) \ll t_{i, j, 1}$.

[In more detail we verify 3.2.8(a) as follows: Let $B\left(z, t_{i, j, 1}\right)$ denote the open ball in $\hat{V}$ of radius $t_{i, j, 1}$ centered at $z$. We may choose a covering $\left\{B_{k}: 1 \leq\right.$ $k \leq \kappa\}$ for $\rho^{-1}\left(B\left(z, t_{i, j, 1}\right)\right)$ by open balls $B_{k}$ in $E$ satisfying:

(i) $\operatorname{radius}\left(B_{k}\right) \ll t_{i, j, 1}$,

(ii) $\kappa \ll t_{i, j, 1}^{-\operatorname{dim} E}$. 
On the other hand it follows from 3.2.6, and from the fact that $f$ has unit speed, that:

(iii) $\hat{f}(a) \in \bigcup_{k=1}^{\kappa} B_{k}$ for all $a \in\left[0, t_{i, j, 1}\right]$.

Finally, by combining (ii) and (iii) above with Property 3.2.7(c), we deduce that there are numbers $a, c \in\left[0, t_{i, j, 1} / 2\right]$ such that:

(iv) $a+t_{i, j, 2}<c$ and $\hat{f}(a), \hat{f}(c) \in B_{k}$ for some $1 \leq k \leq \kappa$.

Now Property 3.2.8(a) is an immediate consequence of (i) and (iv), and of the inequality $u<t_{i, j, 2}$ (cf. 3.2.7(a)), provided the number $s$ of 3.2.8(a) is defined by $s=c-a$.]

Note that Appendix 2, 2.2, and 3.1.1 assure that all the hypotheses for $2.7(\mathrm{~d})$ are satisfied by the action $G_{t_{i}, x} \times E \rightarrow E$. Thus we may apply $2.7(\mathrm{~d})$, in conjunction with 3.2.8(a), to deduce that:

3.2.8. (b) $d_{G_{t_{i}, x}}(\hat{\phi}(0), \hat{\phi}(s))<t_{i, j, 1}$.

Now we can apply Lemma 2.8 with $\phi_{1}, t, s, G$ of 2.8 set equal to $\hat{\phi}, t_{i, j, 1}, s$ (of 3.2.8), $G_{t_{i}, x}$, and with $g_{G}$ of 2.8 set equal to the Riemannian metric on $G_{t_{i}, x}$ induced from $g_{E}^{\prime}$ by identifying $G_{t_{i}, x}$ with the subset $G_{t_{i}, x}(\hat{z})=\{g(\hat{z})$ : $\left.g \in G_{t_{i}, x}\right\}$ of $E$ via the map $g \rightarrow g(\hat{z})$. We choose the local angle control numbers $(\alpha, \beta)$ (assumed in the hypothesis of 2.8) to be equal $\left(\lambda \delta_{1}, \delta_{1}\right)$ where $\lambda>1$ comes from Remark 2.2 and A.2.5; then it follows from 2.2 and A.2.5 that the left action $G \times G \rightarrow G$ has local angle control equal $(\alpha, \beta)$ as required in 2.8. Note that 3.2.8(b), and the preceding remarks, imply that these choices for $\phi_{1}, t, s, G, g_{G}$ satisfy Property 2.8(a). Thus we may apply Lemma 2.8 to conclude that there is another one-parameter group $\phi_{2}: \mathbf{R} \rightarrow G_{t_{i}, x}$ which satisfies the following properties:

3.2.9. (a) $\phi_{2}(s)(\hat{z})=\hat{z}$.

(b) $d_{E}^{\prime}\left(\phi_{2}(u)(\hat{z}), \phi_{1}(u)(\hat{z})\right)<(u / s) 4^{\operatorname{dim} E}\left(t_{i, j, 1}\right)$ for all $u \in[0, s]$.

We can deduce from 3.2.9(b), 3.2.7(a), and from the inequality $t_{i, j, 1} \ll \epsilon / 8$ (cf. 3.0), the following additional property for $\phi_{2}$ :

3.2.9. (c) Image $\left(\phi_{2}\right)$ is not contained in $C_{i, j, x}$.

Note that 3.2.9(a), together with the fact that $G_{t_{i}, x} \times E \rightarrow E$ is a free action [the action $H \times \hat{V} \rightarrow \hat{V}$ is effective (cf. Appendix 2) and thus the action $H \times E \rightarrow E$ is free] implies that Image $\left(\phi_{2}\right)$ is a compact subgroup of $G_{t_{i}, x}$. Let $C$ denote the minimal compact subgroup of $G_{t_{i}, x}$ which contains $C_{i, j+1, x} \cup \operatorname{Image}\left(\phi_{2}\right)$. The following properties hold:

3.2.10. (a) $C_{i, j, x} \subset C \subset G_{t_{i}, x}$ and $C_{i, j, x} \neq C$. 
(b) $d_{\hat{V}}^{\prime}(\rho(x), h \rho(x))<t_{i, j}$, for all $h \in C$.

Note that 3.2.10(a) is a consequence of 3.1.3, 3.2.9(c) and of the definition of $C$. Also note that each element $h \in C$ can be written as product $h=g_{1} g_{2}$ of two elements in $C_{i, j+1, x} \cup \operatorname{Image}\left(\phi_{2}\right)$ (because $G_{t_{i}, x}$ is connected and nilpotent). Thus Property 3.2.10(b) follows from 3.0, 3.1.2 (as applied to $\left.C_{i, j+1, x}\right)$, and from the following property:

3.2.11. $d_{\hat{V}}^{\prime}(\rho(x), g \rho(x))<8^{\operatorname{dim} E}\left(t_{i, j, 1}\right)$ for all $g \in \operatorname{Image}\left(\phi_{2}\right)$.

To verify 3.2.11 we note first that $\rho: E \rightarrow \hat{V}$ doesn't increase distances; thus

$$
d_{\hat{V}}^{\prime}\left(\phi_{2}(u) z, \phi_{1}(u) z\right)<(u / s) 4^{\operatorname{dim} E}\left(t_{i, j, 1}\right), \text { for all } u \in(0, s),
$$

follows from 3.2.9(b). Now $\phi_{1}(u) z$ has unit speed in the variable $u$ (because it coincides with the path $f(u))$, and $s \in\left(0, t_{i, j, 1}\right)$ (by 3.2.8(a)), so

$$
d_{\hat{V}}^{\prime}\left(z, \phi_{1}(u) z\right)<t_{i, j, 1} \text { for all } u \in(0, s) .
$$

On the other hand we deduce from 3.0, 3.2.1(a), and from the fact that $z \in B\left(y, t_{i, j, 2}\right)$, that

$$
d_{\hat{V}}^{\prime}(\rho(x), z)<t_{i, j, 1} .
$$

Now Property 3.2.11 follows directly form 3.2.9(a), and from the three preceding inequalities.

Note that 3.2.10 contradicts the maximality of the compact subgroup $C_{i, j, x}$. This contradiction can be traced back to our assumption that the action $G_{i, j, x} \times F_{i, j, k, y} \rightarrow F_{i, j, k, y}$ does not have local control equal $\left(\epsilon, t_{i, j, 2}\right)$ at $z \in B\left(y, t_{i, j, 2}\right)$ (cf. 3.2.5).

This completes the verification of Claim 3.2.3.

\section{Verification of Claim 3.2.4.}

We begin by verifying the following property:

3.2.12. diameter $\left(L / \Lambda_{i, j, k, x}^{\prime}\right)<2 \tau t_{i+1}$, for each leaf $L \in \mathcal{G}_{i, j, k, y}$.

Here $\tau>0$ comes from 2.7(c), and the diameter is computed with respect to the Riemannian metric which $L \subset \hat{V}$ inherits from $\left(\hat{V}, g_{\hat{V}}^{\prime}\right)$.

It will suffice to consider only those leaves $L \in \mathcal{G}_{i, j, k, x}$ in 3.2 .12 which intersect with the subset $B\left(y ; t_{i, j, k}\right)$ of $F_{i, j, x}$ (cf. 3.1.5, and review the definition of $\left.F_{i, j, k, y}\right)$; note that this condition on $L$ is equivalent to:

3.2.13. (a) $d_{\hat{V}}^{\prime}(y, L)<t_{i, j, k}$,

since $F_{i, j, x}$ is a path component of the fixed point set of $C_{i, j, x} \times \hat{V} \rightarrow \hat{V}$ and the injectivity radius of $\hat{V}$ at $y$ is $\gg t_{i, j, k}$ (cf. 3.0 and Appendix 2). Let $\mathcal{G}_{i, x}$ 
denote the foliation of $E$ by the orbits of the group action $G_{t_{i}, x} \times E \rightarrow E$ (recall that this action is free). Note that the pre-image of $L$ under the composition map $E \stackrel{\rho}{\longrightarrow} \hat{V}$ is a union of leaves in $\mathcal{G}_{i, x}$. It follows from Appendix 2, 3.0, 3.2.1(a), and from 3.2.13(a) that there is a leaf $\hat{L} \in \mathcal{G}_{i, x}$ such that $\rho(\hat{L})=L$ and:

3.2.13. (b) $d_{E}^{\prime}(x, \hat{L})<2 t_{i, j, k}$.

Remark 2.2 and A.2.5 assure us that we may apply Lemma 2.6, with the group action $G \times N \rightarrow N$ and the numbers $\alpha, \beta$ of 2.6 taken to be the group action $G_{t_{i}, x} \times E \rightarrow E$ and the numbers $\lambda t, t$ (for any small $t>0$ and $\lambda$ as in 2.2 and A.2.5), to conclude that:

3.2.13. (c) $K\left(\mathcal{G}_{i, x} ; E\right)<10^{6} \lambda$.

Now 3.2.13(b) and (c) and 3.1.14 together imply that:

3.2.13. (d) diameter $\left(\hat{L} / \Lambda_{i, j, k, x}^{\prime}\right)<2 \tau t_{i+1}$.

[When deriving 3.2.13(d) see also 3.0, 3.1.1, and recall that the actions of $G_{t_{i}}$ and $\Lambda_{i, j, k, x}$ on $E$ are free.] Since $\rho: E \rightarrow \hat{V}$ is a Riemannian submersion which maps $\hat{L}$ onto $L$, and which commutes the action of $\Lambda_{i, j, k, x}^{\prime}$ on $E$ and on $\hat{V}$, we may deduce 3.2.12 from 3.2.13(d).

It follows from A.2.2 and from 3.0 that the radius of injectivity for $F_{i, j, x}$ is greater than $t_{i, j, 2}$ at $y \in F_{i, j, x}$. This fact, together with the curvature restrictions placed on $\mathcal{G}_{i, j, 2, y}$ by 3.2 .3 , allow us to define a "local projection"

$$
\pi: X\left(y ; t_{i, j, 2}\right) \rightarrow A^{\perp}\left(y ; t_{i, j, 2}\right)
$$

from an open subset $X\left(y ; t_{i, j, 2}\right) \subset F_{i, j, 2, y}$ as follows: Recall that $B^{\perp}(y ; t)$ and $A^{\perp}(y ; t)$ were defined just prior to 3.2 .4 above for any $t \in\left(0, t_{i, j, 2}\right] ; A^{\perp}(y ; t)$ is a subset of $T\left(F_{i, j, 2}\right)_{y}$ and $B^{\perp}(y ; t) \subset F_{i, j, 2}$ is the diffeomorphic image of $A^{\perp}(y ; t)$ under the exponential map exp : $A^{\perp}(y ; t) \rightarrow F_{i, j, 2}$. For each $q \in B^{\perp}(y ; t)$ let $L_{q}$ denote the leaf of $\mathcal{G}_{i, j, 2, y}$ containing $q$ and let $X(y ; t)_{q}$ denote the open ball in $L_{q}$ of radius $t$ centered at $q$. Set

$$
X(y ; t)=\bigcup_{q \in B^{\perp}(y ; t)} X(y ; t)_{q}
$$

for each $t \in\left(0, t_{i, j, 2}\right]$; and define $\pi: X\left(y ; t_{i, j, 2}\right) \rightarrow A^{\perp}\left(y ; t_{i, j, 2}\right)$ by sending $X\left(y ; t_{i, j, 2}\right)_{q}$ to $\exp ^{-1}(q)$ for each $q \in B^{\perp}\left(y ; t_{i, j, 2}\right)$. Then $\pi$ is a smooth fiber bundle projection having the open balls $\left\{X\left(y ; t_{i, j, 2}\right)_{q}: q \in B^{\perp}(y ; t)\right\}$ for fibers. Note that for each $t \in\left(0, t_{i, j, 2}\right]$ the restricted map $\pi: X(y, t) \rightarrow$ $A^{\perp}(y ; t)$ is a smooth bundle projection having the open balls $\left\{X(y ; t)_{q}: q \in\right.$ $\left.B^{\perp}(y ; t)\right\}$ for fibers. 
If Claim 3.2.4 does not hold then we may deduce from 3.2.12 that there is $g \in \Lambda_{i, j, k, x}^{\prime}$ which for some $z \in X\left(y ; t_{i, j, k-1}\right)$ satisfies:

3.2.14. (a) $d_{F_{i, j, x}}^{\prime}(z, g(z))<4 t_{i, j, k-1}$, and thus $g(z) \in X\left(y ; t_{i, j, 2}\right)$;

(b) $\pi(z) \neq \pi(g(z))$.

We will complete the proof for 3.2.4 by deriving a contradiction to 3.2.14(b).

We can deduce from 3.0, 3.2.14(a), and from the definition of the $\pi$, that for each positive integer $s \leq t_{i, j, k-2}^{-\operatorname{dim} E}$ left multiplication by $g^{s}$ is a smooth embedding

$$
g^{s}: X\left(y ; t_{i, j, 2} / 8\right) \rightarrow X\left(y ; t_{i, j, 2}\right)
$$

which maps each fiber for $\pi \mid X\left(y ; t_{i, j, 2} / 8\right)$ into a fiber for $\pi$. Thus each $g^{s}$ induces a smooth embedding $h_{s}: A^{\perp}\left(y ; t_{i, j, 2} / 8\right) \rightarrow A^{\perp}\left(y ; t_{i, j, 2}\right)$. We will verify the following properties for $\left\{h_{s}: 1 \leq s \leq t_{i, j, k-2}^{-\operatorname{dim} E}\right\}$ :

3.2.15. (a) $\left|h_{s}(0)\right| \ll t_{i, j, k-2}$.

(b) There is linear isometry $I_{s}: T^{\perp}\left(\mathcal{G}_{i, j, 2, y}\right)_{y} \rightarrow T^{\perp}\left(\mathcal{G}_{i, j, 2, y}\right)_{y}$ such that $\left|D h_{s}-I_{s}\right|<\mathcal{O}(\epsilon)$. (Here $T^{\perp}\left(\mathcal{G}_{i, j, 2, y}\right)$ denotes the orthogonal complement for $T \mathcal{G}_{i, j, 2, y}$ in $T F_{i, j, 2, y}$.)

(c) If $h_{s} \mid A^{\perp}\left(y ; t_{i, j, 2} / 8^{2}\right)$ has a fixed point then $h_{s}$ is the inclusion map.

(d) Each composition $h_{1}^{s}$ is well-defined and equal to $h_{s}$ on all of $A^{\perp}$ (y; $\left.t_{i, j, 2} / 8^{2}\right)$.

Properties 3.2.15(a) and (b) are a consequence of 3.0 and 3.2.14(a), and of the curvature condition and the "strongly Riemannian" condition placed on the foliation of $X\left(y ; t_{i, j, 2}\right)$ by the fibers of $\pi$ by Claim 3.2.3 (see also Appendix 1). And Property 3.2.15(d) is a consequence of 3.2.15(a) and (b). Towards verifying Property 3.2.15(c) we denote by $q \in A^{\perp}\left(y ; t_{i, j, 2} / 8^{2}\right)$ a fixed point for $h_{s}$; we have then that

$$
g^{s}\left(\pi^{-1}(q) \cap X\left(y ; t_{i, j, 2} / 8^{2}\right)\right) \subset \pi^{-1}(q) ;
$$

and then by appealing to 3.0 and 3.2.14(a) this last assertion can be improved to

$$
g^{s}\left(\pi^{-1}(q) \cap X\left(y ; t_{i, j, 2} / 8^{2}\right)\right) \subset \pi^{-1}(q) \cap X\left(y ; t_{i, j, 2} / 8\right) .
$$

It follows from this last inclusion, and from the fact that $G_{i, j, x} \times F_{i, j, 2, y} \rightarrow$ $F_{i, j, 2, y}$ is a free action by isometries (cf. 3.2.3(b)), that there is a path $f$ : $[0,1] \rightarrow G_{i, j, x}$ which satisfies:

3.2.16. (a) $f(0)=$ identity and $f(1)=g^{s}$;

(b) $f(t)\left(\pi^{-1}(q) \cap X\left(y ; t_{i, j, 2} / 8^{2}\right)\right) \subset \pi^{-1}(q) \cap X\left(y ; t_{i, j, 2} / 4\right)$ for all $t \in[0,1]$. 
Now it follows from 3.2.16(b), and from the curvature condition placed on the foliation of $X\left(y ; t_{i, j, k-2}\right)$ by the fibers of $\pi$ in Claim 3.2.3, that:

3.2.16. (c) $f(t)\left(\pi^{-1}(u) \cap X\left(y ; t_{i, j, 2} / 8^{2}\right)\right) \subset \pi^{-1}(u)$ for all $u \in A^{\perp}\left(y ; t_{i, j, 2} / 8^{2}\right)$ and all $t \in[0,1]$.

Property 3.2.15(c) is a consequence of 3.2.16(a) and (c).

A contradiction to Property 3.2.14(b) is contained in the following statement:

3.2.17. All the maps $\left\{h_{s}: 1 \leq s \leq t_{i, j, k-2}^{-\operatorname{dim} E}\right\}$ have a common fixed point in $A^{\perp}\left(y ; t_{i, j, 2} / 8^{2}\right)$.

In fact 3.2.17 and 3.2.15(c) together imply that $h_{1}$ is the inclusion map; this is equivalent to the equalities $\pi(z)=\pi(g(z))$ for all $z \in X\left(y ; t_{i, j, 2} / 8^{2}\right)$, which contradicts 3.2.14(b).

Thus to complete the proof of Claim 3.2.4 it remains only to verify 3.2.17. First we will verify the following weakened version of 3.2.17:

3.2.18. For some positive integer $r<t_{i, j, k-2}^{-\operatorname{dim} \mathrm{E}}$ we have that $h_{r}(0)=0$.

Towards this end we first note it follows from 3.2.14(a), 3.0, 3.2.1(a) that:

3.2.19. (a) $d_{\hat{V}}^{\prime}\left(\rho(x), g^{s}(\rho(x))\right)<10 s t_{i, j, k-1}$

for all $s \in\left\{1,2, \ldots, t_{i, j, k-2}^{-\operatorname{dim} \text { E }}\right\}$, where $x \in E$ was selected at the outset of 3.1. Now it follows from 3.2.19(a) and 3.0 that there are two distinct positive integers $r_{1}<r_{2} \leq t_{i, j, k-2}^{-\operatorname{dim} E}$ such that:

3.2.19. (b) $d_{E}^{\prime}\left(g^{r_{1}}(x), g^{r_{2}}(x)\right) \ll t_{i, j, k-2}$.

[We use a covering argument to verify 3.2.19, similar to the argument of 3.1.11 used above in the proof of Claim 3.1.5(c).] Now, if we set $r=r_{2}-r_{1}$, we have (by 3.2.19(b)) that:

3.2.19. (c) $d_{E}^{\prime}\left(x, g^{r}(x)\right) \ll t_{i, j, k-2}$.

Combining this last property with Lemma 2.7(d) (where we set the $N, G, \Gamma, t$ of 2.7 equal to $E, G_{t_{i}, x}, \Lambda_{i, j, k, x}^{\prime}$, maximum $\left.\left\{d_{E}^{\prime}\left(x, g^{r}(x)\right), t_{i, j, k}\right\}\right)$ we can deduce the following:

3.2.19.

(d) There is a one-parameter subgroup $f: \mathbf{R} \rightarrow G_{t_{i}, x}$ such that $g^{r} \in$ $f([0,1])$ and such that $d_{\left(G_{t_{i}, x}\right) x}^{\prime}(x, f(t)(x)) \ll t_{i, j, k-2}$ for all $t \in[0,1]$.

We can combine 3.2.19(d) with 3.2.1(a), and with the fact that $\rho: E \rightarrow \hat{V}$ is a Riemannian submersion, to conclude: 
3.2.19. (e) $d_{L_{y}}^{\prime}(y, f(t)(y)) \ll t_{i, j, k-2}$ for all $t \in[0,1]$.

Now this last inequality, together with the definition of $X(y ; t)_{q}$ as the open ball in $L_{y}$ of radius $t$ and center $y$, implies that all the points $\{f(t)(y): t \in$ $[0,1]\}$ are contained in the fiber $\pi^{-1}(0)$. Since $g^{r} \in f([0,1])$ it follows that $g^{r}(y) \in \pi^{-1}(0)$; from which we immediately deduce 3.2.18.

Now to complete the verification for 3.2.17 we note that it follows from 3.0, 3.2.18, and 3.2.15(a)-(d), that there is a cyclic group action $\psi: \mathbf{Z}_{r} \times W \rightarrow W$, where

$$
A^{\perp}\left(y ; t_{i, j, 2} / 8^{3}\right) \subset W \subset A^{\perp}\left(y ; t_{i, j, 2} / 8^{2}\right)
$$

and

$$
\psi(s, w)=h_{s}(w) \text { for } 1 \leq s \leq r .
$$

By appealing to 3.2.15(a) and (b) we can obtain a fixed point for the action $\psi: \mathbf{Z}_{r} \times W \rightarrow W$ as the limit of a sequence of points $z_{1}, z_{2}, z_{3}, \ldots$ defined inductively by

$$
\begin{aligned}
z_{1} & =0, \\
z_{n+1} & =r^{-1} \sum_{s=1}^{r} \psi\left(s, z_{n}\right) .
\end{aligned}
$$

We note that $\operatorname{limit}_{n \rightarrow \infty} z_{n}$ is the desired common fixed point of 3.2.17.

This completes the verification for Claim 3.2.4.

3.3. The projection $\pi_{i, j, k, y}: \bar{F}_{i, j, k, y} \rightarrow B_{i, j, k, y}$ for any $4 \leq k<(m+$ $4)^{2}-1$. Set

$$
\begin{aligned}
\bar{\Lambda}_{i, j, k, x} & =\Lambda_{i, j, k, x} / \Lambda_{i, j, k, x}^{\prime}, \\
\bar{V} & =\hat{V} / \Lambda_{i, j, k, x}^{\prime}, \\
\bar{F}_{i, j, k, y} & =F_{i, j, k, y} / \Lambda_{i, j, k, x}^{\prime}, \\
\overline{\mathcal{G}}_{i, j, k, y} & =\mathcal{G}_{i, j, k, y} / \Lambda_{i, j, k, x}^{\prime}, \\
\bar{y} & =q_{i, j, k, y}(y),
\end{aligned}
$$

where $q_{i, j, k, y}: F_{i, j, k, y} \rightarrow \bar{F}_{i, j, k, y}$ is the quotient map and $y$ comes from 3.2.1(a). We will verify that the following properties hold:

3.3.1. For each $4 \leq k<(m+4)^{2}-1$ we have:

(a) $\overline{\mathcal{G}}_{i, j, k, y}$ is a strongly Riemannian foliation which satisfies

$$
K\left(\overline{\mathcal{G}}_{i, j, k, y} ; \bar{F}_{i, j, k, y}\right)<10^{6} \epsilon t_{i, j, 2}^{-1} .
$$

(b) diameter $(L)<2 \tau t_{i+1}$, for each leaf $L \in \overline{\mathcal{G}}_{i, j, k, y}$.

(c) diameter $\left(\bar{F}_{i, j, k, y}\right) \ll t_{i, j, k-1, l}$, where $l=(m+4)^{2}-1$.

(d) $\bar{F}_{i, j, k+1, y} \subset \bar{F}_{i, j, k, y}$. 
(e) $\overline{\mathcal{G}}_{i, j, k, y} \mid \bar{F}_{i, j, k+1, y}=\overline{\mathcal{G}}_{i, j, k+1, y}$.

Property 3.3.1(a) is a direct consequence of 3.2.3(c). Property 3.3.1(b) is a direct consequence of 3.2.12. Properties 3.3.1(d) and (e) follow from 3.1.1 and 3.1.5(d), and from the fact that $F_{i, j, k+1, y} \subset F_{i, j, k, y}$. Now we will verify 3.3.1(c). Since $\Lambda_{i, j, k, x}^{\prime}$ is a normal subgroup of $\Lambda_{i, j, k, x}$. (cf. 3.1.5(c)) it follows that $\bar{\Lambda}_{i, j, k, x}$ defined above is a group. We may choose, by 3.0, 3.1.4, 3.1.5(c), 3.2.1(a), a subset of elements $\left\{g_{1}, g_{2}, \ldots, g_{\lambda}\right\} \subset \Lambda_{i, j, k, x}$ which satisfy the following properties:

3.3.2. (a) $\left\{g_{1}, \ldots, g_{\lambda}\right\}$ maps onto $\bar{\Lambda}_{i, j, k, x}$ under the quotient map $\Lambda_{i, j, k, x} \rightarrow$ $\bar{\Lambda}_{i, j, k, x}$.

(b) $d_{\hat{V}}\left(y, g_{i}(y)\right)<\left(t_{i}^{-2 \operatorname{dim} E}\right)\left(2 t_{i, j, k}\right)$.

Let $U_{i, j, k, y}$ denote the union of all leaves in $\mathcal{G}_{i, j, k, y}$ which intersect the ball $B\left(y ; t_{i, j, k}\right)$ (cf. 3.2); set

$$
\bar{U}_{i, j, k, y}=U_{i, j, k, y} / \Lambda_{i, j, k, x}^{\prime} .
$$

We note it follows from 3.3.1(b), and from the fact that $B\left(y ; t_{i, j, k}\right)$ has radius equal $t_{i, j, k}$, that the following holds:

3.3.2. (c) diameter $\left(\bar{U}_{i, j, k, y}\right)<(4 \tau+2) t_{i, j, k}$.

Since we have that $F_{i, j, k, y}=\bigcup_{g \in \Lambda_{i, j, k, x}} g\left(U_{i, j, k, y}\right)$ (cf. 3.2), we also have that:

3.3.2. (d) $\bar{F}_{i, j, k, y}=\bigcup_{t=1}^{\lambda} g_{t}\left(\bar{U}_{i, j, k, y}\right)$.

Now we deduce 3.3.1(c) from 3.3.2(b)-(d) and from 3.0. This completes the verification for 3.3.1.

Now we can use 3.3.1 to aid in the definition of the projections $\pi_{i, j, k, x}$ : $\bar{F}_{i, j, k, y} \rightarrow B_{i, j, k, y}$ for any $4 \leq k<(m+4)^{2}-1$. Let $W$ denote the space of all $v \in T\left(\bar{F}_{i, j, k, y}\right)_{\bar{y}}$ which are perpendicular to $\overline{\mathcal{G}}_{i, j, k, y}$, and let $\exp : W \rightarrow \bar{F}_{i, j, x}$ and $f_{W}: W \rightarrow \mathbf{R}^{\beta}$ denote the exponential map and a fixed linear isometry respectively, where $\bar{F}_{i, j, x}$ is defined by

$$
\bar{F}_{i, j, x}=F_{i, j, x} / \Lambda_{i, j, k, x}^{\prime}
$$

and where $\beta=\operatorname{dim} W$ (cf. 3.2.1(c) and 3.1.5(d)). Let $B\left(2 t_{i}\right) \subset W$ denote the ball of radius $2 t_{i}$ centered at the origin of $W$. Since the injectivity radius of $F_{i, j, x}$ is much greater than $2 t_{i}$ at every one of its points (cf. A.2.2, 3.0) it follows from 3.3.1(a)-(c) and from 3.2.4 that $\exp : B\left(2 t_{i}\right) \rightarrow \bar{F}_{i, j, x}$ maps $B\left(2 t_{i, j, 3,1}\right)$ diffeomorphically onto a smooth submanifold $\bar{B}\left(2 t_{i, j, 3,1}\right)$ of 
$\bar{F}_{i, j, x}$ which intersects each leaf $L \in \overline{\mathcal{G}}_{i, j, k, y}$ in exactly one point (see 3.0, and recall that $\Lambda_{i, j, k, x}^{\prime}$ leaves each leaf of $\mathcal{G}_{i, j, k, x}$ invariant). Define a map $\pi_{i, j, k, y}: \bar{F}_{i, j, k, y} \rightarrow B_{i, j, k, y}$ by:

3.3.3. (a) $\pi_{i, j, k, y}(L)=f_{W} \circ \exp ^{-1}\left(L \cap \bar{B}\left(2 t_{i, j, 3,1}\right)\right)$ for $L \in \overline{\mathcal{G}}_{i, j, k, y}$.

(b) $B_{i, j, k, y}=\operatorname{Image}\left(\pi_{i, j, k, y}\right)$.

There is also a canonical cross section $s_{i, j, k, y}: B_{i, j, k, y} \rightarrow \bar{F}_{i, j, k, y}$ defined by:

3.3.3. (c) $s_{i, j, k, y}(v)=\exp \circ f_{W}^{-1}(v)$ for $v \in B_{i, j, k, y}$.

Note it follows from 3.3.1(d) and (e) that $W$ (in the preceding construction) is independent of $k$. Thus we concluded (by 3.3.1(d) and (e)) that the following relations hold:

3.3.4. (a) $\pi_{i, j, k, y} \mid \bar{F}_{i, j, k+1, y}=\pi_{i, j, k+1, y}$.

(b) $B_{i, j, k+1, y} \subset B_{i, j, k, y}$.

(c) $s_{i, j, k, y} \mid B_{i, j, k+1, y}=s_{i, j, k+1, y}$.

Also, since $F_{i, j, k, y}$ contains the open ball $B\left(y ; t_{i, j, k}\right)$, it follows that $\bar{F}_{i, j, k, y}$ contains the subset $\bar{B}\left(t_{i, j, k}\right)$, where $\bar{B}\left(t_{i, j, k}\right)=\exp \left(B\left(t_{i, j, k}\right)\right)$ and where $B\left(t_{i, j, k}\right)$ is the open ball of radius $t_{i, j, k}$ centered at the origin of $W$. The following property is a reinterpretation of the containment $\bar{B}\left(t_{i, j, k}\right) \subset \bar{F}_{i, j, k, y}$ and of Property 3.3.1(c):

\subsection{5.}

$B_{i, j, k, y}$ is an open subset of $\mathbf{R}^{\beta}$ which contains the open ball of radius $t_{i, j, k}$ centered at the origin of $\mathbf{R}^{\beta}$; moreover diameter $\left(B_{i, j, k, y}\right) \ll$ $t_{i, j, k-1, l}$ with $l=(m+4)^{2}-1$.

We note that if $\bar{y} \in \bar{F}_{i, j, k, y}$ were replaced by any other point $\bar{y}^{\prime} \in \bar{F}_{i, j, k, y}$ in the preceding construction for the maps $\pi_{i, j, k, y}$ and $s_{i, j, k, y}$ we would obtain a new projection $\pi_{i, j, k, y}^{\prime}: \bar{F}_{i, j, k, y} \rightarrow B_{i, j, k, y}^{\prime}$ and a new cross section $s_{i, j, k, y}^{\prime}$ : $B_{i, j, k, y}^{\prime} \rightarrow \bar{F}_{i, j, k, y}$. We will verify the following properties:

3.3.6. Set $s=\pi_{i, j, k, y} \circ s_{i, j, k, y}^{\prime}$; note that $s$ maps the open subset $B_{i, j, k, y}^{\prime} \subset \mathbf{R}^{\beta}$ diffeomorphically onto to the open subset $B_{i, j, k, y} \subset \mathbf{R}^{\beta}$. For each $z \in B_{i, j, k, y}^{\prime}$ there is a linear isometry $L_{z}: \mathbf{R}^{\beta} \rightarrow \mathbf{R}^{\beta}$ such that $s$ and $L_{z}$ satisfy:

(a) $\left\|D s_{\mid z}-L_{z}\right\| \ll \epsilon$;

(b) $\left\|D^{2} s_{\mid z}\right\|<\mathcal{O}(\epsilon) t_{i, j, 2}^{-1}$.

Towards this end we let $W^{\prime}$ denote all $v \in T\left(\bar{F}_{i, j, k, x}\right)_{\bar{y}^{\prime}}$ which are perpendicular to $\overline{\mathcal{G}}_{i, j, k, y}$, and let $f_{W^{\prime}}: W^{\prime} \rightarrow \mathbf{R}^{\beta}$ denote a given linear isometry; we use $W^{\prime}, f_{W^{\prime}}$ in the preceding construction (in place of $W, f_{W}$ ) to obtain 
$\pi_{i, j, k, y}^{\prime}$ and $s_{i, j, k, y}^{\prime}$ (in place of $\pi_{i, j, k, y}$ and $s_{i, j, k, y}$ ). For any given $z \in B_{i, j, k, y}^{\prime}$ we set $z_{1}=s_{i, j, k, y}^{\prime}(z)$ and set $z_{2}=\pi^{\prime-} 1_{i, j, k, y}(z) \cap \operatorname{Image}\left(s_{i, j, k, y}\right)$; let $W_{i}$ denote all vectors $v \in T\left(\bar{F}_{i, j, k, y}\right)_{z_{i}}$ which are perpendicular to $\overline{\mathcal{G}}_{i, j, k, y}$; and choose linear isometries $f_{W_{i}}: W_{i} \rightarrow \mathbf{R}^{\beta}$. For sufficiently small neighborhoods $U^{\prime}, U, U_{1}, U_{2}$ of $z, s(z), 0,0$ in $\mathbf{R}^{\beta}$ there are diffeomorphisms

$$
g_{3}: U^{\prime} \rightarrow U_{1} \text { and } g_{2}: U_{1} \rightarrow U_{2} \text { and } g_{1}: U_{2} \rightarrow U
$$

defined as follows: Define $g_{1}$ to be the composition of three maps $g_{1}=$ $g_{1,1} \circ g_{1,2} \circ g_{1,3}$. Here $g_{1,1}^{-1}=\exp \circ\left(f_{W}^{-1} \mid U\right) ; g_{1,3}=\exp \circ\left(f_{W_{2}}^{-1} \mid U_{2}\right)$; and $g_{1,2}:$ Image $\left(g_{1,3}\right) \rightarrow \operatorname{Image}\left(g_{1,1}^{-1}\right)$ is the holonomy map for $\overline{\mathcal{G}}_{i, j, k, y}$. Define $g_{2}$ to be the composition of three maps $g_{2}=g_{2,1} \circ g_{2,2} \circ g_{2,3}$ where $g_{2,1}=$ $g_{1,3}^{-1} ; g_{2,3}=\exp \circ\left(f_{W_{1}}^{-1} \mid U_{1}\right) ;$ and $g_{2,2}: \operatorname{Image}\left(g_{2,3}\right) \rightarrow \operatorname{Image}\left(g_{1,3}\right)$ is the holonomy map for $\overline{\mathcal{G}}_{i, j, k, y}$. Define $g_{3}$ to be the composition of three maps $g_{3}=g_{3,1} \circ g_{3,2} \circ g_{3,3}$ where $g_{3,1}=g_{2,3}^{-1} ; g_{3,3}=\exp \circ\left(f_{W^{\prime}}^{-1} \mid U^{\prime}\right)$; and $g_{3,2}$ : Image $\left(g_{3,3}\right) \rightarrow \operatorname{Image}\left(g_{2,3}\right)$ is just the holonomy map for $\overline{\mathcal{G}}_{i, j, k, y}$. Note that:

3.3.7. (a) $s \mid U^{\prime}=g_{1} \circ g_{2} \circ g_{3}$.

It follows from the fact that $\overline{\mathcal{G}}_{i, j, k, y}$ is "strongly Riemannian" (cf. 3.3.1(a)) that:

3.3.7. (b) $g_{2}: U_{1} \rightarrow U_{2}$ is an isometry.

On the other hand we will show that there are linear isometries $I_{1}, I_{3}: \mathbf{R}^{\beta} \rightarrow$ $\mathbf{R}^{\beta}$ which satisfy the following claim in which $\hat{1}=0$ and $\hat{3}=z$ :

Claim 3.3.8. (a) $\left\|D^{1}\left(g_{b}-I_{b}\right)_{\mid \hat{b}}\right\| \ll \epsilon$ for $b=1,3$.

(b) $\left\|D^{2}\left(g_{b}-I_{b}\right)_{\mid \hat{b}}\right\|<\mathcal{O}(\epsilon) t_{i, j, 2}^{-1}$ for $b=1,3$.

Now Properties 3.3.6(a) and (b) are a consequence of Properties 3.3.7(a) and (b) and 3.3.8(a) and (b).

\section{Verification of Claim 3.3.8.}

We will verify the claim for $b=1$; the same proof works for $b=3$.

First we remark that $F_{i, j, k, y}$ is an open subset of $F_{i, j, x}$, and that $F_{i, j, x}$ is (locally) a totally geodesic subset of $\hat{V}$ with respect to the Riemannian metric $g_{\hat{V}}^{\prime}$. Thus, if $h$ denotes the Riemannian metric inherited by $F_{i, j, k, y}$ from $g_{\hat{V}}^{\prime}$, we see (cf. Appendix 2) that $F_{i, j, k, y}, h$ is an $A^{\prime}$-regular Riemannian manifold, where $A^{\prime}$ comes from A.2.2. Note that the quotient $\bar{h}$ of the metric $h$ by the action $\Lambda_{i, j, k, x}^{\prime} \times F_{i, j, k, y} \rightarrow F_{i, j, k, y}$ is also an $A^{\prime}$-regular metric for $\bar{F}_{i, j, k, y}$. Thus, for sufficiently small $\bar{\epsilon}>0$, there is an imbedding $f_{p}: B_{\bar{\epsilon}}^{m} \rightarrow$ $\bar{F}_{i, j, k, y}$ which satisfies the conclusions of A.1.1 and A.1.2 (for $p=z_{2}$ and $\left.m=\operatorname{dim} \bar{F}_{i, j, k, y}\right)$. 
Define a foliation $\hat{\mathcal{G}}_{i, j, k, y}$ on $B_{\bar{\epsilon}}^{m}$ by

$$
\hat{\mathcal{G}}_{i, j, k, y}=f_{p}^{-1}\left(\overline{\mathcal{G}}_{i, j, k, y}\right) .
$$

Let $\alpha$ denote the dimension of the leaves of $\hat{\mathcal{G}}_{i, j, k, y}$; note that $\beta=m-\alpha$. Without loss of generality we may assume that the first $\alpha$-coordinates of $\mathbf{R}^{m}$ are tangent to $\hat{\mathcal{G}}_{i, j, k, y}$ at the origin. Thus there is a smooth function $f: \mathbf{R}^{m} \rightarrow \mathbf{R}^{\beta}$ so that the following properties hold near the origin of $\mathbf{R}^{m}$. In what follows we identify $\mathbf{R}^{m}$ with $\mathbf{R}^{\alpha} \times \mathbf{R}^{\beta}$, and denote a variable point in $\mathbf{R}^{\alpha} \times \mathbf{R}^{\beta}$ by $(u, v)$.

3.3.8.1. (a) $f(0,0)=0$ and $D_{u} f(0,0)=0 ; f(0, v)=v$ for all $v \in \mathbf{R}^{\beta}$.

(b) For each $v \in \mathbf{R}^{\beta}$ define $f_{v}: \mathbf{R}^{\alpha} \rightarrow \mathbf{R}^{\beta}$ by $f_{v}(x)=f(x, v)$. The leaf of $\hat{\mathcal{G}}_{i, j, k, y}$ thru the point $(0, v)$ coincides with the graph of $f_{v}$.

We note that the curvature bounds placed on $\overline{\mathcal{G}}_{i, j, k, y}$ by 3.3.1(a) lead (thru A.1.4) to curvature bounds for $\hat{\mathcal{G}}_{i, j, k, y}$, which can be expressed in terms of the function $f(u, v)$ as follows: Let $u=\left(u_{1}, u_{2}, \ldots, u_{\alpha}\right)$ and $v=\left(v_{1}, v_{2}, \ldots, v_{\beta}\right)$ denote the standard coordinates for the variables $u$ and $v$ respectively.

3.3.8.1. (c) Near the origin of $\mathbf{R}^{\alpha} \times \mathbf{R}^{\beta}$ we have that $\left|\partial^{2} f / \partial u_{a_{1}} \partial u_{a_{2}}\right|<$ $\mathcal{O}(\epsilon) t_{i, j, 2}^{-1}$ and $\left|\partial^{2} f / \partial v_{b} \partial u_{a}\right|<\mathcal{O}(\epsilon) t_{i, j, 2}^{-1}$ for all $a_{1}, a_{2}, a \in\{1,2, \ldots, \alpha\}$ and all $b \in\{1,2, \ldots, \beta\}$.

Define a map $\hat{s}_{i, j, k, y}: \hat{B}_{i, j, k, y} \rightarrow B_{\bar{\epsilon}}^{m}$ by

$$
\hat{B}_{i, j, k, y}=s_{i, j, k, y}^{-1}\left(\operatorname{Image}\left(f_{p}\right)\right)
$$

and

$$
\hat{s}_{i, j, k, y}=f_{p}^{-1} \circ s_{i, j, k, y} .
$$

It follows from 3.0, 3.3.3, 3.3.8.1(a)-(c), and from A.1.1-A.1.3 and A.1.3.3.1 (as applied to our present $f_{p}$ and also to the exponential map used in 3.3.3(c)), and from the preceding definition, that $\hat{s}_{i, j, k, y}$ satisfies the following properties:

3.3.8.2. (a) $\left\|D \hat{s}_{i, j, k, y}-L\right\| \ll \epsilon$ for some linear distance preserving map $L: \mathbf{R}^{\beta} \rightarrow \mathbf{R}^{m}$.

(b) $\left\|D^{2} \hat{s}_{i, j, k, y}\right\| \ll B$, where $B>0$ depends only on $m$ and $A^{\prime}$ (cf. A.2.2).

(c) Let $T_{0}$ denote the tangent plane to Image $\left(\hat{s}_{i, j, k, y}\right)$ at the origin of $\mathbf{R}^{m}$; we also have the subspace $0 \times \mathbf{R}^{\beta}$ of $\mathbf{R}^{m}=\mathbf{R}^{\alpha} \times \mathbf{R}^{\beta}$. We have that

$$
\Theta\left(T_{0}, 0 \times \mathbf{R}^{\beta}\right) \ll \epsilon .
$$

Now, by appealing to Properties 3.3.8.1 and 3.3.8.2 we can define a smooth embedding $\hat{g}_{1}: V \rightarrow \mathbf{R}^{\beta}$, from a small open neighborhood $V$ of the origin in $\mathbf{R}^{\beta}$, as follows: 
3.3.8.3. (a) $\hat{g}_{1}(v)=\hat{s}_{i, j, k, y}^{-1}\left(u_{v}, f\left(u_{v}, v\right)\right)$

where $u_{v} \in \mathbf{R}^{\alpha}$ is uniquely determined by the requirement:

3.3.8.3. (b) $\left(u_{v}, f\left(u_{v}, v\right)\right) \in \operatorname{Image}\left(\hat{s}_{i, j, k, y}\right)$.

To complete the verification of Claim 3.3.8 it will suffice to show that $\hat{g}_{1}$ satisfies the following properties:

3.3.8.4. (a) $\left\|D \hat{g}_{1 \mid 0}-\hat{I}_{1}\right\| \ll \epsilon$ for some linear isometry $\hat{I}_{1}: \mathbf{R}^{\beta} \rightarrow \mathbf{R}^{\beta}$.

(b) $\left\|D^{2} \hat{g}_{1 \mid 0}\right\|<\mathcal{O}(\epsilon) t_{i, j, 2}^{-1}$.

To verify 3.3.8.4(a) we first define a map $\widetilde{g}_{1}: V \rightarrow \operatorname{Image}\left(s_{i, j, k, y}\right)$ defined by

$$
\widetilde{g}_{1}(v)=\left(u_{v}, f\left(u_{v}, v\right)\right)
$$

where $u_{v}$ is as in 3.3.8.3(b). And we let $p_{2}: \mathbf{R}^{m} \rightarrow \mathbf{R}^{\beta}$ denote the standard projection of $\mathbf{R}^{m}=\mathbf{R}^{\alpha} \times \mathbf{R}^{\beta}$ onto its second factor. Note that:

3.3.8.4.1. (a) $D\left(\widetilde{g}_{1}\right)_{\mid 0}=\left(p_{2} \mid T_{0}\right)^{-1}$,

(b) $\hat{g}_{1}=s_{i, j, k, y}^{-1} \circ \widetilde{g}_{1}$,

where $T_{0}$ comes from 3.3.8.2(c). Now Property 3.3.8.4(a) is a direct consequence of 3.3.8.4.1(a) and (b) and 3.3.8.2(a) and (c).

To verify 3.3.8.4(b) we need some more maps. For each $v \in V$ we let $u_{v} \in \mathbf{R}^{\alpha}$ be as in 3.3.8.3(b), and we define a map $h_{v}: V \rightarrow u_{v} \times \mathbf{R}^{\beta} \subset \mathbf{R}^{m}$ by

$$
h_{v}(w)=\left(u_{v}, f\left(u_{v}, w\right)\right)
$$

for all $w \in V$. Let $P_{v}$ denote the tangent plane to $\hat{\mathcal{G}}_{i, j, k, y}$ at $\left(u_{v}, f\left(u_{v}, v\right)\right)$; define a map $\rho_{v}: \mathbf{R}^{m} \rightarrow u_{v} \times \mathbf{R}^{\beta}$ by

$$
\rho_{v}(q)=\left(P_{v}+q\right) \cap\left(u_{v} \times \mathbf{R}^{\beta}\right)
$$

for all $q \in \mathbf{R}^{m}$. Let $T_{v}$ denote the tangent plane to Image $\left(\hat{s}_{i, j, k, y}\right)$ at $\left(u_{v}, f\left(u_{v}, v\right)\right)$. Note that $h_{v}$ and $\rho_{v}$ satisfy the following property:

3.3.8.4.2. (a) $D \widetilde{g}_{1 \mid v}=\left(\rho_{v} \mid T_{v}\right)^{-1} \circ\left(D h_{v \mid v}\right)$.

It also follows from Properties 3.3.8.1(a)-(c), and from 3.3.8.2(a)-(c), that:

3.3.8.4.2. (b) $\left\|D h_{v \mid v}-D h_{0 \mid 0}\right\|<\mathcal{O}(\epsilon)|v| t_{i, j, 2}^{-1}$,

(c) $\left\|D s_{i, j, k, y}^{-1} \circ\left(\rho_{v} \mid T_{v}\right)^{-1}-D s_{i, j, k, y}^{-1} \circ\left(\rho_{0} \mid T_{0}\right)^{-1}\right\| \ll \mathcal{O}(\epsilon)|v| t_{i, j, 2}^{-1}$,

(d) $\left\|D s_{i, j, k, y}^{-1} \circ\left(\rho_{v} \mid T_{v}\right)^{-1}\right\|<2$ and $\left\|h_{v}\right\|<2$ for all $v \in V$, 
where we use Euclidean parallel translation to identify the ranges of the two linear maps $D h_{v \mid v}$ and $D h_{0 \mid 0}$, and also use Euclidean parallel translation to identify the domains and ranges of the two linear maps $D s_{i, j, k, y}^{-1} \circ\left(\rho_{v} \mid T_{v}\right)^{-1}$ and $D s_{i, j, k, y}^{-1} \circ\left(\rho_{0} \mid T_{0}\right)^{-1}$. Now, by combining Properties 3.3.8.4.2(a)-(d) with 3.3.8.4.1(b), and recalling that $t_{i, j, 2} \ll \epsilon$ (cf. 3.0), we conclude that

$$
\left\|D \hat{g}_{1 \mid v}-D \hat{g}_{1 \mid 0}\right\|<\mathcal{O}(\epsilon)|v| t_{i, j, 2}^{-1},
$$

where the domains and ranges of the linear maps are identified under Euclidean parallel translation. Note that 3.3.8.4(b) is an immediate consequence of this last inequality.

3.4. $\bar{\Lambda}_{i, j, k, x} \times B_{i, j, 4, y} \rightarrow B_{i, j, 4, y}$ and the fixed point set $C_{i, j, k, y}$. It follows from 3.1.5(a) and (b), and from the fact that the leaves of the foliation $\mathcal{G}_{i, j, k, x}$ are just the orbits of the action $G_{t_{i}, x} \times F_{i, j, k, y} \rightarrow F_{i, j, k, y}$, that the leaves of $\mathcal{G}_{i, j, k, x}$ are permuted by the action $\Lambda_{i, j, k, x} \times F_{i, j, k, y} \rightarrow F_{i, j, k, y}$ for all $4 \leq k \leq(m+4)^{2}-1$. It follows from 3.1.5(c), and from the definitions given for $\bar{F}_{i, j, k, y}, \overline{\mathcal{G}}_{i, j, k, y}, \bar{\Lambda}_{i, j, k, x}$ in 3.3 , that the action $\Lambda_{i, j, k, x} \times F_{i, j, k, y} \rightarrow F_{i, j, k, y}$ induces an isometric action

$$
\bar{\Lambda}_{i, j, k, x} \times \bar{F}_{i, j, k, y} \rightarrow \bar{F}_{i, j, k, y}
$$

which permutes the leaves of $\overline{\mathcal{G}}_{i, j, k, y}$. Now since $B_{i, j, k, y}$ is obtained from $\bar{F}_{i, j, k, y}$ by collapsing each leaf of $\overline{\mathcal{G}}_{i, j, k, y}$ to a point we also have the action

$$
\bar{\Lambda}_{i, j, k, x} \times B_{i, j, k, y} \rightarrow B_{i, j, k, y},
$$

for each $4 \leq k \leq(m+4)^{2}-1$. Note that $B_{i, j, k, y}$ is an open subset of $B_{i, j, 4, y}$ (cf. 3.3.4(b), and recall that $F_{i, j, k, y}$ is an open subset of $F_{i, j, x}$ for all $k$ and $\left.\mathcal{G}_{i, j, k, y}=\mathcal{G}_{i, j, 4, y} \mid F_{i, j, k, y}\right)$. Note also that $\bar{\Lambda}_{i, j, k, x} \subset \bar{\Lambda}_{i, j, 4, x}$ for all $k \geq 4$ (cf. 3.1.1, 3.1.4, 3.1.5(c) and (d)). Thus the action of $\bar{\Lambda}_{i, j, k, x}$ on $B_{i, j, k, y}$ extends to an action

$$
\bar{\Lambda}_{i, j, k, x} \times B_{i, j, 4, y} \rightarrow B_{i, j, 4, y} .
$$

This last action is not an isometric action, but it is nearly so. In fact the following properties can be deduced from 3.3.6(a) and (b):

3.4.1. For any $g \in \bar{\Lambda}_{i, j, k, x}$ and any $z \in B_{i, j, 4, y}$ there is a linear isometry $L_{z}: \mathbf{R}^{\beta} \rightarrow \mathbf{R}^{\beta}$ (where $\mathbf{R}^{\beta}$ comes from 3.3.6) which satisfies:

(a) $\left|D g_{\mid z}-L_{z}\right| \ll \epsilon$;

(b) $\left|D^{2} g\right|<\mathcal{O}(\epsilon) t_{i, j, 2}^{-1}$.

Note that the following additional property can be deduced from 3.3.5, and from the fact that the action of $g$ on $B_{i, j, 4, y}$ leaves invariant the subset $B_{i, j, k, y} \subset B_{i, j, 4, y}$ : 
3.4.1. (c) $|g(0)| \ll t_{i, j, k-1, l}$ for $l=(m+4)^{2}-1$.

We denote by $C_{i, j, k, y} \subset B_{i, j, 4, y}$ the fixed point set of the action $\bar{\Lambda}_{i, j, k, x} \times$ $B_{i, j, 4, y} \rightarrow B_{i, j, 4, y}$. We will verify the following important properties of $C_{i, j, k, y}$ for all $5 \leq k \leq(m+4)^{2}-1$ :

3.4.2. (a) There is $q \in C_{i, j, k, y}$ satisfying $|q| \ll t_{i, j, k-1, l}$ for $l=(m+4)^{2}-1$.

(b) $K\left(C_{i, j, k, y} ; \mathbf{R}^{\beta}\right) \ll \mathcal{O}(\epsilon) t_{i, j, 2}^{-1}$, where $K\left(C_{i, j, k, y} ; \mathbf{R}^{\beta}\right)$ is defined to be the curvature $K(r ; M)$ of 0.1 when in 0.1 we set $M=\mathbf{R}^{\beta}$ and we let $r: C_{i, j, k, y} \rightarrow\{1\}$ denote the constant map.

(c) $C_{i, j, k, y}$ is a closed subset of $B_{i, j, 4, y}$.

We define the point $q$ of 3.4 .2 (a) by $q=\operatorname{limit}_{n \rightarrow \infty} q_{n}$ where the sequence $q_{1}, q_{2}, q_{3}, \ldots$ is defined inductively as follows:

$$
q_{1}=0 ; \quad q_{n+1}=\left|\bar{\Lambda}_{i, j, k, x}\right|^{-1}\left(\sum_{g \in \bar{\Lambda}_{i, j, k, x}} g\left(q_{n}\right)\right) .
$$

It follows from 3.4.1(a) and (c) that $q$ is well-defined and satisfies 3.4.2(a). We note that 3.4.2(b) is a direct consequence of 3.4.1(a) and (b). Property 3.4.2(c) is immediate. We leave as an exercise for the reader to verify the following additional property for $C_{i, j, k, y}$ for all $5 \leq k \leq(m+4)^{2}-1$. Let $B\left(t_{i, j, 4}\right)$ denote the open ball of radius $t_{i, j, 4}$ centered at the origin of $B_{i, j, 4, y}$ (cf. 3.3.5).

3.4.2. (d) $C_{i, j, k, y} \cap B\left(t_{i, j, 4}\right)$ is a path connected manifold.

Note that for any $5 \leq k<(m+4)^{2}-1$ we have $\bar{\Lambda}_{i, j, k+1, x} \subset \bar{\Lambda}_{i, j, k, x}$ by 3.1.1, 3.1.4, 3.1.5(c) and (d), and 3.3; thus we have that

$$
C_{i, j, k, y} \subset C_{i, j, k+1, y} .
$$

Now we may use an induction argument (induction over $\operatorname{dim}\left(C_{i, j, k, y}\right)$ ), in conjunction with the preceding inclusion and with Properties 3.4.2(c) and (d), to conclude that for some $5 \leq k<(m+4)^{2}-2$ we have that:

3.4.3. $C_{i, j, k, y} \cap B\left(t_{i, j, 4}\right)=C_{i, j, k+1, y} \cap B\left(t_{i, j, 4}\right)=C_{i, j, k+2, y} \cap B\left(t_{i, j, 4}\right)$.

We also have as a consequence of 3.4.3 that:

3.4.4. $\bar{\Lambda}_{i, j, k, x}=\bar{\Lambda}_{i, j, k+1, x}$

for $k$ as in 3.4.3. To verify 3.4.4 we use 3.4.2(a) (with $k$ replaced by $k+2$ ) to choose $q \in C_{i, j, k+2, y}$ which satisfies:

3.4.5. (a) $|q| \ll t_{i, j, k+1, l}$ for $l=(m+4)^{2}-1$. 
It follows from 3.4.3 that:

3.4.5. (b) $q \in C_{i, j, k, y}$.

Note it also follows from 3.3.1(b) (with $k$ replaced by $k+1$ in 3.3.1(b)), 3.3.3, 3.3.4(a), and from 3.4.5(b) and from the fact that $\pi_{i, j, k, y}^{-1}(q)$ is a covering space for $\pi_{i, j, k, y}^{-1}(q) / \bar{\Lambda}_{i, j, k, x}$, that the following property holds:

3.4.5. (c) diameter $\left(\pi_{i, j, k, y}^{-1}(q) / \bar{\Lambda}_{i, j, k, x}\right)<2 \tau t_{i, j, k+2}$.

Let $L \in \mathcal{G}_{i, j, k, y}$ denote the leaf which covers the leaf $\pi_{i, j, k, y}^{-1}(q) \in \overline{\mathcal{G}}_{i, j, k, y}$, then we may reinterpret Properties 3.4.5(a) and (c) as follows:

3.4.5. (d) There is $\hat{q} \in L$ with $d_{\hat{V}}(y, \hat{q}) \ll t_{i, j, k+1, l}$ for $l=(m+4)^{2}-1$.

(e) $L$ is left invariant by the action $\Lambda_{i, j, k, x} \times \hat{V} \rightarrow \hat{V}$. Moreover there is a generating set $\left\{s_{1}, s_{2}, \ldots, s_{t}\right\}$ for $\Lambda_{i, j, k, x}$ such that for all $s_{r}$ we have

$$
d_{\hat{V}}\left(\hat{q}, s_{r}(\hat{q})\right)<8 \tau t_{i, j, k+2} .
$$

Now 3.4.4 follows from 3.4.5(d) and (e), and from 3.0, 3.1.4, 3.2.1(a).

3.5. The infranil core $r: U \rightarrow B$. In this subsection we shall complete the proof of Theorem 0.3 by defining $r: U \rightarrow B$ and $\delta_{c}>0$ of 0.3 which satisfy Properties 0.3(a)-(c).

First we define $\delta_{c}$ by:

3.5.1. (a) $\delta_{c}=t_{i, j, k, 3}$,

where for the duration of this subsection the positive integers $i, j, k$ are as in 3.1.1, 3.1.3, 3.4.3 and 3.4.4, respectively. Now we let $q \in C_{i, j, k+1, y}$ be as in 3.4.5(a). Recall that $C_{i, j, k, y} \subset \mathbf{R}^{\beta}$; let $P \subset \mathbf{R}^{\beta}$ denote the plane which is tangent to $C_{i, j, k, y}$ at $q$; and let $\mathcal{P}: \mathbf{R}^{\beta} \rightarrow P$ denote the orthogonal projection onto $P$. Set:

3.5.1. (b) $B=\left\{u \in P:|u-q|<\delta_{c}\right\}$;

then $B$ is isometrically equivalent to an open ball of radius $\delta_{c}$ centered at the origin (i.e., at $q$ ) in some Euclidean space (i.e., in $P$ ), as is required in 0.2.1(b) and 0.3(a). Note that $\left(\mathcal{P} \mid C_{i, j, k, y}\right)^{-1}$ is well-defined (cf. 3.4.2(b)(d)) and maps $B$ diffeomorphically onto an open subset $\widetilde{B} \subset C_{i, j, k, y}$. Define the map $r: U \rightarrow B$ by:

3.5.1. (c) $U=\pi_{i, j, k, y}^{-1}(\widetilde{B}) / \bar{\Lambda}_{i, j, k, x}$.

(d) Note that $\pi_{i, j, k, y}$ induces a map $\widetilde{\pi}_{i, j, k, y}: U \rightarrow \widetilde{B}$; let $r$ denote the composition of $\widetilde{\pi}_{i, j, k, y}$ with $\mathcal{P} \mid \widetilde{B}$. 
First we must verify that $r: U \rightarrow B$ is an infranil core for $M$ as defined in 0.2 . We note that for each $z \in B$ there is a leaf $L_{z} \in \mathcal{G}_{i, j, k, y}$ which is left invariant by the action $\Lambda_{i, j, k, x} \times \hat{V} \rightarrow \hat{V}$ such that $r^{-1}(z)=L_{z} / \Lambda_{i, j, k, x}$. Since the leaves of $\mathcal{G}_{i, j, k, y}$ are just the orbits of the free action $G_{i, j, x} \times$ $F_{i, j, k, y} \rightarrow F_{i, j, k, y}$, it follows that each fiber $r^{-1}(z)$ is diffeomorphic to the infranil manifold $G_{i, j, x} / \Lambda_{i, j, k, x}$; cf. 3.1.5 and 3.2. This shows that $r$ satisfies 0.2.1(a). We note that 0.2.1(b) is immediate from 3.5.1(b). To see that $r$ satisfies 0.2.1(c) we argue as follows. First we deduce from 3.4.2(b) and 3.3.1(a) that:

3.5.2. (a) $K(\mathcal{G} ; U)<\mathcal{O}(\epsilon) t_{i, j, 2}^{-1}$

where $\mathcal{G}$ denotes the foliation for $U$ by the fibers of $r$. And we may deduce from 3.4.5(c) and 3.5.1(a) (see also 3.0) that:

3.5.2. (b) diameter $(U)<\mathcal{O}\left(\delta_{c}\right)$.

Let $\hat{q} \in \hat{V}$ denote a point in the preimage of $r^{-1}(q \sim 0)$ under the covering space projection $\pi: \hat{V} \rightarrow V$ (cf. Appendix 2); and set:

3.5.2. (c) $\hat{U}=\pi^{-1}(U) \cap B(\hat{q}, t)$,

where $t$ denotes the $\mathcal{O}\left(\delta_{c}\right)$ of 3.5.2(b) and where $B(\hat{q}, t)$ denotes the open ball of radius $t$ centered at $\hat{q}$ in $\hat{V}$. Note that 3.4.2(b) and 3.3.1(a) (together with the fact that $F_{i, j, k, y}$ is locally a totally geodesic submanifold of $\hat{V}$ ) also imply:

3.5.2. (d) $K(\hat{U} ; \hat{V})<\mathcal{O}(\epsilon) t_{i, j, 2}^{-1}$,

where $K(\hat{U} ; \hat{V})$ denotes the curvature of the constant map $\hat{U} \rightarrow\{1\}$ (as described in 0.1) for the submanifold $\hat{U} \subset \hat{V}$ with respect to the Riemannian metric $g_{\hat{V}}^{\prime}$ of Appendix 2. We deduce from 3.5.2(c) and (d), and from A.2.1(b) and 3.0 and 3.5.1(a), that the following holds for $\hat{U} \subset \hat{V}$ :

3.5.2. (e) $\hat{U}$ has a well-defined tubular neighborhood of radius $\delta_{c}$ in $\hat{V}$.

Finally, we may deduce that $U$ satisfies Property 0.2.1(c) from 3.5.2(b), (c) and $(\mathrm{e})$, and from 3.0, 3.1.1, 3.1.3, 3.1.4, 3.1.5(c) and (d), 3.4.4.

Now we must verify that $r: U \rightarrow B$ satisfies Properties 0.3(a)-(c).

Towards verifying $0.3(\mathrm{a})$ we first note that $B$ has radius equal $\delta_{c}$ (cf. 3.5.1(b)). Moreover $c \in(0, \eta)$ by 3.0 and 3.5.1(a). (Recall that we are proving 0.3 for the special case $n=0$.) This completes the verification of $0.3(\mathrm{a})$.

Next we will verify $0.3(\mathrm{c})$. Let $\hat{q} \in \hat{V}$ be as in 3.4.5(d), and define $p^{\prime} \in U$ of 0.3 to be the image of $\hat{q}$ under the covering projection $\pi: \hat{V} \rightarrow V$. Note that $\hat{q}$ of 3.5.3 may be chosen equal to $\hat{q}$ of 3.5.2(c); thus we have: 
3.5.3. (a) $\left|r\left(p^{\prime}\right)\right|=0$.

[Actually we have $r\left(p^{\prime}\right)=q \in P$; but we are identifying $(P, q)$ isometrically with $\left(\mathbf{R}^{n}, 0\right)$ for some integer $n$, in order that $B$ be an open ball centered at the origin of $\mathbf{R}^{n}$ as is required in 0.2.1.] Note also that it follows from 3.0, 3.2.1(a), 3.4.5(d), 3.5.1(a), and from the fact that $x \in E$ is mapped to $p \in M$ under the composition $E \stackrel{\rho}{\longrightarrow} \hat{V} \stackrel{\pi}{\longrightarrow} V$ (cf. 3.1), that:

3.5.3. (b) $d_{M}\left(p, p^{\prime}\right)<\epsilon \delta_{c}$,

where $d_{M}($,$) denotes the distance in the manifold M$ of 0.3 . Now $0.3(\mathrm{c})$ is a consequence of 3.5.3(a) and (b).

It remains to verify Property $0.3(\mathrm{~b})$; i.e., we must find a number $\theta \in(0,1)$ which depends only on $\operatorname{dim} M$ such that Properties 0.2.2(a)-(d) hold, for $\delta=\delta_{c}$ in 0.2 .2 .

Note that Property 0.2 .2 (c) can be deduced fairly directly from 3.0, 3.3.1(a), 3.3.3(a), and 3.5.1(a)-(d).

Towards verifying $0.2 .2(\mathrm{~b})$ we first note that

$$
r^{-1}(0)=\pi_{i, j, k, y}^{-1}(q) / \bar{\Lambda}_{i, j, k, x} ;
$$

thus we may deduce from 3.0, 3.5.1(a), and from 3.4.5(c) that

$$
\operatorname{diameter}\left(r^{-1}(0)\right) \ll \epsilon \delta_{c} .
$$

Now 0.2.2(b) follows from this last inequality and from Properties 3.5.2(a) and $0.2 .2(\mathrm{c})$.

Next we verify $0.2 .2(\mathrm{a})$. We have already verified part of this property by noting above that 3.4.2(b) and 3.3.1(a) together imply 3.5.2(a) and (d). Unfortunately the curvature bounds for $\mathcal{G}$ provided by 3.5.2(a) can not be translated into curvature bounds for $r$. [Although the converse is true: A bound on $K(r ; M)$ can be translated into a bound for $K(\mathcal{G} ; U)$ and for $K(\hat{U}, \hat{V})$.] What we need is a stronger version of 3.3.1(a) which we can combine with 3.4.2(b) to deduce 0.2.2(a), and this stronger version is provided by the following claim. Let $\pi_{i, j, k, y}: \bar{F}_{i, j, k, y} \rightarrow B_{i, j, k, y}$ denote the fiber bundle projection defined in 3.3, and $\bar{V}$ be as in 3.3.1(a).

Claim 3.5.4. $K\left(\pi_{i, j, k, y} ; \bar{V}\right)<\mathcal{O}(\epsilon) t_{i, j, 2}^{-1}$.

The verification of this claim, which will be carried out at the end of this chapter, relies on 3.3.1(a) and 3.3.6. Note that 3.5.4 and 3.4.2(b), together with 3.3.3 and 3.5.1, imply that

$$
K(r ; M)<\mathcal{O}(\epsilon) t_{i, j, 2}^{-1} .
$$

Now 0.2.2(a) follows immediately from this last inequality and from 3.0, 3.5.1(a). 
Finally we will verify $0.2 .2(\mathrm{~d})$. Let $\left.v \in T M\right|_{U}$ be perpendicular to $U$; and denote by $\hat{v} \in T \hat{V}$ a vector which is in the pre-image of under the covering projection $\pi: \hat{V} \rightarrow V$. In order to verify that $v$ satisfies the conclusions of 0.2.2(d) it will suffice to consider the following special cases:

(i) The foot of $\hat{v}$ lies in $F_{i, j, k, y}$, and $\hat{v}$ is perpendicular to $F_{i, j, 4, y}$ in $\hat{V}$;

(ii) the foot of $\hat{v}$ lies in $F_{i, j, k, y}$, and $\hat{v}$ is tangent to $F_{i, j, 4, y}$.

If $\hat{v}$ is perpendicular to $F_{i, j, 4, y}$ in $\hat{V}$ then we argue as follows: Since $F_{i, j, 4, y}$ is an open subset of the fixed point set for the compact group action $C_{i, j, x} \times$ $\hat{V} \rightarrow \hat{V}$ (cf. 3.2), we can choose $g \in C_{i, j, x}$ so that:

3.5.5. (a) $\lambda_{1}<\Theta(\hat{v}, g(\hat{v}))$

where $\lambda_{1}>0$ depends only on $\operatorname{dim} \hat{V}$. Recall that $\mathcal{G}_{i, x}$ is the foliation of $E$ by the orbits of the free action $G_{t_{i}, x} \times E \rightarrow E$; let $u$ denote the foot of $\hat{v}$, let $\hat{u}$ denote a point in the pre-image of $u$ under the map $\rho: E \rightarrow \hat{V}$, and let $\hat{L}$ denote the leaf of $\mathcal{G}_{i, x}$ which contains the point $\hat{u}$. We have shown in 3.2.13(d) that:

3.5.5. (b) diameter $\left(\hat{L} / \Lambda_{t_{i, j, k}, x}^{\prime}\right)<2 \tau t_{i+1}$.

[Note that there is no loss of generality in choosing the preimage $\hat{u}$ of $u$ so that our present choice of $\hat{L}$ is equal the $\hat{L}$ of 3.2.13.] Thus, for some $g^{\prime} \in \Lambda_{t_{i, j, k+1}, x}^{\prime}$ (cf. 3.1.5(d)), we have that:

3.5.5. (c) $d_{\hat{L}}^{\prime}\left(g(\hat{u}), g^{\prime}(\hat{u})\right)<\lambda_{2} t_{i+1}$

where $\lambda_{2}>1$ depends only on $\operatorname{dim} E$. Since $g(u)=u$, and since $\rho: E \rightarrow \hat{V}$ is a Riemannian submersion, we may deduce from 3.5.5(c) that:

3.5.5. (d) $d_{L}^{\prime}\left(u, g^{\prime}(u)\right)<\lambda_{2} t_{i+1}$,

where the leaf $L \in \mathcal{G}_{i, j, k, y}$ contains $u$. Hence we can find a smooth path $\hat{f}:[0,1] \rightarrow L$ which satisfies the following property:

3.5.5. (e) $\hat{f}(0)=u$ and $\hat{f}(1)=g^{\prime}(u)$; length $(\hat{f})<2 \lambda_{2} t_{i+1}$.

Now the desired path $f:[0,1] \rightarrow U$ in $0.2 .2(\mathrm{~d})$ may be defined to be the composition of $\hat{f}$ with the covering projection $\pi: \hat{V} \rightarrow V$. That $f$ satisfies the conclusions of $0.2 .2(\mathrm{~d})$ can be deduced from 3.0, 3.5.1(a), and 3.5.5(a), (c) and (e). This completes the verification of $0.2 .2(\mathrm{~d})$ for the case when $\hat{v}$ is perpendicular to $F_{i, j, 4, y}$ in $\hat{V}$.

If $\hat{v}$ is tangent to $F_{i, j, 4, y}$ in $\hat{V}$ then we verify $0.2 .2(\mathrm{~d})$ as follows: Let $\bar{v}$ denote the image of $\hat{v}$ under the composition of the covering map $F_{i, j, 4, y} \rightarrow$ $\bar{F}_{i, j, 4, y}$ (recall that $\bar{F}_{i, j, 4, y}=F_{i, j, 4, y} / \Lambda_{i, j, 4, x}^{\prime}$ ) with the projection map $\pi_{i, j, k, y}$ : 
$\bar{F}_{i, j, 4, y} \rightarrow B_{i, j, 4, y}$. It follows from 3.0, 3.3.1, 3.3.3, 3.5.1, and from the fact that $v$ is perpendicular to $U$ in $\bar{F}_{i, j, 4, x} / \bar{\Lambda}_{i, j, k, x}$, that:

3.5.6. (a) $\Theta\left(\bar{v}, C_{i, j, k, x}\right)>\pi / 2-\mathcal{O}(\epsilon)$,

where $C_{i, j, k, x}$ is the fixed point set of the action $\bar{\Lambda}_{i, j, k, x} \times B_{i, j, 4, x} \rightarrow B_{i, j, 4, x}$. Since the foot of $\bar{v}$ (denoted by $\bar{u}$ ) is in $C_{i, j, k, x}$, it follows from 3.5.6(a) (see also 3.4.1) that we can choose $\bar{g} \in \bar{\Lambda}_{i, j, k, x}$ such that:

3.5.6. (b) $\Theta(\bar{v}, \bar{g}(\bar{v}))>\lambda_{3}$

where $\lambda_{3}$ depends only on $\operatorname{dim} B_{i, j, k, y}$. Note that it follows from 3.3.1(b), 3.4.3, 3.4.4, and from 3.5.1 that the fiber $r^{-1}(\bar{u})$ has a finite covering space $Y \rightarrow r^{-1}(\bar{u})$ which satisfies:

3.5.6. (c) diameter $(Y)<2 \tau t_{i, j, k+1}$;

(d) $Y / \bar{\Lambda}_{i, j, k, x}=r^{-1}(\bar{u})$.

Let $u \in Y / \bar{\Lambda}_{i, j, k, x}$ denote the foot of $v$ and let $u^{\prime} \in Y$ denote a pre-image of $u$ under the covering map $Y \rightarrow r^{-1}(\bar{u})$. Using 3.5.6(c) and (d) we may choose a path $\hat{f}:[0,1] \rightarrow Y$ which satisfies:

3.5.6. (e) $\hat{f}(0)=u^{\prime}$ and $\hat{f}(1)=\bar{g}\left(u^{\prime}\right)$; length $(\hat{f})<2 \tau t_{i, j, k+1}$.

Now the desired path $f:[0,1] \rightarrow U$ of $0.2 .2(\mathrm{~d})$ may be defined to be the composition of $\hat{f}$ with the covering projection $Y \rightarrow r^{-1}(\bar{u})$. That $f$ and $v$ satisfy the conclusions of 0.2.2(d) follows from 3.0, 3.5.1(a), 3.5.4, 3.3.6(a), and 3.5.6(b) and (e). This completes the verification for $0.2 .2(\mathrm{~d})$ when $\hat{v}$ is tangent to $F_{i, j, 4, y}$.

\section{Verification of Claim 3.5.4.}

Since $\bar{F}_{i, j, k, y}$ is an open subset of a totally geodesic submanifold of $\bar{V}$, it will suffice to show that there is a positive number $\kappa$, with $\kappa<\mathcal{O}(\epsilon) t_{i, j, 2}^{-1}$, such that for any smooth path $h:[0,1] \rightarrow \bar{F}_{i, j, k, y}$ we have:

3.5.4.1. $\left\|P_{h}\left(D \pi_{i, j, k, y \mid h(0)}\right), D \pi_{i, j, k, y \mid h(1)}\right\|<\kappa($ length $(h))$.

Recall (cf. 0.1) that for any $v \in T\left(\bar{F}_{i, j, k, y}\right)_{\mid h(1)}$ we have that

$$
P_{h}\left(D \pi_{i, j, k, y \mid h(0)}\right)(v)=P \circ D \pi_{i, j, k, y \mid h(0)} \circ \rho \circ P_{\bar{h}}(v)
$$

where $P$ is Euclidean parallel translation in $B_{i, j, k, y}$, and $\rho: T \bar{V}_{\mid h(0)} \rightarrow$ $T\left(\bar{F}_{i, j, k, y}\right)_{\mid h(0)}$ denotes orthogonal projection, and $P_{\bar{h}}$ is parallel translation along $\bar{h}$ in $\bar{V}$ where $\bar{h}(t)=h(1-t)$ for all $t \in[0,1]$.

We will first verify 3.5.4.1 in the following two special cases: 
Case I. We suppose in this case that $h$ of 3.5.4.1 satisfies Image $(h) \subset L$ for some $L \in \overline{\mathcal{G}}_{i, j, k, y}$; and we shall deduce 3.5.4.1 from 3.3.1(a). In more detail, we may proceed as in the verification for Claim 3.3.8 given above. Set $p=h(0)$ and let $f_{p}: B_{\bar{\epsilon}}^{m} \rightarrow \bar{F}_{i, j, k, y}$ be a smooth embedding as in A.1.1 and A.1.2. Let $\hat{\mathcal{G}}_{i, j, k, y}$ and $f: \mathbf{R}^{m} \rightarrow \mathbf{R}^{\beta}$ be as in 3.3.8.1. There will be no loss of generality in assuming that Image $(h) \subset$ Image $\left(f_{p}\right)$; because if 3.5.4.1 holds for all curves of sufficiently small length, then it must hold for all curves. Thus we define $\hat{h}:[0,1] \rightarrow B_{\bar{\epsilon}}^{m}$ by

$$
\hat{h}=f_{p}^{-1} \circ h \text {. }
$$

Recall that 3.3.8.1(c) is just a reformulation for the curvature bounds given in 3.3.1(a). Define a map $\hat{\pi}_{i, j, k, y}: B_{\bar{\epsilon}}^{m} \rightarrow B_{i, j, k, y}$ by

$$
\hat{\pi}_{i, j, k, y}=\pi_{i, j, k, y} \circ f_{p} .
$$

Note that $\hat{\pi}_{i, j, k, y}$ is equal to the composition of the orthogonal projection of $B_{\bar{\epsilon}}^{m}$ onto $\mathbf{R}^{\beta}$ (cf. 3.3.8.1) with one of the maps $s=\pi_{i, j, k, y} \circ s_{i, j, k, y}^{\prime}$ of 3.3.6. Thus we may apply 3.3.8.1(a)-(c) and 3.3.6(a) and (b) to deduce that:

3.5.4.2. $\left\|P_{\hat{h}}\left(D \hat{\pi}_{i, j, k, y \mid \hat{h}(0)}\right), D \hat{\pi}_{i, j, k, y \mid \hat{h}(1)}\right\|<\hat{\kappa}(\operatorname{length}(\hat{h}))$

for some positive number $\hat{\kappa}$ satisfying $\hat{\kappa}<\mathcal{O}(\epsilon) t_{i, j, 2}^{-1}$, where the $\Theta($,$) and P_{\hat{\alpha}}$ and length $(\hat{h})$ are all geometric constructions and measurements made with respect to the Euclidean metrics on $B_{\bar{\epsilon}}^{m}$ and on $B_{i, j, k, y}$. Let $\delta_{j}^{i} d x_{i} d x_{j}$ denote the Euclidean metric on $B_{\bar{\epsilon}}^{m}$, and let $g_{i, j} d x_{i} d x_{j}$ denote the metric $B_{\bar{\epsilon}}^{m}$ pulled back along $f_{p}$ from $\bar{V}$ (cf. A.1.1). Now, by comparing parallel translation in $B_{\bar{\epsilon}}^{m}$ with respect to these two Riemannian metrics (see Appendix 1), we see that 3.5.4.2 implies 3.5.4.1.

Case II. We suppose in this case that $h$ of 3.5.4.1 satisfies Image $(h) \subset$ Image $\left(s_{i, j, k, y}^{\prime}\right)$ for some map $s_{i, j, k, y}^{\prime}: B_{i, j, k, y}^{\prime} \rightarrow \bar{F}_{i, j, k, y}$ as in 3.3.6; and we shall deduce 3.5.4.1 from 3.3.1(a) and 3.3.6. Recall (cf. 3.3) that $s_{i, j, k, y}^{\prime}$ is a composition

$$
B_{i, j, k, y}^{\prime} \subset \mathbf{R}^{\beta} \stackrel{f_{W^{\prime}}}{\longrightarrow} W^{\prime} \stackrel{\exp }{\longrightarrow} \bar{V},
$$

where $B_{i, j, k, y}^{\prime}$ is an open subset of the Euclidean space $\mathbf{R}^{\beta}, f_{W^{\prime}}$ is a linear isometry onto a subspace of $T\left(\bar{F}_{i, j, k, y}\right)_{\mid \bar{y}^{\prime}}$ for some $\bar{y}^{\prime} \in \bar{F}_{i, j, k, y}$, and exp denotes the exponential map. Thus the Euclidean metric $\delta_{j}^{i} d x_{i} d x_{j}$ on $B_{i, j, k, y}^{\prime}$ is a very close approximation to the Riemannian metric $g_{i, j} d x_{i} d x_{j}$ gotten by pulling the Riemannian metric on $\bar{V}$ back along $s_{i, j, k, y}^{\prime}$ (see Appendix 1). In light of these few preceding remarks we may deduce from Properties 3.3.6(a) and (b) that: 
3.5.4.3. $\left|P_{h}\left(D \pi_{i, j, k, y \mid h(0)}\right)(v), D \pi_{i, j, k, y \mid h(1)}(v)\right|<\kappa^{\prime}($ length $(h))$

for any $v \in T\left(\text { image }\left(s_{i, j, k, y}^{\prime}\right)\right)_{\mid h(1)}$, where $\kappa^{\prime}$ is a positive number satisfying $\kappa^{\prime}<\mathcal{O}(\epsilon) t_{i, j, 2}^{-1}$. Finally we may deduce 3.5.4.1 from 3.5.4.3 and 3.3.1(a).

To verify 3.5.4.1 in general we note that any smooth path $h:[0,1] \rightarrow$ $\bar{F}_{i, j, k, y}$ may be point wise approximated by a piecewise smooth path $g$ : $[0,1] \rightarrow \bar{F}_{i, j, k, y}$ which satisfies:

(i) $g(0)=h(0)$ and $g(1)=h(1)$;

(ii) every smooth piece of $g$ is either a path as in Case I (reparametrized) or a path as in Case II (reparametrized);

(iii) $(1 / 3)$ length $(h)<\operatorname{length}(g)<3(\operatorname{length}(h))$.

Then, since 3.5.4.1 has been verified for Cases I and II, it follows from (i) above that 3.5.4.1 holds also for $g$. Also, since $g$ point wise approximates $h$, we have that parallel translation along $g$ approximates parallel translation along $h$. Thus, by (iii) and the two preceding remarks, we may conclude that $h$ also satisfies 3.5.4.1.

\section{Appendix 1.}

Let $(M, g)$ denote a complete $A$-regular Riemannian manifold. In this appendix we reformulate the $A$-regular condition for $(M, g)$ in terms of the local coordinates associated with the exponential map exp :TM $\rightarrow M$ (the "normal" coordinates for $M$ ). We analyze to what extent the metric $g$ is approximated by the Euclidean metric for a normal coordinate system (cf. A.1.1 and A.1.2), and to what extent germs of isometries of $M$ are approximated by linear isometries for a normal coordinate system (cf. A.1.3). We also reexamine here the notion of "curvature" for a map (cf. 0.1): We analyze the relations between curvatures computed in $(M, g)$ and curvatures computed with respect to the Euclidean metric for a normal coordinate system for $M$ (cf. A.1.4).

All of the results A.1.1-A.1.4 are referred to in the proof for Theorem 0.5 given above.

We begin by paraphrasing a result of J. Jost and H. Karcher [14, Satz 3.4 and 5.1], and of J. Bemelmans, M. Min-Oo, and E. Ruh [1]. In the next theorem we let $B_{\epsilon}^{m}$ denote the open ball of radius $\epsilon>0$ centered at the origin of $\mathbf{R}^{m}$, and we let $\partial / \partial x_{i}: \mathbf{R}^{m} \rightarrow T \mathbf{R}^{m}, i=1,2, \ldots, m$, denote the vector fields associated to the standard coordinates $x_{1}, x_{2}, \ldots, x_{m}$ for the $\mathbf{R}^{m}$.

Theorem A.1.1. There is $\eta>0$ and a collection $B=\left\{B_{i}\right\}$ of positive numbers $B_{1}, B_{2}, B_{3}, \ldots$ which depend only $A=\left\{A_{i}\right\}$ and $m=\operatorname{dim} M$. For each $p \in M$ and each $\epsilon \in(0, \eta)$ there is a smooth immersion $f: B_{\epsilon}^{m} \rightarrow M$ 
which satisfies the following properties. Let $g_{i, j}: B_{\epsilon}^{m} \rightarrow \mathbf{R}$ be defined by $g_{i, j}(x)=g\left(D f\left(\partial / \partial x_{i}(x)\right), D f\left(\partial / \partial x_{j}(x)\right)\right)$ for all $x \in B_{\epsilon}^{m}$.

(a) $f(0)=p$ and $g_{i, j}(0)=\delta_{j}^{i}$.

(b) $\left|\partial^{k} g_{i, j} / \partial x_{s_{1}} \partial x_{s_{2}} \ldots \partial x_{s_{k}}(x)\right| \leq B_{k}$ for all $k, i, j,\left\{s_{1}, \ldots, s_{k}\right\}, x \in B_{\epsilon}^{m}$.

Remark A.1.1.1. Actually Jost and Karcher [14] verify this theorem under the added hypothesis that there is a lower bound $\tau>0$ to the injectivity radius of $M$, and $\epsilon>0$ is also dependent on $\tau$. The following simple trick can be used to remove this hypothesis (cf. [1]). Since the curvature of $M$ is pinched, there is $\alpha>0$, which depends only on $\operatorname{dim} M$ and the bounds of the curvature of $(M, g)$, so that the following is true: For any $p \in M$ the exponential map exp : $B_{\alpha} \rightarrow M$ is an immersion, where $B_{\alpha}$ denotes the open ball of radius $\alpha$ centered at the origin in $T M_{p}$; moreover the $g^{*}$-injectivity radius at each point $p \in B_{\alpha / 2}$ is greater than $\tau>0$, where $g^{*}$ is the pull back along exp of the metric $g$ on $M$, and where $\tau>0$ depends only on $\operatorname{dim}$ $M$ and the curvature bounds for $(M, g)$ (cf. Cheeger and Ebin [3]). Thus the results of [14, Satz 3.4 and 5.1] may be applied to $\left(B_{\alpha}, g^{*}\right)$ near the origin of $B_{\alpha}$ to obtain a desirable coordinate system $h: B_{\epsilon}^{m} \rightarrow B_{\alpha}$ near the origin in $\left(B_{\alpha}, g^{*}\right)$; now the immersion $f: B_{\epsilon}^{m} \rightarrow M$ of Theorem A.1.2 can be defined to be the composition $B_{\epsilon}^{m} \stackrel{h}{\longrightarrow} B_{\alpha} \stackrel{\exp }{\longrightarrow} M$.

The maps $h: B_{\epsilon}^{m} \rightarrow B_{\alpha}$ referred to in the preceding remark are the harmonic coordinates for $\left(B_{\alpha}, g^{*}\right)$ near the origin (cf. [14, Satz 3.4 and 5.1]). So, roughly speaking, the maps $f: B_{\epsilon}^{m} \rightarrow M$ which Theorem A.1.1 provides are the "immersed" harmonic coordinates. The following corollary states Theorem A.1.1 is also true for the "immersed" normal coordinates. Both Corollaries A.1.2 and A.1.3 are proven below.

Corollary A.1.2. We may assume in A.1.1 that $f=\exp \circ L$, where $L$ : $\mathbf{R}^{m} \rightarrow T M_{p}$ is a linear isometry and where $\exp : T M_{p} \rightarrow M$ denotes the exponential map.

Corollary A.1.3. For given $p, p^{\prime} \in M$ we let $f: B_{\epsilon}^{m} \rightarrow M$ and $f^{\prime}: B_{\epsilon}^{m} \rightarrow$ $M$ denote the maps given by A.1.2 with $f(0)=p$ and $f^{\prime}(0)=p^{\prime}$. Suppose that for $x, x^{\prime} \in B_{\epsilon / 9}^{m}$ we have that $f(x)=f^{\prime}\left(x^{\prime}\right)$. Then there is a smooth embedding $h: B_{\epsilon / 9}^{m} \rightarrow B_{\epsilon}^{m}$ uniquely determined by Property (a) below; and there is an isometry $\bar{h}: \mathbf{R}^{m} \rightarrow \mathbf{R}^{m}$ of Euclidean space satisfying Properties (b) and (c) below:

(a) $h(x)=x^{\prime}$, and $f \mid B_{\epsilon / 9}^{m}=f^{\prime} \circ h$.

(b) $h(0)=\bar{h}(0)$, and $\left\|D(h-\bar{h})_{\mid z}\right\|<\mathcal{O}\left(\epsilon^{2}\right)$ at all $z \in B_{\epsilon / 9}^{m}$. [Here, and in Part (c) below, \|\| indicates the Euclidean norm of the appropriate derivative.] 
(c) There is a collection of positive numbers $\bar{B}=\left\{\bar{B}_{i}: i=1,2, \ldots\right\}$ which depend only on the $B=\left\{B_{i}\right\}$ and $\operatorname{dim} M$. We have for all $i$ and all $z \in B_{\epsilon / 9}^{m}$ that

$$
\left\|D^{i} h_{\mid z}\right\| \leq \bar{B}_{i}
$$

Remark A.1.3.1. Note that 1.3 (in the Proof of Theorem 0.5) can be deduced from A.1.3 in the special case when $p=p^{\prime}$ and $f=f^{\prime}$ : Just identify $x, y, \epsilon$ of 1.3 with $x, x^{\prime}, \epsilon$ of Corollary A.1.3; and assume (with no loss of generality) that $100 \delta<\epsilon$, where $\delta$ comes from 0.5 .

In the next theorem we let $p \in M$ and $f: B_{\epsilon}^{m} \rightarrow M$ be as in Theorem A.1.1 and Corollary A.1.2. We let $r: U \rightarrow B$ denote an infranil core for $M$; recall that $B$ is a ball in some Euclidean space $\mathbf{R}^{k}$. We set

$$
\begin{aligned}
\hat{U} & =f^{-1}(U), \\
\hat{r} & =r \circ f .
\end{aligned}
$$

So $\hat{r}$ maps $\hat{U}$ to $\hat{B}=B$. Let $K(r ; M)$ denote the curvature for $r$ in $(M, g)$ as defined in 0.1 ; and let $K\left(\hat{r} ; B_{\epsilon}^{m}\right)$ denote the curvatures for $\hat{r}$ in $\left(B_{\epsilon}^{m}, e\right)$ as defined in 0.1, where $e$ denotes the Euclidean metric.

Theorem A.1.4. Suppose that $U \subset f\left(B_{\epsilon}^{m}\right), K(r ; M) \leq \epsilon^{-1}$, and that $\left\|D r_{q}\right\|<\mathcal{O}(1)$ for all $q \in U$. Then we have that

$$
K\left(\hat{r} ; B_{\epsilon}^{m}\right)-\mathcal{O}(\epsilon)<K(r ; M)<K\left(\hat{r} ; B_{\epsilon}^{m}\right)+\mathcal{O}(\epsilon) .
$$

Remark A.1.5. The preceding theorem remains true if we replace $\hat{r}$ by $\hat{r} \mid X$, for any open subset $X \subset \hat{U}$ which satisfies $U \subset f(X)$. This theorem will be proven below.

In our next and last theorem of this appendix we let $r: U \rightarrow B$ denote an infranil core for $(M, g)$ of radius $\delta>0$, and we let $s: E \rightarrow B$ denote the thickening for $r$ described in 0.4. For each positive number $t>0$ we denote by $K(s ; M, t)$ the glb for all numbers $\sigma>0$ which satisfy

$$
\left.\| D s_{f(1)}-P_{s \circ f} \circ D s_{f(0)} \circ P_{f}^{-1}\right) \|<\sigma t
$$

for any smooth path $f:[0,1] \rightarrow E$ having length less than or equal to $t$. Here $P_{f}$ denotes parallel translation along $f$ in $(M, g)$, and $P_{s \circ f}$ denotes Euclidean-parallel translation along $s \circ f$ in $B$ (recall that $B$ is an open ball in some Euclidean space). Note (cf. 0.1 as applied to $s$ ) that

$$
K(s ; M)=\operatorname{lub}\{K(s ; M, t): 0<t\} .
$$


Theorem A.1.6. Let $\epsilon, \delta>0$ denote sufficiently small numbers, where how small is sufficient for $\epsilon$ depends only on $A=\left\{A_{i}\right\}$ and $\operatorname{dim} M$, and how small is sufficient for $\delta$ depends only on $\epsilon, A=\left\{A_{i}\right\}$, and $\operatorname{dim} M$. If $r$ satisfies Properties 0.2.2(a)-(c) for our present choice of $\epsilon, \delta>0$, then $s$ must satisfy

$$
K(s ; M, \delta)<\mathcal{O}(\epsilon) \delta^{-1}
$$

The following lemma will be used in the proof for Theorem A.1.6. In this lemma we let $\hat{s}: \hat{E} \rightarrow \hat{B}$ denote the composition function $\hat{E} \stackrel{f_{p}}{\longrightarrow} E \stackrel{s}{\longrightarrow} B$, where $f_{p}$ now denotes the immersion $f: B_{\epsilon}^{m} \rightarrow M$ of A.1.1 and A.1.2, and $\hat{E}=f_{p}^{-1}(E)$; and we let $X \subset \hat{E}$ denote an open subset of $\hat{E}$. For any number $t>0$ we denote by $K\left(\hat{s} \mid X ; B_{\epsilon}^{m}, t\right)$ the glb of all numbers $\sigma>0$ which satisfy

$$
\left\|D \hat{s}_{f(1)}-P_{\hat{s} \circ f} \circ D \hat{s}_{f(0)} \circ P_{f}^{-1}\right\|<\sigma t
$$

for any smooth path $f:[0,1] \rightarrow X$ which has Euclidean length less than or equal to $t$.

Lemma A.1.7. Suppose $E \subset f_{p}\left(B_{\epsilon}^{m}\right)$ and that for any smooth path $f$ : $[0,1] \rightarrow E$, having length less than or equal to $t$, there is another smooth path $\hat{f}:[0,1] \rightarrow X$ such that $f_{p} \circ \hat{f}=f$. Suppose also that $\left\|D s_{q}\right\|<\mathcal{O}(1)$ for all $q \in E$. Then we have that

$$
K\left(\hat{s} \mid X ; B_{\epsilon}^{m}, t\right)-\mathcal{O}(\epsilon)<K(s ; M, t)<3 K\left(\hat{s} \mid X ; B_{\epsilon}^{m}, t\right)+\mathcal{O}(\epsilon)
$$

provided $\epsilon$ is sufficiently small depending only on $A=\left\{A_{i}\right\}$ and $\operatorname{dim} M$.

The proof of Lemma A.1.7, which is analogous to the proof of Theorem A.1.4, is left as an exercise for the reader.

Proof of Corollary A.1.2. Recall Theorem A.1.1 which defines $\eta,\left\{B_{i}\right\}$ depending on $\left\{A_{i}\right\}$ and $\operatorname{dim} M$, such that for each $\epsilon \in(0, \eta)$ we have an immersion $f: B_{\epsilon}^{m} \rightarrow M$ and a pull back metric $\left\{g_{i, j}\right\}$ on $B_{\epsilon}^{m}$ satisfying Properties A.1.1(a) and (b). Let $\lambda_{m}$ be defined as below (A.1.2.1(c)); and choose $\epsilon$ as below (A.1.2.1(a)). Then Theorem A.1.1 implies that there is an immersion $f: B_{\epsilon}^{m} \rightarrow M$ such that the pulled back metric $\left\{g_{i, j}\right\}$ satisfies A.1.2.1(b). (Note that A.1.2.1(b) is true for any choice of $\lambda_{m}>0$ just so long as $\epsilon, B_{1}, \eta, \lambda_{m}$ are related as in A.1.2.1(a); the restriction placed on $\lambda_{m}$ in A.1.2.1(c) will be relevant later in this section.)

A.1.2.1. (a) $\epsilon<\min \left\{\lambda_{m} / B_{1}, \eta\right\}$.

(b) $\left|g_{i, j}(x)-\delta_{i, j}\right|<\lambda_{m}$, for all $x \in B_{\epsilon}^{m}$.

(c) $\lambda_{m}=\min \left\{(10 m)^{-m},\left(10^{-m}+1\right)^{1 / m}-1\right\}$. 
Let $\Gamma_{i, j}^{k}: B_{\epsilon}^{m} \rightarrow \mathbf{R}$ denote the classical Christoffel functions for the Riemannian metric represented by the $\left\{g_{i, j}\right\}$ with respect to the standard coordinates $x_{1}, x_{2}, \ldots, x_{m}$. Using of the equality

$$
\Gamma_{i, j}^{k}=1 / 2 \sum_{r=1}^{m} h_{k, r}\left(\partial g_{r, j} / \partial x_{i}+\partial g_{r, i} / \partial x_{j}+\partial g_{i, j} / \partial x_{r}\right)
$$

where the matrix $\left[h_{i, j}\right]$ is the inverse of the matrix $\left[g_{i, j}\right]$, together with A.1.1(b) and A.1.2.1(b) and (c), we can deduce the following:

A.1.2.2. $\left|\left(\partial^{n} \Gamma_{i, j}^{k} / \partial x_{s_{1}} \partial x_{s_{2}} \ldots \partial x_{s_{n}}\right)(x)\right|<C_{n}$ for all $n, i, j, k,\left\{s_{1}, \ldots, s_{n}\right\}$, $x \in B_{\epsilon}^{m}$.

Here $C=\left\{C_{i}\right\}$ is a collection of positive numbers which depend only on $B=\left\{B_{i}\right\}$ and $\operatorname{dim} M$.

The standard coordinates $x_{1}, x_{2}, \ldots, x_{m}$ for $\mathbf{R}^{m}$ induce coordinates on $T \mathbf{R}^{m} y_{1}, y_{2}, \ldots, y_{m}, y_{m+1}, \ldots, y_{2 m}$ via the correspondence

$$
\sum_{i=1}^{m} y_{m+i} \partial / \partial x_{i}\left(y_{1}, \ldots, y_{m}\right) \longleftrightarrow\left(y_{1}, y_{2}, \ldots, y_{m}, y_{m+1}, \ldots, y_{2 m}\right) .
$$

We define a vector field $V: T B_{\epsilon}^{m} \rightarrow T\left(T B_{\epsilon}^{m}\right)$ on $T B_{\epsilon}^{m}$ by A.1.2.3(a); note that we may deduce from A.1.2.2 that $V$ satisfies Property A.1.2.3(b) and (c).

A.1.2.3. (a) $V=\sum_{i=1}^{2 m} V_{i} \partial / \partial y_{i}$, where

$$
V_{i}\left(y_{1}, \ldots, y_{2 m}\right)= \begin{cases}y_{m+i} & \text { if } i \leq m \\ -\sum_{1 \leq j, k \leq m} y_{m+j} y_{m+k} \Gamma_{j, k}^{i}\left(y_{1}, \ldots, y_{m}\right) & \text { if } i>m .\end{cases}
$$

(b) For all $k, i,\left\{s_{1}, \ldots, s_{k}\right\}, y \in B_{\epsilon}^{m} \times B_{1}^{m}$ we have that

$$
\left|\partial^{k} V_{i} / \partial y_{s_{1}} \ldots \partial y_{s_{k}}(y)\right|<3^{k} m^{2}\left(\sum_{i=0}^{k} C_{i}\right)+1 .
$$

(c) For all $i$ and all $y \in B_{\epsilon}^{m} \times B_{1}^{m}$ we have that

$$
\left|V_{i}(y)\right| \leq \begin{cases}\left|y_{m+i}\right| & \text { if } i \leq m \\ m^{2} C_{0}\left(y_{m+1}^{2}+\cdots+y_{2 m}^{2}\right) & \text { if } i>m\end{cases}
$$

Note that in Parts (b) and(c) above we have used the identification $T \mathbf{R}^{m}=$ $\mathbf{R}^{m} \times \mathbf{R}^{m}$ - arising from the coordinates $\left(y_{1}, \ldots, y_{m}, y_{m+1}, \ldots, y_{2 m}\right)$ on $T \mathbf{R}^{m}$ — to make sense of " $y \in B_{\epsilon}^{m} \times B_{1}^{m}$ ". 
We integrate the vector field $V$ to get a partial flow $\psi: S \rightarrow T B_{\epsilon}^{m}$, where $S \subset \mathbf{R} \times T B_{\epsilon}^{m}$ is the maximal subset on which this partial flow makes sense. Note it follows from A.1.2.1(b) and (c) that the next two properties hold for $\psi$ :

A.1.2.4. (a) $[-2,2] \times\left(B_{\epsilon / 4}^{m} \times B_{\epsilon / 4}^{m}\right) \subset S$.

(b) $\psi\left([-2,2] \times\left(B_{\epsilon / 4}^{m} \times B_{\epsilon / 4}^{m}\right)\right) \subset B_{\epsilon}^{m} \times B_{\epsilon / 3}^{m}$.

[To see this notice that $\psi$ is the geodesic flow on $T B_{\epsilon}^{m}$ relative to the Riemannian metric $g_{i, j}$ on $B_{\epsilon}^{m}$.]

Now we can deduce from A.1.2.3(b) and A.1.2.4(b) that the partial flow

$$
\psi:[-2,2] \times\left(B_{\epsilon / 4}^{m} \times B_{\epsilon / 4}^{m}\right) \rightarrow T B_{\epsilon}^{m}
$$

satisfies:

A.1.2.5. $\left|\partial^{k} \psi / \partial y_{s_{1}} \ldots \partial y_{s_{k}}(t, y)\right| \leq D_{k}$

for all $k,(t, y) \in[-2,2] \times\left(B_{\epsilon / 4}^{m} \times B_{\epsilon / 4}^{m}\right)$, where | | denotes the Euclidean norm (with respect to the coordinates $y_{1}, y_{2}, \ldots, y_{2 m}$ ). Here $D=\left\{D_{i}\right\}$ is collection of positive numbers which depend only on $C=\left\{C_{i}\right\}$ and $m=\operatorname{dim} M$.

To complete the proof for Corollary A.1.2 recall that $\psi: S \rightarrow T B_{\epsilon}^{m}$ is the (partial) geodesic flow for the Riemannian metric $g_{i, j} d x_{i} d x_{j}$ on $B_{\epsilon}^{m}$. Thus the composition

$$
B_{\epsilon / 4}^{m}=0 \times B_{\epsilon / 4}^{m} \subset B_{\epsilon / 4}^{m} \times B_{\epsilon / 4}^{m} \stackrel{\psi_{1}}{\longrightarrow} T B_{\epsilon}^{m} \stackrel{\text { proj. }}{\longrightarrow} B_{\epsilon}^{m} \stackrel{f}{\longrightarrow} M
$$

is equal to a composition

$$
B_{\epsilon / 4}^{m} \stackrel{L}{\longrightarrow} T M_{p} \stackrel{\exp }{\longrightarrow} M,
$$

where $L$ is a linear isometry and exp is the exponential map and $f$ is given in A.1.1 and $\psi_{1}$ is the composition

$$
B_{\epsilon / 4}^{m} \times B_{\epsilon / 4}^{m}=1 \times B_{\epsilon / 4}^{m} \times B_{\epsilon / 4}^{m} \subset S \stackrel{\psi}{\longrightarrow} T B_{\epsilon}^{m} .
$$

Thus it follows from A.1.1(b) and from A.1.2.5 that $\exp \circ L$ satisfies:

A.1.2.6. $\left|\partial^{k} \bar{g}_{i, j} / \partial x_{s_{1}} \ldots \partial x_{s_{k}}(x)\right| \leq \bar{B}_{k}$

for all $k$ and all $x \in B_{\epsilon / 4}^{m}$, where the $\bar{B}=\left\{\bar{B}_{i}\right\}$ is a set of positive numbers which depend only on the $B=\left\{B_{i}\right\}$ and $m=\operatorname{dim} M$, and the $\bar{g}_{i, j}: T B_{\bar{\epsilon}}^{m} \rightarrow$ $\mathbf{R}$ are defined by

$$
\bar{g}_{i, j}(x)=g\left(D(\exp \circ L)\left(\partial / \partial x_{i}(x)\right), D(\exp \circ L)\left(\partial / \partial x_{j}(x)\right)\right) .
$$

[Recall that $B_{\epsilon / 4}^{m}=0 \times B_{\epsilon / 4}^{m} \subset B_{\epsilon}^{m} \times \mathbf{R}^{m}$, and $B_{\epsilon}^{m} \times \mathbf{R}^{m}$ has coordinates $y_{1}, \ldots, y_{2 m}$. Here we have renamed the coordinates $y_{m+1}, y_{m+2}, \ldots, y_{2 m}$ 
for $\mathbf{R}^{m}$ to be $x_{1}, x_{2}, \ldots, x_{m}$.] If we replace (in A.1.1) the $f, B, g_{i, j}, \epsilon$ by $\bar{f}=\exp \circ L, \bar{B}, \bar{g}_{i, j}, \bar{\epsilon}=\epsilon / 4$, then A.1.2.6 assures that Property A.1.1(b) is still satisfied for the "barred" quantities; Property A.1.1(a) is satisfied by the "barred" quantities because $\bar{f}$ (restricted to a small neighborhood for $\left.0 \in B_{\bar{\epsilon}}^{m}\right)$ is a normal coordinate system at $p$.

This completes the proof for Corollary A.1.2.

Proof of Corollary A.1.3. Note that all the steps in the proof for A.1.2 apply to the immersions $f, f^{\prime}: B_{\epsilon}^{m} \rightarrow M$ given in the hypothesis of A.1.3. In particular Properties A.1.2.1(b) and (c) hold for the functions $\left\{g_{i, j}\right\}$ and $\left\{g_{i, j}^{\prime}\right\}$ associated to $f$ and $f^{\prime}$ respectively by A.1.1. For any $y \in B_{\epsilon / 9}^{m}$ let $h_{y}:[0,1] \rightarrow B_{\epsilon}^{m}$ be defined by $h_{y}(t)=(1-t) x+t y, t \in[0,1]$; note that length $\left(h_{y}\right)=|y-x|<2 \epsilon / 9$, where the length and the norm are computed in Euclidean metric. Now it follows from A.1.2.1(b) and (c) as applied to both the $\left\{g_{i, j}\right\}$ and the $\left\{g_{i, j}^{\prime}\right\}$, and from the fact that both $f$ and $f^{\prime}$ are immersions, and from the fact that $x^{\prime} \in B_{\epsilon / 9}^{m}$, that there is a smooth path $h_{y}^{\prime}:[0,1] \rightarrow B_{\epsilon}^{m}$ which satisfies the following properties:

A.1.3.1. (a) $f \circ h_{y}(t)=f^{\prime} \circ h_{y}^{\prime}(t)$ for all $t \in[0,1]$.

(b) length $\left(h_{y}^{\prime}\right)<\operatorname{length}\left(h_{y}\right)+\epsilon^{2} m^{2}(10 m)^{-m}$, where the lengths are computed in the Euclidean metric.

Now we can define $h: B_{\epsilon / 9}^{m} \rightarrow B_{\epsilon}^{m}$ by:

A.1.3.1. (c) $h(y)=h_{y}^{\prime}(1)$

for all $y \in B_{\epsilon / 9}^{m}$. We leave as an exercise for the reader to determine that $h$ is a smooth embedding uniquely determined by the properties listed in A.1.3.1.

In order to verify Properties A.1.3(b) and (c) for $h: B_{\epsilon / 9}^{m} \rightarrow B_{\epsilon}^{m}$ we will need another description of this map. Let $\psi: S \rightarrow T B_{\epsilon}^{m}$ and $\psi^{\prime}: S^{\prime} \rightarrow T B_{\epsilon}^{m}$ denote the (partial) geodesic flows for $g_{i, j} d x_{i} d x_{j}$ and $g_{i, j}^{\prime} d x_{i} d x_{j}$ respectively. We have by A.1.2.4 that $[-2,2] \times\left(B_{\epsilon / 4}^{m} \times B_{\epsilon / 4}^{m}\right) \subset S$ and $[-2,2] \times\left(B_{\epsilon / 4}^{m} \times\right.$ $\left.B_{\epsilon / 4}^{m}\right) \subset S^{\prime}$. Define mappings $r: B_{\epsilon / 4}^{m} \rightarrow B_{\epsilon}^{m}$ and $r^{\prime}: B_{\epsilon / 4}^{m} \rightarrow B_{\epsilon}^{m}$ to be the compositions:

A.1.3.2. (a)

$$
\begin{aligned}
B_{\epsilon / 4}^{m}=(x) \times B_{\epsilon / 4}^{m} \subset S \stackrel{\psi_{1}}{\longrightarrow} T B_{\epsilon}^{m}=B_{\epsilon}^{m} \times \mathbf{R}^{m} \stackrel{\text { proj. }}{\longrightarrow} B_{\epsilon}^{m} \\
\downarrow
\end{aligned}
$$


(b)

$$
\begin{aligned}
B_{\epsilon / 4}^{m}=\left(x^{\prime}\right) \times B_{\epsilon / 4}^{m} \subset S \stackrel{\psi_{1}^{\prime}}{\longrightarrow} T B_{\epsilon}^{m}=B_{\epsilon}^{m} \times \mathbf{R}^{m} \stackrel{\text { proj. }}{\longrightarrow} B_{\epsilon}^{m} \\
\downarrow r^{\prime}
\end{aligned}
$$

respectively. Define the linear map $L: \mathbf{R}^{m} \rightarrow \mathbf{R}^{m}$ to be the composition:

A.1.3.2. (c)

$$
\mathbf{R}^{m}=T B_{\epsilon \mid x}^{m} \stackrel{D f}{\longrightarrow} T M_{\mid f(x)} \stackrel{\left(D f^{\prime}\right)^{-1}}{\longrightarrow} T B_{\epsilon \mid x^{\prime}}^{m}=\mathbf{R}^{m},
$$

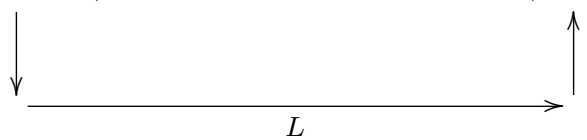

where the identifications $\mathbf{R}^{m}=T B_{\epsilon \mid x}^{m}$ and $T B_{\epsilon \mid x^{\prime}}^{m}=\mathbf{R}^{m}$ are induced from the standard coordinates $x_{1}, x_{2}, \ldots, x_{m}$ for $B_{\epsilon}^{m}$. Note that the diffeomorphism $h: B_{\epsilon / 9}^{m} \rightarrow B_{\epsilon}^{m}$ defined in A.1.3.1(c) is also given by the following formula:
A.1.3.2.
(d) $h=r^{\prime} \circ L \circ r^{-1} \mid B_{\epsilon / 9}^{m}$.

[Note that $r, r^{\prime}$ are essentially the exponential maps for $g_{i, j} d x_{i} d x_{j}$ and $g_{i, j}^{\prime} d x_{i} d x_{j}$ respectively. Thus Image $(r)$ is the ball $B_{\epsilon / 4, x}$ of radius $\epsilon / 4$ centered at $x$ in $B_{\epsilon / 4}^{m}$ computed with respect to the metric $g_{i, j} d x_{i} d x_{j}$. Note that A.1.2.1(b) and (c) (applied just to the $\left\{g_{i, j}\right\}$ ), together with the fact that $x \in B_{\epsilon / 9}^{m}$, assure us that:

(i) $B_{\epsilon / 9}^{m} \subset B_{\epsilon / 4, x}$.

Thus $r^{-1}: B_{\epsilon / 9}^{m} \rightarrow B_{\epsilon / 4}^{m}$ is well-defined. In addition A.1.2.1(b) and (c) (applied to both the $\left\{g_{i, j}\right\}$ and the $\left\{g_{i, j}^{\prime}\right\}$ ), assure us that:

(ii) $L \circ r^{-1}\left(B_{\epsilon / 9}^{m}\right) \subset B_{\epsilon / 4}^{m}$.

Thus (by (i) and (ii)) we have that $r^{\prime} \circ L \circ r^{-1}$ is well defined on $B_{\epsilon / 9}^{m}$ (the domain of $h$ ); this is a necessary condition for A.1.3.2 to hold.]

Note that Properties A.1.3(b) and (c) follow easily from A.1.3.2(d) and the following two claims:

Claim A.1.3.3. For every $v \in \mathbf{R}^{m}$ we have that

$$
\left(1-\mathcal{O}\left(\epsilon^{2}\right)\right)|v| \leq|L(v)| \leq\left(1+\mathcal{O}\left(\epsilon^{2}\right)\right)|v|,
$$

where $\mid$ | denotes the Euclidean norm. 
Claim A.1.3.4. Let $\bar{r}, \bar{r}^{\prime}: \mathbf{R}^{m} \rightarrow \mathbf{R}^{m}$ denote the translations of Euclidean space which satisfy $\bar{r}(0)=x$ and $\bar{r}^{\prime}(0)=x^{\prime}$. There is a collection of positive numbers $E=\left\{E_{i}\right\}$ which depend only on $m=\operatorname{dim} M$ and on the $B=\left\{B_{i}\right\}$ of A.1.1, such that the following properties hold:

(a) $\left\|D(r-\bar{r})_{\mid z}\right\|<\mathcal{O}\left(\epsilon^{2}\right)$ and $\left\|D\left(r^{\prime}-\bar{r}^{\prime}\right)_{\mid z}\right\|<\mathcal{O}\left(\epsilon^{2}\right)$ hold for all $z \in B_{\epsilon / 4}^{m}$.

(b) $\left\|D^{i} r_{\mid z}\right\|<E_{i}$ and $\left\|D^{i} r_{\mid z}^{\prime}\right\|<E_{i}$ for all $i$ and all $z \in B_{\epsilon / 4}^{m}$.

\section{Verification of Claim A.1.3.3.}

The key idea here is to improve upon Properties A.1.2.1(b) and (c) for both the $\left\{g_{i, j}\right\}$ and the $\left\{g_{i, j}^{\prime}\right\}$ (cf. A.1.3.3.2 below). Since $f, f^{\prime}: B_{\epsilon}^{m} \rightarrow M$ are (when restricted to a sufficiently small neighborhood for $0 \in B_{\epsilon}^{m}$ ) normal coordinates for $M$ near $p, p^{\prime} \in M$ respectively, it follows that:

A.1.3.3.1. $\partial g_{i, j} / \partial x_{k}(0)=0=\partial g_{i, j}^{\prime} / \partial x_{k}(0)$ for all $i, j, k$.

Property A.1.3.3.1, together with Properties A.1.1(a) and (b) for both the $\left\{g_{i, j}\right\}$ and the $\left\{g_{i, j}^{\prime}\right\}$, imply that:

A.1.3.3.2. $\left|g_{i, j}(z)-\delta_{j}^{i}\right| \leq \mathcal{O}\left(\epsilon^{2}\right)$ and $\left|g_{i, j}^{\prime}(z)-\delta_{j}^{i}\right| \leq \mathcal{O}\left(\epsilon^{2}\right)$

for all $i, j$ and all $z \in B_{\epsilon}^{m}$. By reviewing the definition given for the $\left\{g_{i, j}\right\}$ and the $\left\{g_{i, j}^{\prime}\right\}$ just prior to A.1.1, we see it follows from A.1.3.2(c) that:

A.1.3.3.3. $\sum_{i, j} g_{i, j}(x) v_{i} v_{j}=\sum_{i, j} g_{i, j}^{\prime}\left(x^{\prime}\right) L(v)_{i} L(v)_{j}$

for all $v \in \mathbf{R}^{m}$, where $\left(v_{1}, v_{2}, \ldots, v_{m}\right)$ and $\left(L(v)_{1}, L(v)_{2}, \ldots, L(v)_{m}\right)$ denote the standard coordinates for $v$ and $L(v)$. Now Claim A.1.3.3 can be easily deduced from A.1.3.3.2 and A.1.3.3.3.

\section{Verification of Claim A.1.3.4.}

Note that A.1.3.4(b) can easily be deduced from A.1.2.5 (as applied to both $\psi, \psi^{\prime}$ ), and from A.1.3.2(a) and (b).

Now we will verify A.1.3.4(a); the key idea here is to improve upon Property A.1.2.5 in the special case that $k=2$ in A.1.2.5 (cf. A.1.3.4.4 below). Towards this end we first note it follows from A.1.3.3.1 that:

$$
\text { A.1.3.4.1. } \Gamma_{j, k}^{i}(0)=0=\Gamma_{j, k}^{i}(0)
$$

for all $i, j, k$, where $\Gamma_{j, k}^{i}$ and $\Gamma_{j, k}^{i}$ are the Christoffel functions associated to the $g_{i, j} d x_{i} d x_{j}$ and $g_{i, j}^{\prime} d x_{i} d x_{j}$. We deduce from A.1.2.2 (as applied to both $\Gamma_{j, k}^{i}$ and $\Gamma_{j, k}^{i}$ ) and from A.1.3.4.1, that:

A.1.3.4.2. $\left|\Gamma_{j, k}^{i}(z)\right| \leq \mathcal{O}(\epsilon)$ and $\left|\Gamma_{j, k}^{i}(z)\right| \leq \mathcal{O}(\epsilon)$ 
for all $i, j, k$ and all $z \in B_{\epsilon}^{m}$. Now let $V$ and $V^{\prime}$ denote the vector fields on $T B_{\epsilon}^{m}$ defined in A.1.2.3(a) (where $\Gamma_{j, k}^{i}$ replaces $\Gamma_{j, k}^{i}$ in A.1.2.3(a) to define $\left.V^{\prime}\right)$. Then it follows from A.1.2.3(a) and from A.1.3.4.2 that:

$$
\text { A.1.3.4.3. }\left|\partial^{2} V / \partial y_{i_{1}} \partial y_{i_{2}}(y)\right| \leq \mathcal{O}(\epsilon) \text { and }\left|\partial^{2} V^{\prime} / \partial y_{i_{1}} \partial y_{i_{2}}(y)\right| \leq \mathcal{O}(\epsilon)
$$

for all $\left(i_{1}, i_{2}\right)$ and all $y \in B_{\epsilon}^{m} \times B_{\epsilon}^{m}$, where $\left(y_{1}, \ldots, y_{2 m}\right)$ are the standard coordinates for $T B_{\epsilon}^{m}$ referred to in A.1.2.3 and where || indicates the Euclidean norm for vectors. Since we integrate $V$ and $V^{\prime}$ to get the (partial) geodesic flows $\psi$, and $\psi^{\prime}$, we may deduce the following inequalities from those in A.1.3.4.3:

A.1.3.4.4. $\left|\partial^{2} \psi / \partial y_{i_{1}} \partial y_{i_{2}}(t, y)\right| \leq \mathcal{O}(\epsilon)$ and $\left|\partial^{2} \psi^{\prime} / \partial y_{i_{1}} \partial y_{i_{2}}(t, y)\right| \leq \mathcal{O}(\epsilon)$ for all $\left(i_{1}, i_{2}\right)$ and all $(t, y) \in[-2,2] \times\left(B_{\epsilon / 4}^{m} \times B_{\epsilon / 4}^{m}\right)$.

To complete the verification for A.1.3.4(a) note that the following equalities are a consequence of the definition given for $r, r^{\prime}$ in A.1.3.2(a) and (b):

A.1.3.4.5. $D(r-\bar{r})_{\mid 0}=0=D\left(r^{\prime}-\bar{r}^{\prime}\right)_{\mid 0}$.

Now Claim A.1.3.4(a) is easily deduced from A.1.3.2(a) and (b), A.1.3.4.4, and A.1.3.4.5.

This completes the proof for Corollary A.1.3.

Proof of Theorem A.1.4. For each smooth path $p:[0,1] \rightarrow B_{\epsilon}^{m}$ let $P_{p, g}$ and $P_{p, e}$ denote parallel translation along $p$ with respect to the metrics $g=g_{i, j} d x_{i} d x_{j}$ and $e=\delta_{j}^{i} d x_{i} d x_{j}$ respectively. The following relation between $P_{p, g}$ and $P_{p, e}$ is an easy consequence of Property A.1.3.4.2 (as applied to the Christoffel functions $\Gamma_{i, j}^{k}$ of $\left.g_{i, j} d x_{i} d x_{j}\right)$.

A.1.4.1. For each smooth path $p:[0,1] \rightarrow B_{\epsilon}^{m}$ and each vector $v \in T B_{\epsilon \mid p(0)}^{m}$ we have

$$
\left|P_{p, g}(v)-P_{p, e}(v)\right|_{e}<\left(\operatorname{length}_{e}(p)\right) \mathcal{O}(\epsilon)
$$

Now Theorem A.1.4 is a direct consequence of Property A.1.4.1, and of Property A.1.3.3.2 (as applied to the $g_{i, j}$ ), and of the hypothesis for A.1.4 that $\left\|D r_{q}\right\|<\mathcal{O}(1)$ for all $q \in \widetilde{U}$.

This completes the proof for Theorem A.1.4.

Proof of Theorem A.1.6. Note from the hypothesis of A.1.6 (that $r$ satisfies $0.2 .2(\mathrm{a})-(\mathrm{c}))$ that:

A.1.6.1. (a) diameter $(U)<\mathcal{O}(\delta)$.

Thus, by choosing $p \in U$ and choosing $\delta \ll \epsilon$, we may assume that: 
A.1.6.1. (b) $U \subset f_{p}\left(B_{\epsilon / 3}^{m}\right)$.

In particular A.1.4 applies to $r$ and $\hat{r}$, where $\hat{r}: \hat{U} \rightarrow \hat{B}$ was defined just prior to A.1.4. We also want to apply A.1.7 to $s$ and $\hat{s}$, where $\hat{s}: \hat{E} \rightarrow \hat{B}$ was defined just prior to A.1.7; recall that $\hat{E}=f_{p}^{-1}(E)$.

The remainder of this proof is based upon the following claim, which we will verify at the end of this proof. In this claim we set $k=\operatorname{dim} \hat{U}$, and for any integer $i>0$ and any number $t>0$ we let $B_{t}^{i}$ denote the open ball of radius $t$ centered at the origin of $\mathbf{R}^{i}$.

Claim A.1.6.2. Let $\hat{Y}=\hat{U} \cap B_{5 \delta}^{m}$. There is a smooth embedding $h$ : $Y \times B_{\delta}^{m-k} \rightarrow B_{\epsilon}^{m}$, where $Y$ is an open subset of $B_{6 \delta}^{k}$ containing 0 , which satisfies the following properties:

(a) $h(0,0)=0$, and $D h_{(0,0)}$ is an isometry with respect to the Euclidean metrics.

(b) $\left\|D h_{(x, y)}-D h_{\left(x^{\prime}, y^{\prime}\right)}\right\|<\mathcal{O}(\epsilon)$ for all $(x, y),\left(x^{\prime}, y^{\prime}\right) \in Y \times B_{\delta}^{m-k}$. (Here the norm \|\| is computed with respect to the Euclidean metrics.)

(c) $h(Y, 0)=\hat{Y}$ and $h\left(z \times B_{\delta}^{m-k}\right)=\hat{\rho}^{-1}(h(z, 0))$ for all $z \in Y$, where

$$
\hat{\rho}: \hat{E} \cap B_{6 \delta}^{m} \rightarrow \hat{U}
$$

denotes the orthogonal projection with respect to the Riemannian metric $g_{i, j}$ of A.1.1.

To complete the proof for Theorem A.1.6 we first note we have as an immediate consequence of A.1.6.2(a) and (b) the following properties. Let $\rho: Y \times B_{\delta}^{m-k} \rightarrow Y$ denote the standard projection onto the first factor.

A.1.6.3. (a) $\left\|D\left(\rho \circ h^{-1}\right)_{q}\right\|<1+\mathcal{O}(\epsilon)$ for all $q \in h\left(Y \times B_{\delta}^{m-k}\right)$.

(b) $\left\|D\left(\rho \circ h^{-1}\right)_{q}-D\left(\rho \circ h^{-1}\right)_{q^{\prime}}\right\|<\mathcal{O}(\epsilon)$ for all $q, q^{\prime} \in h\left(Y \times B_{\delta}^{m-k}\right)$.

Note also that it follows from A.1.6.2(a) and (b) as applied to $h \mid Y \times 0$ (see also A.1.6.2(c)), and from 0.2.2(a)-(c) as applied to $r$, and from A.1.1 and A.1.4 as applied to $r$ and $\hat{r}$, that:

A.1.6.3. (c) $\left\|D(\hat{r} \circ h \circ I)_{z}\right\|<1+\mathcal{O}(\epsilon)$ for all $z \in Y$,

(d) $\left\|D(\hat{r} \circ h \circ I)_{z}-D(\hat{r} \circ h \circ I)_{z^{\prime}}\right\|<\mathcal{O}(\epsilon)$ for all $z, z^{\prime} \in Y$,

where $I: Y \rightarrow Y \times 0$ sends $z$ to $(z, 0)$. Finally we note that A.1.6.2(c) implies that:

A.1.6.3. (e) $(\hat{r} \circ h \circ I) \circ\left(\rho \circ h^{-1}\right)=\hat{s} \mid h\left(Y \times B_{\delta}^{m-k}\right)$.

It follows from A.1.6.3(a)-(e) that:

A.1.6.4. (a) $\left\|D \hat{s}_{q}\right\|<1+\mathcal{O}(\epsilon)$ for all $q \in h\left(Y \times B_{\delta}^{m-k}\right)$ 
(b) $\left\|D \hat{s}_{q}-D \hat{s}_{q^{\prime}}\right\|<\mathcal{O}(\epsilon)$ for all $q, q^{\prime} \in h\left(Y \times B_{\delta}^{m-k}\right)$.

We may rephrase A.1.6.4(b) as follows. (See Remark A.1.7 for notation.)

A.1.6.4. (c) $K\left(\hat{s} \mid X ; B_{\epsilon}^{m}, \delta\right)<\mathcal{O}(\epsilon) \delta^{-1}$ where $X=h\left(Y \times B_{\delta}^{m-k}\right)$.

Note that it follows from A.1.6.2(a), (b) and (c), and from A.1.3.3.2 as applied to $g_{i, j}$, and from $0.2 .2(\mathrm{a})$-(c) as applied to $r$, that the subset $X \subset \hat{E}$ of A.1.6.4(c) has the following desirable lifting property:

A.1.6.4. (d) For each smooth path $f:[0,1] \rightarrow E$ having length less than or equal to $\delta$, there is a smooth path $\hat{f}:[0,1] \rightarrow X$ such that $f_{p} \circ \hat{f}=f$.

Now the conclusion of A.1.6 follows immediately from Property A.1.6.4(c) and Lemma A.1.7: Note that Properties A.1.6.4(a) and (d), and Property A.1.3.3.2 as applied to $g_{i, j}$ assure that the hypotheses of Lemma A.1.7 hold.

\section{Verification of Claim A.1.6.2.}

Let $V$ denote the tangent plane to $\hat{U}$ at $0 \in \hat{U}$, and choose an isometry $g_{1}: \mathbf{R}^{k} \rightarrow V$ with $g_{1}(0)=0$ (here $V$ has the metric inherited from the Euclidean metric on $B_{\epsilon}^{m}$ ). For sufficiently small $\delta$ there is a unique smooth embedding $g_{2}: Y \rightarrow B_{\epsilon}^{m}$ which satisfies the following properties:

\section{A.1.6.2.1.}

(a) We have that $g_{2}(Y)=\hat{Y}$ and, for all $z \in Y, g_{2}(z)-g_{1}(z)$ is perpendicular to $V$. (To make sense of the difference $g_{2}(z)-g_{1}(z)$ we identify $g_{1}(z)$ with its image in $B_{\epsilon}^{m}$ under the Euclidean-exponential map.)

(b) $g_{2}(0)=0$; and $D g_{2} \mid T\left(B_{6 \delta}^{k}\right)_{0}=g_{1}$ when $T\left(B_{\delta}^{k}\right)_{0}$ is identified with $\mathbf{R}^{k}$ by the Euclidean exponential map.

(c) $\left\|D^{2}\left(g_{2}\right)_{q}\right\|<\mathcal{O}(\epsilon) \delta^{-1}$ for all $q \in Y$.

We note that $Y$ and $g_{2}$ are uniquely determined from $g_{1}$ by Property A.1.6.2.1(a); and Property A.1.6.2.1(b) is a direct consequence of A.1.6.2.1(a) and of the definition for $g_{1}$. Note that Property A.1.6.2.1(c) is equivalent to the inequality:

(i) $K\left(\operatorname{Image}\left(g_{2}\right) ; B_{\epsilon}^{m}\right)<\mathcal{O}(\epsilon) \delta^{-1}$;

and, since Image $\left(g_{2}\right)$ is an open subset of $\hat{U}$, this last inequality is implied by:

(ii) $K\left(\hat{U} ; B_{\epsilon}^{m}\right)<\mathcal{O}(\epsilon) \delta^{-1}$.

(Here for any smooth submanifold $S \subset B_{\epsilon}^{m}$ we denote by $K\left(S ; B_{\epsilon}^{m}\right.$ ) the curvature of the constant map $c: S \rightarrow\{1\}$ with respect to the Euclidean metric on $B_{\epsilon}^{m}$ as described in 0.1.) Finally we note that inequality (ii) is a consequence of 0.2.2(a) and A.1.4. (Note that Properties 0.2.2(a)-(c) are 
assumed in A.1.6; and A.1.4 may be applied here because its hypotheses are a consequence of 0.2.2(a)-(c) and A.1.3.3.2.)

Now we define smooth vector fields $v_{1}, v_{2}, \ldots, v_{m-k}$ on $\hat{Y}=g_{2}(Y)$ as follows: Let $T \hat{U}^{\perp}$ denote the orthogonal complement for $T \hat{U}$ in $T\left(B_{\epsilon}^{m}\right)_{\mid \hat{U}}$ with respect to the metric $\left\{g_{i, j}\right\}$; and for each $z \in Y$ let $\rho_{z}: T\left(B_{\epsilon}^{m}\right)_{g_{2}(z)} \rightarrow T \hat{U}_{g_{2}(z)}^{\perp}$ denote the orthogonal projection with respect to the metric $\left\{g_{i, j}\right\}$. Choose the $\left\{v_{i}(0)\right\}$ to be an orthonormal basis for $T \hat{U}_{0}^{\perp}$ with respect to the metric $\left\{g_{i, j}\right\}$; and then define $\left\{v_{1}(z), v_{2}(z), \ldots, v_{m-k}(z)\right\}$ to be the orthonormal subset of $T \hat{U}_{g_{2}(z)}^{\perp}$ obtained by applying the Gramm-Schmidt process to the set of vectors $\left\{\rho_{z} \circ P_{z}\left(v_{1}(0)\right), \rho_{z} \circ P_{z}\left(v_{2}(0)\right), \ldots, \rho_{z} \circ P_{z}\left(v_{m-k}(0)\right)\right\}$ with respect to the metric $\left\{g_{i, j}\right\}$, where $P_{z}$ denotes Euclidean parallel translation to $T\left(B_{\epsilon}^{m}\right)_{g_{2}(z)}$. Note that $T\left(B_{\epsilon}^{m}\right)$ is identified with $B_{\epsilon}^{m} \times \mathbf{R}^{m}$ by using Euclidean parallel translation to identify each $T\left(B_{\epsilon}^{m}\right)_{y}, y \in B_{\epsilon}^{m}$, with $T\left(B_{\epsilon}^{m}\right)_{0}$, and by identifying $T\left(B_{\epsilon}^{m}\right)_{0}$ with $\mathbf{R}^{m}$ via the exponential map exp $: T\left(B_{\epsilon}^{m}\right) \rightarrow \mathbf{R}^{m}$. Thus we may define maps $w_{i}: Y \rightarrow \mathbf{R}^{m}$ (for $1 \leq i \leq m-k)$ by $\left(g_{2}(z), w_{i}(z)\right)=v_{i}\left(g_{2}(z)\right)$ for all $z \in Y$. Note that it follows from the construction just given, and from Properties A.1.6.2.1(a)(c), and from Properties A.1.1(a) and (b), that the maps $\left\{w_{i}\right\}$ satisfy the following properties:

A.1.6.2.2. (a) For each $z \in Y$ the vectors $\left\{\left(g_{2}(z), w_{i}(z)\right): 1 \leq i \leq m-k\right\}$ are an orthonormal basis for $T \hat{U}^{\perp}$ with respect to the metric $\left\{g_{i, j}\right\}$.

(b) $\left\|D\left(w_{i}\right)_{z}\right\|<\mathcal{O}(\epsilon) \delta^{-1}$ (where the norm \|\| is computed with respect to the Euclidean metrics on $B_{6 \delta}^{k}$ and on $\left.T\left(B_{\epsilon}^{m}\right)\right)$.

We can now define a map $g_{3}: Y \times B_{\delta}^{m-k} \rightarrow T\left(B_{\epsilon}^{m}\right)=B_{\epsilon}^{m} \times \mathbf{R}^{m}$ by:

A.1.6.2.3. $g_{3}(z, a)=\left(g_{2}(z), \sum_{i=1}^{m-k} a_{i} w_{i}(z)\right)$

for all $(z, a) \in Y \times B_{\delta}^{m-k}$, where $\left(a_{1}, \ldots, a_{m-k}\right)$ are the standard coordinates for $a \in B_{\delta}^{m-k}$. It follows from A.1.6.2.1-A.1.6.2.3, and from A.1.1(a) and (b), that $g_{3}$ is a smooth embedding which satisfies the following properties. (See in particular A.1.6.2.1(b) and A.1.1(a) for Part (a) below.)

A.1.6.2.4. (a) $g_{3}(0,0)=(0,0)$; and $D\left(g_{3}\right)_{(0,0)}$ is a linear isometry with respect to the Euclidean metrics.

(b) $\left\|D\left(g_{3}\right)_{(z, a)}-D\left(g_{3}\right)_{\left(z^{\prime}, a^{\prime}\right)}\right\|<\mathcal{O}(\epsilon)$ for all $(z, a)$ and $\left(z^{\prime}, a^{\prime}\right)$ in $Y \times B_{\delta}^{m-k}$. (Here the norm \|\| is computed with respect to the Euclidean metrics on $B_{6 \delta}^{k} \times B_{\delta}^{m-k}$ and $T\left(B_{\epsilon}^{m}\right)=B_{\epsilon}^{m} \times \mathbf{R}^{m}$.) 
(c) $g_{3}(z, 0)=\left(g_{2}(z), 0\right)$, so $g_{3}(z, 0) \in \hat{U}$ for all $z \in Y$; moreover $g_{3}(z \times$ $\left.B_{\delta}^{m-k}\right)=\pi^{-1}\left(g_{3}(z, 0)\right)$ for all $z \in Y$, where $\pi:\left(T \hat{U}^{\perp}\right)^{\delta} \rightarrow \hat{U}$ denotes the usual bundle projection and $\left(T \hat{U}^{\perp}\right)^{\delta}$ denotes all vectors $v \in T \hat{U}^{\perp}$ whose $\left\{g_{i j}\right\}$-length is less than $\delta$.

Now we can finally define the map $h: Y \times B_{\delta}^{m-k} \rightarrow B_{\epsilon}^{m}$ of A.1.6.2 to be the composition:

A.1.6.2.5. $Y \times B_{\delta}^{m-k} \stackrel{g_{3}}{\longrightarrow} T\left(B_{\epsilon}^{m}\right)=B_{\epsilon}^{m} \times \mathbf{R}^{m} \supset B_{\epsilon / 4}^{m} \times B_{\epsilon / 4}^{m} \stackrel{\exp }{\longrightarrow} B_{\epsilon}^{m}$,

where "exp" denotes the exponential map with respect to the metric $\left\{g_{i, j}\right\}$ on $B_{\epsilon}^{m}$. It follows from A.1.1(a) and (b) that "exp" is defined on $B_{\epsilon / 4}^{m} \times B_{\epsilon / 4}^{m}$ (see also A.1.2.4, and note that $\exp =\psi_{1}$ ). And, by choosing $\delta \ll \epsilon$, it follows from the definition of $g_{3}$ in A.1.6.2.3 that Image $\left(g_{3}\right) \subset B_{\epsilon / 4}^{m} \times B_{\epsilon / 4}^{m}$ (see also A.1.6.2.1). Thus $h$ is well-defined by the preceding composition. Note that Property A.1.6.2(a) is implied by A.1.6.2.4(a) and A.1.6.2.3 and A.1.6.2.5. Note also that Property A.1.6.2(c) is implied by A.1.6.2.4(c) and A.1.6.2.5. Finally we note that Property A.1.6.2(b) is implied by A.1.6.2.4(a) and (b) and A.1.6.2.5.

This completes the verification of Claim A.1.6.2; thus also the proof of Theorem A.1.6 is completed.

\section{Appendix 2.}

In this appendix we review the results for the Collapsing theory of CheegerFukaya-Gromov (cf. [4]) which we need to carry out the proof of Theorem 0.3 in $\S 3$ above (cf. A.2.2-A.2.5 below).

Let $M, g_{M}$ denote an $A$-regular complete Riemannian manifold, where $A$ denotes a collection $\left\{A_{i}: i=0,1,2, \ldots\right\}$ of positive numbers. For any $\delta>0$ we shall say that $g_{M}$ is $\delta$-round at the point $p \in M$ if there is an open neighborhood $V \subset M$ for $p \in M$, and a regular covering $\pi: \hat{V} \rightarrow V$ for $V$ with covering group $\Lambda$, such that the following properties hold. Note that $\hat{V}$ is equipped with a Riemannian metric $g_{\hat{V}}$ gotten by pulling back $g_{M}$ along $\pi: \hat{V} \rightarrow V$.

A.2.1. (a) $V$ contains the ball $B(p, \delta)$ of radius $\delta$ centered at $p \in M$.

(b) The injectivity radius of $\hat{V}$ at all points of $\pi^{-1}(B(p, \delta))$ is greater than $\delta$.

(c) There is a virtually nilpotent Lie group $H$ and an effective isometric action $H \times \hat{V} \rightarrow \hat{V}$ extending that of $\Lambda \times \hat{V} \rightarrow \hat{V}$. (Recall that $H \times \hat{V} \rightarrow \hat{V}$ is effective if only the identity element of $H$ fixes all points of $\hat{V}$.) 
Note that $\pi(H q)$, for $q \in \hat{V}$, gives the orbits of the isometries in the collapsed directions.

We say that $g_{M}$ is $\delta$-round if it is $\delta$-round at every $p \in M$.

The next theorem is a direct consequence of $[4,1.3]$. Let $\nabla$ denote the Levi-Civita connection for $g_{M}$ and let $\nabla^{\prime}$ denote the Levi-Civita connection for the Riemannian metric $g_{M}^{\prime}$ given in the following Theorem:

Theorem A.2.2 (Cheeger-Fukaya-Gromov). Given any $\epsilon>0$, any integer $m>0$, and any collection of positive numbers $A=\left\{A_{i} \mid i=0,1,2, \ldots\right\}$, there exists a positive number $\eta(\epsilon, m, A)$. For any $\delta \in(0, \eta(\epsilon, m, A))$, and for any $A$-regular complete Riemannian manifold $\left(M, g_{M}\right)$ of dimension $m$, there exists a $\delta$-round metric $g_{M}^{\prime}$ such that the following hold:

(a) $e^{-\epsilon} g_{M}<g_{M}^{\prime}<e^{\epsilon} g_{M}$.

(b) $\left|\nabla-\nabla^{\prime}\right|<\epsilon$.

(c) $M, g_{M}^{\prime}$ is $A^{\prime}$-regular, where $A^{\prime}=\left\{A_{i}^{\prime}: i=0,1,2,3, \ldots\right\}$ denotes a collection of positive numbers which depends only on $\epsilon, m$, and $A=$ $\left\{A_{i}: i=0,1,2,3, \ldots\right\}$. (In fact, $A_{i}^{\prime}$ depends only on $A_{0}, i, \epsilon$ and $m$.)

Note that for fixed $A=\left\{A_{i}\right\}$, there are sequences of $A$-regular manifolds whose injectivity radius approaches 0 ; so the cover of $V$ by $\hat{V}$ is nontrivial. Thus $\Lambda$ and $H$ are nontrivial as well and the collapsing can be seen.

In the next theorem we will need the following notation: Let $\rho: E \rightarrow \hat{V}$ denote the bundle of orthonormal frames over $\hat{V}$ with respect to the metric $g_{\hat{V}}^{\prime}$ (the pull back of $g_{M}^{\prime}$ along $\pi: \hat{V} \rightarrow V$ ). $E$ is given a Riemannian metric structure $g_{E}^{\prime}$ of bounded geometric type as follows: For any $v_{i} \in T E, i=1,2$, choose paths $\alpha_{i}:[-1,1] \rightarrow E, i=1,2$, such that $\dot{\alpha}_{i}(0)=v_{i}$ holds; each $\alpha_{i}$ may be regarded as a path $\beta_{i}:[-1,1] \rightarrow \hat{V}$ together with an orthonormal frame $\left(w_{i, 1}(t), w_{i, 2}(t), \ldots, w_{i, m}(t)\right)$ along $\beta_{i}(t), t \in[-1,1]$. Let $V_{i, j}$ denote the covariant derivative of $w_{i, j}(t)$ at $t=0$, and set

$$
g_{E}^{\prime}\left(v_{1}, v_{2}\right)=g_{\hat{V}}^{\prime}\left(\dot{\beta}_{1}(0), \dot{\beta}_{2}(0)\right)+\sum_{j=1}^{m} g_{\hat{V}}^{\prime}\left(V_{1, j}, V_{2, j}\right)
$$

Let $d_{E}^{\prime}($,$) denote the metric on E$ associated to $g_{E}^{\prime}$. Note that the canonical lifting $H \times E \rightarrow E$ of the group action $H \times \hat{V} \rightarrow \hat{V}$ is a free isometric group action (cf. A.2.1(c)). The following theorem is not explicitly stated in $[4]$, so we shall derive it at the end of this appendix.

Theorem A.2.3. There exists a real number $B>1$, which depends only on $n=\operatorname{dim} M, \epsilon$, and on the sectional curvature bound $A_{0}$ for $M$, such that the following is true. Suppose for $x \in E$ and $g \in H(g \neq e)$ we have that $d_{E}^{\prime}(x, g(x))<\delta$. Then there is a one-parameter subgroup $\phi: \mathbf{R} \rightarrow H$ of $H$ satisfying: 
(a) $\phi(t)=g$, for some $t \in(0, B \delta)$.

(b) The path $f: \mathbf{R} \rightarrow E$, defined by $f(s)=\phi(s)(x)$, has unit speed.

For any $y \in \hat{V}$ we set $H_{y}=\{h \in H: h(y)=y\}$, i.e., $H_{y}$ is the isotropy group at $y$ for the action $H \times \hat{V} \rightarrow \hat{V}$. Choose $\hat{y} \in \rho^{-1}(y)$. Since the lifted action $H \times E \rightarrow E$ is a free action, it follows from A.2.3 that the map $f: H \rightarrow E$ given by $f(g)=g(\hat{y})$ is an embedding onto a closed subset of $E$. Since $H_{y}$ is a closed subset of $H$ it follows that the restricted map $f: H_{y} \rightarrow \rho^{-1}(y)$ is an embedding onto a closed subset of the compact space $\rho^{-1}(y)$. Thus we have proven the following corollary of Theorem A.2.3:

Corollary A.2.4. For each $y \in \hat{V}$ the isotropy group $H_{y}$ is a compact subgroup of $H$.

We shall also need the following theorem, which among other uses will be used in proving Theorem A.2.3. Recall that the notion of "local angle control" was defined in 2.1 of $\S 2$ above.

Theorem A.2.5. The action $H \times E \rightarrow E$ has local angle control equal $(\lambda t, t)$ for any $t>0$, where $\lambda$ depends only on $\operatorname{dim} E, A_{0}, \epsilon$.

Remark A.2.6. In fact, we will show in proving A.2.5 that $\lambda$ depends only on $\operatorname{dim} \hat{V}$ and the sectional curvature bound $A_{0}^{\prime}$ for $\hat{V}$. Recall from A.2.2 that $A_{0}^{\prime}$ depends only on $A_{0}, \epsilon$ and $n=\operatorname{dim} \hat{V}$. Also, that $\operatorname{dim} E=n(n+1) / 2$. Moreover, we will use no special facts about $\hat{V}$; only that $H$ acts effectively on $\hat{V}$ by isometries.

Proof of Theorem A.2.5. Let $x \in E, u \in T E_{x}$ and $\alpha_{v}: \mathbf{R} \rightarrow H$ be a oneparameter subgroup with $\dot{\alpha}_{v}(0)=v \in \mathfrak{g}(H)$, where $\mathfrak{g}(H)$ is the Lie algebra of $H$. Define a curve $\gamma: \mathbf{R} \rightarrow E$ by

$$
\gamma(t)=\alpha_{v}(t) x
$$

and a vector field $\hat{u}$ along $\gamma$ by

$$
\hat{u}(t)=d \alpha_{v}(t)(u) .
$$

And let $\bar{D}$ denote the Levi-Civita connection on $E$. The argument above proving Lemma 2.3 yields that it suffices to show that $\left|\bar{D}_{\dot{\gamma}_{(0)}} \hat{u}\right|$ is bounded above by a constant multiplied by $|\dot{\gamma}(0) \| u|$ and this constant depends only on $n=\operatorname{dim} \hat{V}$ and $A_{0}^{\prime}$ (see Remark A.2.6). Since $\dot{\gamma}(0) \neq 0$ when $v \neq 0$, in bounding $\left|\bar{D}_{\dot{\gamma}(0)} \hat{u}\right|$, we may assume (and do assume) that $|u|=|\dot{\gamma}(0)|=1$ and need only consider the two cases: $u$ vertical and $u$ horizontal with 
respect to the Riemannian submersion

$$
O(n) \longrightarrow E \stackrel{\rho}{\longrightarrow} \hat{V}
$$

where $n=\operatorname{dim} \hat{V}$.

Let us first consider the case where $u$ is vertical. A continuity argument then shows that we need only consider the subcase where $\rho \circ \gamma: \mathbf{R} \rightarrow \hat{V}$ is an immersion. To verify this subcase, we need the following lemma. Consider the submanifold $S$ of $E$ defined by

$$
S=\rho^{-1}(\operatorname{image}(\rho \circ \gamma)) \text {. }
$$

Lemma A.2.5.1. Let $B($,$) denote the second fundamental form of S$ in $E$. The set $\{|B(v, u)|:|u|,|v| \leq 1$ and $u$ vertical $\}$ is bounded above by a positive real number which depends only on $n$ and $A_{0}^{\prime}$.

Before proving Lemma A.2.5.1, we use it to bound $\left|\bar{D}_{\dot{\gamma}(0)} \hat{u}\right|$ when $u$ is vertical. Note that the map

$$
\zeta: \mathbf{R} \times O(n) \rightarrow S
$$

defined by

$$
(t, g) \rightarrow \alpha_{v}(t) x g^{-1}=\gamma(t) g^{-1}
$$

is either a bijective immersion or a covering projection depending on whether $\rho \circ \gamma$ is monic or not. Use this map to put a left invariant Riemannian metric on $\mathbf{R} \times O(n)$; in particular, an inner product $\langle$,$\rangle on the Lie algebra$ $\mathfrak{g}(\mathbf{R} \times O(n))$. Since $\langle$,$\rangle restricted to \mathfrak{g}(O(n))$ is -1 times the Killing form, it satisfies the equation:

\section{A.2.5.2. $\langle[a, b], a\rangle=0$}

valid for all $a, b \in \mathfrak{g}(O(n))$.

Remark A.2.5.2.1. When $n=1$ or 2 , instead of the negative of the Killing form, we mean the unique bi-invariant metric such that $\operatorname{Vol}(O(n))=2$ or $4 \pi$, respectively.

Let $U, V \in \mathfrak{g}(\mathbf{R} \times O(n))$ be the vectors corresponding to $u, \dot{\gamma}(0) \in T E_{x}$ via $d \zeta$. And let $\hat{U}, \hat{V}$ be left invariant vector fields on $\mathbf{R} \times O(n)$ determined by $U, V$. Then note that

$$
\bar{D}_{\dot{\gamma}(0)} \hat{u}=d \zeta\left(D_{V} \hat{U}\right)+B(\dot{\gamma}(0), u)
$$

where $D$ is the Levi-Civita connection for $\mathbf{R} \times O(n)$ and $B($,$) is the second$ fundamental form of $S$ in $E$. Because of this equation and Lemma A.2.5.1, it remains to show the following to verify A.2.5 when $u$ is vertical: 
Lemma A.2.5.3. Let $T$ and $X$ be unit length left invariant vector fields on $\mathbf{R} \times O(n)$ tangent to $\mathbf{R} \times *$ and $* \times O(n)$, respectively. Then $\left|D_{T} X\right|$ is bounded above by a constant which depends only on $n$.

Proof of Lemma A.2.5.3. Identify the left invariant vector fields on $\mathbf{R} \times O(n)$ with the elements in its Lie algebra $\mathfrak{g}(\mathbf{R} \times O(n))$. Then orthogonally decompose $T$ and $D_{T} X$ as:

A.2.5.3.1. $D_{T} X=Y+R$ and $T=Z+F$

where $Y, Z \in \mathfrak{g}(O(n))$ and $R, F \in \mathfrak{g}(O(n))^{\perp}$. Recall [2, Prop. 7.7.1] which states that:

A.2.5.3.2. $\left\langle D_{a} b, c\right\rangle=1 / 2(\langle[b, a], c\rangle+\langle[a, c], b\rangle+\langle[b, c], a\rangle)$

for all $a, b, c \in \mathfrak{g}(\mathbf{R} \times O(n))$. And note that:

A.2.5.3.3. $[T, a]=0$

for all $a \in \mathfrak{g}(O(n))$. Setting $a=T, b=X$, and $c=Y$ in A.2.5.3.2 and applying A.2.5.3.3, we obtain:

A.2.5.3.4. $\left\langle D_{T} X, Y\right\rangle=1 / 2\langle[X, Y], T\rangle$.

Note also that the norm of the Lie bracket [, ] restricted to $\mathfrak{g}(O(n))$ is bounded above by a constant $\mathbf{r} \geq 0$ which depends only on $n$ since $\langle$, $\rangle$ restricted to $\mathfrak{g}(O(n))$ is the negative of the Killing form; cf. Remark A.2.5.2.1. Consequently, A.2.5.3.1 and A.2.5.3.4 yield that:

A.2.5.3.5. $|Y| \leq \mathbf{r} / 2$.

Next, setting $a=T, b=X$, and $c=T$ in A.2.5.3.2 and applying A.2.5.3.3, we obtain:

A.2.5.3.6. $\left\langle D_{T} X, T\right\rangle=0$.

Hence A.2.5.3.1 and A.2.5.3.6 yield:

A.2.5.3.7. $\left\langle D_{T} X, F\right\rangle=-\left\langle D_{T} X, Z\right\rangle$.

Setting $a=T, b=X$, and $c=Z$ in A.2.5.3.2 and applying A.2.5.3.3 yields:

A.2.5.3.8. $\left\langle D_{T} X, Z\right\rangle=(1 / 2)\langle[X, Z], T\rangle$.

And substituting Equation A.2.5.3.1 for $T$ into A.2.5.3.8 yields:

A.2.5.3.9. $\left\langle D_{T} X, Z\right\rangle=(1 / 2)\langle[X, Z], F\rangle$

since $Z \perp[X, Z]$ by A.2.5.2 above. Combining A.2.5.3.7 and A.2.5.3.9 yields: 
A.2.5.3.10. $\left\langle D_{T} X, F\right\rangle=-(1 / 2)\langle[X, Z], F\rangle$.

Note that $F \neq 0$ since we assumed that $\rho \circ \gamma: \mathbf{R} \rightarrow \hat{V}$ is an immersion. Therefore, $R$ is a scalar multiple of $F$ and A.2.5.3.10 consequently yields:

A.2.5.3.11. $\left\langle D_{T} X, R\right\rangle=-(1 / 2)\langle[X, Z], R\rangle$.

Now A.2.5.3.11 implies:

A.2.5.3.12. $|R| \leq \mathbf{r} / 2$

in the same way that A.2.5.3.4 implies A.2.5.3.5. Combining the inequalities of A.2.5.3.12 and A.2.5.3.5 yields that $\left|D_{T} X\right| \leq \mathbf{r}$ proving Lemma A.2.5.3.

To prove Lemma A.2.5.1 we need to calculate $\bar{D}$. And for this purpose we define special horizontal and vertical vector fields on $E$ as follows: A horizontal vector field $\bar{X}$ on $E$ is special if there exists a vector field $X$ on $\hat{V}$ such that

$$
d \rho\left(\bar{X}_{x}\right)=X_{\rho(x)}
$$

for all $x \in E$. Let $\mathfrak{g}(O(n))$ denote the Lie algebra of $O(n)$. Each $v \in \mathfrak{g}(O(n))$ determines a special vertical vector field $\check{v}$ on $E$ by requiring

$$
\check{v}_{x}=\dot{\delta}_{x}(0)
$$

for each $x \in E$. Here, $\delta_{x}(t)=x \beta_{v}(t)$ where $\beta_{v}$ is the one-parameter subgroup of $O(n)$ such that $\dot{\beta}_{v}(0)=v$. To calculate $\bar{D}$ it suffices to calculate $\left(\bar{D}_{a} b\right) \cdot c$, where $a, b, c$ are the special vector fields just defined. A routine application of the Koszul formula yields the following result:

Lemma A.2.5.4. Let $u, v, w \in \mathfrak{g}(O(n))$ and $X, Y, Z$ be vector fields on $\hat{V}$. Also let $\mathcal{B}(, \quad)$ denote the negative of the Killing form on $\mathfrak{g}(O(n))$ and $D$ denote the Levi-Civita connection on $\hat{V}$; cf. Remark A.2.5.2.1. Then the following are valid equations:

(a) $\left(\bar{D}_{\check{u}} \check{v}\right) \cdot \check{w}=(1 / 2) \mathcal{B}([u, v], w)$;

(b) $\left(\bar{D}_{\bar{X}} \bar{Y}\right) \cdot \bar{Z}=\left(D_{X} Y\right) \cdot Z$;

(c) $\left(\bar{D}_{\breve{u}} \bar{X}\right) \cdot \bar{Y}=\left(\bar{D}_{\bar{X}} \check{u}\right) \cdot \bar{Y}=-(\bar{D} \bar{X} \bar{Y}) \cdot \check{u}=(1 / 2)[\bar{Y}, \bar{X}] \cdot \check{u}$;

(d) $\left(\bar{D}_{\check{u}} \check{v}\right) \cdot \bar{X}=\left(\bar{D}_{\bar{X}} \check{v}\right) \cdot \check{u}=\left(\bar{D}_{\check{u}} \bar{X}\right) \cdot \check{v}=0$.

Proof of Lemma A.2.5.1. It suffices to prove the assertion in the two cases: $v$ vertical and $v$ horizontal. The equation of A.2.5.4(d) shows that the fibers of $\rho$ are totally geodesic submanifolds of $E$; i.e., $B(v, u)=0$ when both $u$ and $v$ are vertical. Therefore, it remains to estimate

$$
B(\bar{X}, u) \cdot \bar{Y}
$$


where $\bar{X}$ and $\bar{Y}$ are special horizontal vector fields on $E$ induced by vector fields $X$ and $Y$, respectively, on $\hat{V}$. (Also, $\bar{X}$ is assumed tangent to $S$ and $\bar{Y}$ perpendicular to $S$ at points of $S$.) In this situation, the equation of A.2.5.4(c) yields that

$$
B(\bar{X}, u) \cdot \bar{Y}=1 / 2[\bar{Y}, \bar{X}] \cdot u=\Omega(\bar{X}, \bar{Y}) \cdot w
$$

where $\Omega($,$) denotes the curvature form for the frame bundle of \hat{V}$ and $w$ is the unique vector in $\mathfrak{g}(O(n))$ such that $\check{w}_{x}=u$. Since the norm of the curvature tensor for $\hat{V}$ is bounded by $A_{0}^{\prime}$, we also get the desired upper bound for $|B(v, u)|$ in this case. (See [15, Vol. 1, p. 133] for the standard relation between $\Omega($,$) and the curvature tensor on \hat{V}$.)

This completes the proof for Lemma A.2.5.1.

It remains, in proving Theorem A.2.5, to consider the case where $u$ is horizontal with respect to $\rho$. Since we have assumed that $|\dot{\gamma}(0)|=|u|=1$, it suffices to show that $\left|\bar{D}_{\dot{\gamma}(0)} \hat{u}\right|$ is bounded above by a real number which depends only on $n$ and $A_{0}^{\prime}$. Let $z=\rho(x)$ and define a curve $\beta(t)$ in $\hat{V}$ by

$$
\beta(t)=\alpha_{v}(t) z \text {. }
$$

And note that $\beta=\rho \circ \gamma$. Decompose

$$
\dot{\gamma}(0)=v^{+}+v^{-}
$$

where $v^{+}$is vertical and $v^{-}$is horizontal relative to $\rho$. A continuity argument yields that it suffices to consider the situation where $v^{-} \neq 0$; hence, we now make this assumption. Also, note that

$$
\left|v^{+}\right|,\left|v^{-}\right| \leq 1 \text {. }
$$

Since $\dot{\beta}(0)=d \rho\left(v^{-}\right) \neq 0$, there is a vector field $Y$ on $\hat{V}$ such that the induced special horizontal vector field $\bar{Y}$ on $E$ satisfies

$$
\bar{Y}_{\gamma(t)}=\hat{u}(t)
$$

for all $t$ sufficiently close to 0 . Hence, it suffices to show that $\left|\bar{D}_{v^{+}} \bar{Y}\right|$ and $\left|\bar{D}_{v^{-}} \bar{Y}\right|$ are both bounded above by a real number which depends only on $n, A_{0}$, and $\epsilon$. Therefore it remains to estimate

$$
\left(\bar{D}_{v^{ \pm}} \bar{Y}\right) \cdot \bar{Z} \text { and }\left(\bar{D}_{v^{ \pm}} \bar{Y}\right) \cdot \check{w}
$$

where $Z$ is a vector field on $\hat{V}$ with $\left|Z_{\beta(0)}\right|=1$ and $w \in \mathfrak{g}(O(n))$ with $|w|=1$. (Recall $\check{w}$ is the special vertical vector field induced by $w$.) We will use Lemma A.2.5.4 to do these estimates. For this purpose, we need to fix some notation. Let $X$ be a vector field on $\hat{V}$ such that $X_{\beta(0)}=d \rho\left(v^{-}\right)$. (Note that $\bar{X}_{\gamma(0)}=v^{-}$.) Also, let $\omega \in \mathfrak{g}(O(n))$ be the vector satisfying

$$
\check{\omega}_{\gamma(0)}=v^{+} \text {. }
$$


Let $u_{0}=d \rho(u)$ and $\hat{u}_{0}$ denote the vector field along the curve $\beta$ in $\hat{V}$ defined by

$$
\hat{u}_{0}(t)=d \alpha_{v}(t)\left(u_{0}\right)
$$

Recall that $D$ is the covariant derivative in $\hat{V}$. And $\Omega($, ) denotes (as before) the curvature form for the frame bundle of $\hat{V}$. With this notation, Lemma A.2.5.4 yields the following calculation:

A.2.5.5. (a) $\left(\bar{D}_{v^{+}} \bar{Y}\right) \cdot \check{w}=0$;

(b) $\left(\bar{D}_{v^{-}} \bar{Y}\right) \cdot \check{w}=(1 / 2)[\bar{X}, \bar{Y}] \cdot \check{w}=\Omega(\bar{Y}, \bar{X}) \cdot \check{w}$;

(c) $\left(\bar{D}_{v^{+}} \bar{Y}\right) \cdot \bar{Z}=(1 / 2)[\bar{Z}, \bar{Y}] \cdot \check{\omega}=\Omega(\bar{Y}, \bar{Z}) \cdot \check{\omega}$;

(d) $\left(\bar{D}_{v^{-}} \bar{Y}\right) \cdot \bar{Z}=\left(D_{X} Y\right) \cdot Z=D_{\beta(0)} \hat{u}_{0} \cdot Z$.

The four equations of A.2.5.5(a)-(d) combined with the fact that the norm of the curvature tensor for $\hat{V}$ is bounded by $A_{0}^{\prime}$ yield the necessary estimates for $\left|\bar{D}_{v^{+}} \bar{Y}\right|$ and $\left|\bar{D}_{v^{-}} \bar{Y}\right|$ completing the proof for Theorem A.2.5 once we have verified the following lemma. (See again [15, Vol. 1, p. 133] for the standard relation between $\Omega($,$) and the curvature tensor on \hat{V}$. It yields the bound for the norm of $\Omega($, ) needed for using A.2.5.5(b) and (c).)

Lemma A.2.5.6. $\left|D_{\beta(0)} \hat{u}_{0} \cdot Z\right| \leq n$, where $n=\operatorname{dim} \hat{V}$.

Proof of Lemma A.2.5.6. Recall that $\gamma(0)$ is an orthonormal framing for the tangent space of $\hat{V}$ at $\beta(0)$; i.e., $\gamma(0)=\left(u_{1}, u_{2}, \ldots, u_{n}\right)$ where each $u_{i} \in$ $T_{\beta(0)} \hat{V}$ and

$$
u_{i} \cdot u_{j}= \begin{cases}0 & \text { if } i \neq j \\ 1 & \text { if } i=j\end{cases}
$$

And notice that $\gamma(t)$ is the frame field $\left(\hat{u}_{1}(t), \hat{u}_{2}(t), \ldots, \hat{u}_{n}(t)\right)$ along the curve $\beta(t)$ in $\hat{V}$; consequently,

$$
|\dot{\gamma}(0)|^{2}=|\dot{\beta}(0)|^{2}+\left|D_{\dot{\beta}(0)} \hat{u}_{1}\right|^{2}+\cdots+\left|D_{\dot{\beta}(0)} \hat{u}_{n}\right|^{2} .
$$

We conclude from (1) and $|\dot{\gamma}(0)|=1$ that

$$
\left|D_{\dot{\beta}_{(0)}} \hat{u}_{i}\right| \leq 1 \text { for } i=1,2, \ldots, n .
$$

Express $u_{0} \in T_{\beta(0)} \hat{V}$ in terms of the orthonormal frame $\left(u_{1}, u_{2}, \ldots, u_{n}\right)$; i.e., let $u_{0}=a_{1} u_{1}+a_{2} u_{2}+\cdots+a_{n} u_{n}$. Then for each $i$ we have that

$$
\left|a_{i}\right| \leq 1
$$


since $\left|u_{0}\right|=1$. Note also that $\hat{u}_{0}=a_{1} \hat{u}_{1}+a_{2} \hat{u}_{2}+\ldots+a_{n} \hat{u}_{n}$. It follows therefore from inequalities (2) and (3) that

$$
\left|D_{\beta(0)} \hat{u}_{0}\right| \leq \sum_{i=1}^{n}\left|a_{i}\right|\left|D_{\beta(0)} \hat{u}_{i}\right| \leq n .
$$

The inequality posited in the lemma now follows immediately from (4) and the fact that $|Z| \leq 1$.

This completes the proof of Theorem A.2.5.

Proof of Theorem A.2.3. We may identify $\hat{V}$ with $T_{R}(H y)$; i.e., with the tubular neighborhood of radius $R>\delta$ where $y \in \hat{V}$ is a point such that the isotropy subgroup $C$ of the action of $H$ at $y$ is a maximal compact subgroup of the virtually nilpotent Lie group $H$; cf. $[4, \S 8]$.

(We must distinguish between the orbit of $y$ under the action of $H$ denoted by $H y$ and the isotropy subgroup of $H$ fixing $y$ denoted by $H_{y}$.)

Remark 1. Let $\bar{y} \in F \hat{V}=E$ lie over $y$; i.e., $\rho(\bar{y})=y$ where $\rho: E \rightarrow \hat{V}$ is the canonical projection. Then the equation

$$
c \bar{y}=\bar{y} \bar{c}, \text { where } c \in C,
$$

identifies $C$ with a subgroup $\bar{C}$ of $O(n), n=\operatorname{dim} \hat{V}$. (Throughout the proof, $n$ denotes $\operatorname{dim} \hat{V}=\operatorname{dim} M$.) In fact, $\bar{C}$ is the isotropy group of the (right) action of $O(n)$ on $H \backslash E$ at $H \bar{y}$. Consequently, $\bar{C}$ is a maximal isotropy group for the action of $O(n)$ on $H \backslash E$.

Fact 1. There exists a positive integer $\bar{k}=\bar{k}\left(n, \epsilon, A_{0}\right)$ and closed subgroups $C_{1}, C_{2}, \ldots, C_{\bar{k}}$ of $O(n)$, depending only on $n, \epsilon$ and $A_{0}$, such that $\bar{C}$ is orthogonally conjugate to some group $C_{i}$ in this list.

Fact 1 is verified at the end of this appendix after using it to prove Theorem A.2.3. There are the following ten steps in this Proof of Theorem A.2.3:

Step 1. Let $\alpha:[0,1] \rightarrow E=F \hat{V}$ be a piecewise smooth path connecting $x$ to $g x$ such that length $(\alpha)<\delta$. Let $\psi: \hat{V}=T_{R}(H y) \rightarrow H y$ be the orthogonal projection. It clearly suffices to consider the case where $\psi(\rho(x))=y$. So we make this assumption. Let $\beta:[0,1] \rightarrow H y$ be the composite of $\alpha$ and $\psi \circ \rho$; i.e.,

$$
\beta=\psi \circ \rho \circ \alpha
$$

Claim. There exists a constant $\mathcal{C}_{1}=\mathcal{C}_{1}\left(n, \epsilon, A_{0}\right)>0$ such that length $(\beta)<$ $\mathcal{C}_{1} \delta$ where $H y$ has the Riemannian metric determined by being a submanifold of $\hat{V}$. 
Step 2. There exists a piecewise smooth path $\bar{\beta}:[0,1] \rightarrow H$ and a constant $\mathcal{C}_{2}=\mathcal{C}_{2}\left(n, \epsilon, A_{0}\right)$ satisfying the following properties where $\hat{\beta}:[0,1] \rightarrow E$ is defined by $\hat{\beta}(t)=\bar{\beta}(t) \bar{y}$ for all $t \in[0,1]$ :

1. $\bar{\beta}(0)=e$. (Here $e$ is the identity element of $H$.)

2. $\rho \circ \hat{\beta}=\beta$.

3. length $(\hat{\beta})<\mathcal{C}_{2} \delta$.

Step 3. Define a piecewise smooth path $\gamma:[0,1] \rightarrow E$ by $\gamma(t)=\bar{\beta}(t) x$ for all $t \in[0,1]$. It clearly satisfies the following properties:

1. $\gamma(0)=x$.

2. $\psi \circ \rho \circ \gamma=\beta$.

Claim. There exists a constant $\mathcal{C}_{3}=\mathcal{C}_{3}\left(n, \epsilon, A_{0}\right)>0$ such that length $(\gamma)<$ $\mathcal{C}_{3} \delta$.

Step 4. Let $h=\bar{\beta}(1) \in H$. Because of Step 3 (Property 2), we have $h y=g y$ and consequently $y=\left(h^{-1} g\right) y$. Thus setting $c=h^{-1} g$, we have that $c \in C$. Also

$$
d_{E}(x, c x) \leq\left(\mathcal{C}_{3}+1\right) \delta
$$

because of the following reasoning:

$$
d_{E}(x, c x)=d_{E}\left(x, h^{-1} g x\right)=d_{E}(h x, g x) \leq d_{E}(h x, x)+d_{E}(x, g x) \leq \mathcal{C}_{3} \delta+\delta .
$$

Claim. There exists a constant $\mathcal{C}_{4}=\mathcal{C}_{4}\left(n, \epsilon, A_{0}\right)>0$ such that $d_{E}(\bar{y}, c \bar{y})<$ $\mathcal{C}_{4} \delta$

Remark. Let $\Psi: E \rightarrow \rho^{-1}(H y)=H \bar{y} O(n)$ be the orthogonal projection. Then $\Psi$ is $H, O(n)$ bi-equivariant and covers $\psi: \hat{V} \rightarrow H y$. Also fix $\bar{y}=\Psi(x)$; i.e., this is a specific framing of $y$. Then the above claim will result by establishing the inequality

$$
d_{E}(\Psi(x), \Psi(c x)) \leq \mathcal{C}_{4} d_{E}(x, c x) .
$$

Step 5. We make the following claim in this step:

Claim. There exists a constant $\mathcal{C}_{5}=\mathcal{C}_{5}\left(n, \epsilon, A_{0}\right)>0$ such that $d_{\rho^{-1}(y)}(\bar{y}, c \bar{y})$ $<\mathcal{C}_{5} \delta$.

Remark. This is proved using the facts that:

1. $\rho^{-1}(y)$ is a totally geodesic submanifold of $E=F \hat{V}$;

2. $\rho: E \rightarrow \hat{V}$ is a Riemannian submersion;

3. $\hat{V}$ has injectivity radius $>\delta$ at $y$.

Step 6. In this step we make the following claim: 
Claim. Provided $\delta$ is sufficiently small, then $d_{C}(\bar{y}, c \bar{y})<2 \mathcal{C}_{5} \delta$.

Remark. Recall that $c \bar{y}=\bar{y} \bar{c}$ where $\bar{c} \in \bar{C} \subset O(n)$. (See Remark 1 at the beginning of this proof.) Note also, for each $z \in E$, that the orbit map $g \rightarrow z g$ is an isometry between $O(n)$ with its standard metric and $z O(n)$ with its Riemannian metric induced by the inclusion $z O(n) \subset E$. Then the above claim is a consequence of Fact 1 (at the beginning of this proof) and the Claim made in Step 5.

Step 7. The following inequality is an immediate consequence of the Claim made in Step 6 and the inclusion $C \bar{y} \subset H \bar{y}$ :

$$
d_{H \bar{y}}(\bar{y}, c \bar{y})<2 \mathcal{C}_{5} \delta .
$$

Step 8. We have the following claim:

Claim. $d_{H x}(x, c x)<\mathcal{C}_{3}\left(2 \mathcal{C}_{5}\right) \delta$.

This claim is a consequence of the inequality posited in Step 7 together with the argument which yields the Claim made in Step 3.

Step 9 . The triangle inequality says that

$$
d_{H x}(x, g x) \leq d_{H x}(x, h x)+d_{H x}(h x, g x)=d_{H x}(x, h x)+d_{H x}(x, c x) .
$$

Combining this inequality with the claims made in Steps 3 and 8 yields that

$$
d_{H x}(x, g x)<\mathcal{C}_{3}\left(1+2 \mathcal{C}_{5}\right) \delta .
$$

Step 10. Put on $H$ the left invariant Riemannian metric induced from the monic immersion $H \rightarrow E$ defined by $k \rightarrow k x$ for all $k \in H$. Then the sectional curvatures of $H$ are bounded above by a constant which depends only on $n, \epsilon$ and $A_{0}$. This fact is a consequence of Theorem A.2.5. Hence, provided $\delta$ is sufficiently small (how small again depends only on $n, \epsilon$ and $A_{0}$ ) we can use Claim 2.8.5 (from $\S 2$ ) in conjunction with the inequality demonstrated in Step 9 to produce a 1-parameter subgroup $\phi: \mathbf{R} \rightarrow G$ satisfying Properties (a) and (b) posited in Theorem A.2.3 with $B=4^{n} \mathcal{C}_{3}\left(1+2 \mathcal{C}_{5}\right)$.

This completes the outline of the main steps in the Proof of Theorem A.2.3. Now in the following remarks we shall indicate some more details in the verifications of the Claims made in the preceding steps.

\section{Remarks on Step 10.}

To show that the hypotheses of Claim 2.8.5 are satisfied use the following Lemma which we proceed now to formulate and prove:

Let $G$ be a simply connected nilpotent Lie group with Lie algebra $\mathfrak{g}$ equipped with an inner product. Put on $G$ the corresponding left invariant 
Riemannian metric. Let $\mathbf{a}_{1}$ denote the norm of the Lie bracket on $\mathfrak{g}$; i.e.,

$$
\mathbf{a}_{1}=\max \{|[u, v]|: u, v \in \mathfrak{g} \text { and }|u|=1=|v|\} .
$$

Let $\mathbf{a}_{2}$ denote the square root of the maximum of the absolute values of the sectional curvatures of $G$. And let $\mathbf{a}_{3}$ denote the norm of $D_{\hat{v}} \hat{u}$; i.e.,

$$
\mathbf{a}_{3}=\max \left\{\left|D_{\hat{v}} \hat{u}\right|: u, v \in \mathfrak{g} \text { and }|u|=1=|v|\right\},
$$

where $\hat{u}, \hat{v}$ are the left invariant vector fields on $G$ with $\hat{u}(e)$ and $\hat{v}(e)=v$. (Here $e$ is the identity element of $G$.)

Lemma. There is a positive real number $B$ which depends only on $\operatorname{dim} G$, such that $\mathbf{a}_{i} \leq B \mathbf{a}_{j}$ for all $i, j \in\{1,2,3\}$.

Proof of Lemma. From [2, Proposition 7.7.1] one deduces the following two inequalities:

(a) $\mathbf{a}_{2} \leq \sqrt{6} \mathbf{a}_{1}$;

(b) $\mathbf{a}_{3} \leq(3 / 2) \mathbf{a}_{1}$.

Using [16, Theorem 47, p. 213], one can construct a positive real number $\bar{B}$, which depends only on $\operatorname{dim} G$, such that:

(c) $\mathbf{a}_{1} \leq \bar{B} \mathbf{a}_{2}$.

Also Claims 2.8.1 and 2.8.2 (proven in $\S 2$ above) yield the following inequality:

(d) $\mathbf{a}_{1} \leq 2(\operatorname{dim} G)^{2} \mathbf{a}_{3}$.

Concatenating inequalities (a)-(d) proves the Lemma.

\section{Remarks on Steps 1 and 4.}

Inequality (8.7) of [4] states that the norm of the second fundamental form of $H y$ in $\hat{V}$ is bounded above by a positive constant depending only on $n, \epsilon$ and $A_{0}$. (This can also be independently verified using that the angle between $H \bar{y}$ and $\bar{y} O(n)$ is bounded below. We will show this in "Remarks on Step 2" given below.) Then Lemma A.2.5.4 can be used to verify the same fact for the second fundamental form of $H \bar{y} O(n)$ in $E$. Consequently, both $\psi$ and $\Psi$ are $\Theta$-almost Riemannian submersions (cf. 2.5.1 of [4] for the definition) where $\Theta$ depends only on $n, \epsilon$ and $A_{0}$.

\section{Remarks on Steps 3 and 8.}

Note that $\Psi \circ \gamma=\hat{\beta}$ and recall from "Remarks on Steps 1 and 4" that $\Psi$ is a $\Theta$-almost Riemannian submersion where $\Theta$ depends only on $n, \epsilon$ and $A_{0}$. Also note that $\gamma$ is tangent to the foliation of $E$ by the orbits of $H$. So it suffices to show that the angle between $H x$ and $\Psi^{-1}(\bar{y})$ at $x$ is larger than $\mathcal{C}_{6}=\mathcal{C}_{6}\left(n, \epsilon, A_{0}\right)>0$. But because of Theorem A.2.5, we can apply Lemma 2.6 to conclude this. Step 8 follows by the same argument. 


\section{Remarks on Step 2.}

Let $\bar{\rho}: H \bar{y} \rightarrow H y$ be the restriction of $\rho: F \hat{V} \rightarrow \hat{V}$. (Recall $\rho(\bar{y})=y$ and $\rho$ is $H$-equivariant.) It suffices to show that $\bar{\rho}$ is a $\Theta$-almost Riemannian submersion where $\Theta=\Theta\left(n, \epsilon, A_{0}\right)>0$. To do this, it is enough to show that the angle between the tangent spaces to $H \bar{y}$ and $\bar{y} O(n)$ at $\bar{y}$ is bounded below by a number $\bar{\Theta}=\bar{\Theta}\left(n, \epsilon, A_{0}\right)>0$. This is because $\rho: \rho^{-1}(H y)=$ $H \bar{y} O(n) \rightarrow H y$ is a Riemannian submersion.

Let $\mathcal{E}$ be the Riemannian manifold used in $[4, \S 8]$ to construct $\hat{V}$. There is a virtually nilpotent Lie group $\hat{H}$ such that $\hat{H}$ and $O(n)$ both act freely (and properly) by isometries on $\mathcal{E}$, these actions commute, and $\hat{V}=\mathcal{E} / O(n)$. ( $\hat{H}$ acts on the left and $O(n)$ on the right of $\mathcal{E}$.) Furthermore, there is a Lie group epimorphism $\phi: \hat{H} \rightarrow H$ with finite kernel such that $\left.\phi\right|_{\hat{H}^{0}}$ is an isomorphism of $(\hat{H})^{0}$ to $H^{0}$ and such that the principal $O(n)$-bundle projection

$$
\hat{\rho}: \mathcal{E} \rightarrow \hat{V}
$$

is $\phi$-equivariant; $\left[4\right.$, pp. 365, 369]. Let $\hat{C}=\phi^{-1}(C)$; it is a maximal compact subgroup of $\hat{H}$ and $\left.\phi\right|_{\hat{C}^{0}}$ maps $(\hat{C})^{0}$ isomorphically to $C^{0}$. We identify $(\hat{H})^{0}$ and $(\hat{C})^{0}$ with $H^{0}$ and $C^{0}$, respectively, via $\phi$. (Recall $G^{0}$ denotes the connected component of $G$ containing the identity element.)

Let $\hat{y} \in \mathcal{E}$ be a point such that $\hat{\rho}(\hat{y})=y$. To show the bound $\bar{\Theta}$ exists, we need the following result:

Theorem 1. The angle between the tangent spaces to $H^{0} \hat{y}$ and $\hat{y} O(n)$ at $\hat{y}$ is bounded below by a number $\hat{\Theta}=\hat{\Theta}\left(n, \epsilon, A_{0}\right)>0$.

To prove this result we need three lemmas.

Lemma 1. Given $\sigma>0$, there exists $\mu=\mu(\sigma, n)>1$ such that for any unit speed 1-parameter subgroup $\alpha: \mathbf{R} \rightarrow O(n)$ there is a number $t \in[1, \mu]$ with $d(\alpha(t), \alpha(0))<\sigma$.

Proof. There is a maximal torus $T$ in $O(n)$ containing the image of $\alpha$. The volume of $T$ depends only on $n$ since all maximal tori in $O(n)$ are conjugate. Consider the set of open balls in $T: B_{\sigma / 2}(\alpha(1)), B_{\sigma / 2}(\alpha(2)), B_{\sigma / 2}(\alpha(3)), \ldots$ They all have the same volume $V=V(n, \sigma)>0$. Let $\mu=1+[\operatorname{Vol}(T) / V]$. Then clearly there are two integers $i$ and $j$ with $1 \leq i<j \leq \mu$ such that

$$
B_{\sigma / 2}(\alpha(i)) \cap B_{\sigma / 2}(\alpha(j)) \neq \phi .
$$

Let $t=j-i$, then we are done with the proof of Lemma 1 .

Consider the orbit action of $\hat{H} \times O(n)$ on $\mathcal{E}$ at $\hat{y}$ defined by

$$
(a, b) \rightarrow a \hat{y} g^{-1}, \quad \text { where }(a, g) \in \hat{H} \times O(n)
$$


and denote this action by $(a, g) \cdot \hat{y}$. Since the induced actions of $\hat{H}$ and $O(n)$, respectively, are faithful, they determine left invariant Riemannian metrics on $\hat{H}$ and $O(n)$. The metric determined thusly on $O(n)$ is the same as that induced by the negative of the Killing form on $\mathfrak{g}(O(n))$; cf. Remark A.2.5.2.1. For any $v \in \mathfrak{g}\left(H^{0} \times O(n)\right)$, let $\alpha_{v}: \mathbf{R} \rightarrow H^{0} \times O(n)$ denote the corresponding 1-parameter subgroup. Let $u \in \mathfrak{g}(O(n))$ and $v \in \mathfrak{g}\left(H^{0}\right)$ be such that the two curves in $\mathcal{E}, \beta_{u}$ and $\beta_{v}$, defined by

$$
t \rightarrow \hat{y} \alpha_{-u}(t) \text { and } t \rightarrow \alpha_{v}(t) \hat{y}
$$

are both perpendicular to the orbit space $C^{0} \hat{y}$ at $\hat{y}$. Let $V \subset \mathfrak{g}\left(H^{0} \times O(n)\right)$ denote the subspace spanned by $u$ and $v$. Since $[u, v]=0$, this subspace is an abelian Lie subalgebra. Furthermore, assume that

$$
|u|=\left|\dot{\beta}_{u}(0)\right|=1
$$

and that when $\dot{\beta}_{v}(0)$ is orthogonally projected onto $T_{\hat{y}}(\hat{y} O(n))$ it hits the vector $\dot{\beta}_{u}(0)$. Denote the angle between $\dot{\beta}_{u}(0)$ and $\dot{\beta}_{v}(0)$ by $\Theta$.

Lemma 2. Given $\sigma>0$ there exists $t \geq 1$ such that

$$
d_{\mathcal{E}}\left(\alpha_{v}(t) \hat{y}, \hat{y}\right) \leq \sigma / 2+\mu(\sigma / 2, n) \tan (\Theta)
$$

and

$$
d_{H^{0} \hat{y}}\left(\alpha_{v}(t) \hat{y}, \hat{y}\right) \geq d=d\left(n, \epsilon, A_{0}\right)>0 .
$$

Proof. Let $t \in[1, \mu(\sigma / 2, n)]$ be the number posited in Lemma 1 when $\alpha=$ $\alpha_{-u}$. And let $w=v-u$, then $w \in V$. Hence we have that

$$
\alpha_{v}(t)=\alpha_{u}(t) \alpha_{w}(t)
$$

since $V$ is abelian; consequently

$$
d_{\mathcal{E}}\left(\alpha_{v}(t) \cdot \hat{y}, \hat{y}\right) \leq d_{\mathcal{E}}\left(\left(\alpha_{u}(t) \alpha_{w}(t)\right) \cdot \hat{y}, \alpha_{u}(t) \cdot \hat{y}\right)+d_{\mathcal{E}}\left(\alpha_{u}(t) \cdot \hat{y}, \hat{y}\right)
$$

by the triangle inequality. Substituting the equations

$$
\begin{aligned}
d_{\mathcal{E}}\left(\left(\alpha_{u}(t) \alpha_{w}(t)\right) \cdot \hat{y}, \alpha_{u}(t) \cdot \hat{y}\right) & =d_{\mathcal{E}}\left(\alpha_{w}(t) \cdot \hat{y}, \hat{y}\right) \text { and } \\
d_{\mathcal{E}}\left(\alpha_{u}(t) \cdot \hat{y}, \hat{y}\right) & =d_{O(n)}\left(\alpha_{-u}(t), \alpha_{-u}(0)\right)
\end{aligned}
$$

into (1) and applying Lemma 1 to $\alpha=\alpha_{-u}$, we obtain that

$$
d_{\mathcal{E}}\left(\alpha_{v}(t) \cdot \hat{y}, \hat{y}\right) \leq \sigma / 2+d_{\mathcal{E}}\left(\alpha_{w}(t) \cdot \hat{y}, \hat{y}\right) .
$$

Let $\beta_{w}$ denote the curve in $\mathcal{E}$ defined by

$$
s \rightarrow \alpha_{w}(s) \cdot \hat{y}
$$


and observe that

$$
\left|\dot{\beta}_{w}(0)\right|=\tan \Theta .
$$

Note also that $\beta_{w}$ is a constant speed curve; consequently,

$$
d_{\mathcal{E}}\left(\alpha_{w}(t) \cdot \hat{y}, \hat{y}\right)<t(\tan \Theta) .
$$

Substituting (3) into (2) and recalling that $t \leq \mu(\sigma / 2, n)$ yields the first inequality of Lemma 2.

To prove the second inequality, recall that the orbit action is an isometry between $H^{0}$ and $H^{0} \hat{y}$. Hence $|v|=\left|\dot{\alpha}_{v}(0)\right| \geq 1$ since

$$
\left|\dot{\alpha}_{v}(0)\right|=\left|\dot{\beta}_{v}(0)\right| \geq\left|\dot{\beta}_{u}(0)\right|=1 .
$$

Next consider the simply connected nilpotent Lie group $H^{0} / C^{0}$ and its oneparameter subgroup $\gamma$ defined by

$$
\gamma(s)=\alpha_{v}(s) C^{0}, \text { for all } s \in \mathbf{R} .
$$

Put the left invariant Riemannian metric on $H^{0} / C^{0}$ such that the quotient map $H^{0} \rightarrow H^{0} / C^{0}$ is a Riemannian submersion; hence,

$$
|\dot{\gamma}(0)| \geq 1
$$

because of (4) and the fact that $\dot{\alpha}_{v}(0)$ is perpendicular to $T_{e} C^{0}$. (We use $e$ to denote the identity element of a group.) Also note that

$$
d_{H \hat{y}}\left(\alpha_{v}(t) \hat{y}, \hat{y}\right) \geq d_{H^{0} / C^{0}}\left(\gamma(t), C^{0}\right)
$$

since $d_{H \hat{y}}\left(\alpha_{v}(t) \hat{y}, \hat{y}\right)=d_{H}\left(\alpha_{v}(t), e\right)$ and $H^{0} \rightarrow H^{0} / C^{0}$ is a Riemannian submersion. Next observe that the sectional curvatures of $H^{0} / C^{0}$ are bounded above by a positive real number $k\left(n, \epsilon, A_{0}\right)$. This observation follows from Lemma in the above Remarks on Step 10 and from [4, 6.1.8, 7.21]. Therefore Claim 2.8.5, together with Lemma in Remarks on Step 10, and this bound on the sectional curvatures of $H^{0} / C^{0}$ yield that there exists a positive real number $c\left(n, \epsilon, A_{0}\right)$ such that either

$$
\begin{aligned}
& d_{H^{0} / C^{0}}\left(\gamma(t), C^{0}\right) \geq c\left(n, \epsilon, A_{0}\right) \text { or }
\end{aligned}
$$

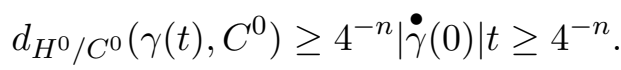

Now define the number $d\left(n, \epsilon, A_{0}\right)$ posited in Lemma 2 by

$$
d\left(n, \epsilon, A_{0}\right)=\min \left\{c\left(n, \epsilon, A_{0}\right), 4^{-n}\right\} .
$$

Then combining the inequalities occurring in (6) and (7) proves the second assertion of Lemma 2.

This completes the proof of Lemma 2. 
Lemma 3. There exist positive real numbers $\nu=\nu\left(n, \epsilon, A_{0}\right)$ and $\tau=$ $\tau\left(n, \epsilon, A_{0}\right)$ such that, for any $z \in \hat{H} \hat{y}$,

$$
d_{H^{0} \hat{y}}(z, \hat{y}) \leq \tau d_{\mathcal{E}}(z, \hat{y})
$$

whenever $d_{\mathcal{E}}(z, \hat{y}) \leq \nu$.

Proof. This is a routine consequence of the following two facts:

1) The normal injectivity radius of $\hat{H} \hat{y}$ in $\mathcal{E}$ is bounded below by a positive number which depends only on $n, \epsilon, A_{0}$.

2) The norm of the second fundamental form for $\hat{H} \hat{y}$ in $\mathcal{E}$ is bounded above by a positive number which depends only on $n, \epsilon, A_{0}$.

These two facts are proven in [4] (cf. 7.21, 6.1.2, 6.1.4 and 6.1.8; also the proof of Proposition A.2.2). This completes the proof for Lemma 3.

Proof of Theorem 1. Set $\sigma=\min \{\nu, d / \tau\}$ and select $\hat{\Theta}$ to be any positive number such that

$$
\mu(\sigma / 2, n) \tan \hat{\Theta}<\sigma / 2 .
$$

(Here $\nu, d, \tau$ and $\mu$ come from Lemmas 1, 2 and 3.) Now suppose the assertion in Theorem 1 is false. Then there exists vectors $u$ and $v$ as in the setup to Lemma 2 such that

$$
\tan \Theta<\tan \hat{\Theta} .
$$

Set $z=\alpha_{v}(t) \hat{y}$ in Lemma 3, where $t$ comes from Lemma 2. Then the first inequality in Lemma 2 yields that

$$
d_{\mathcal{E}}(z, \hat{y})<\sigma<\nu
$$

Hence Lemma 3 applies to show that

$$
d_{H^{0} \hat{y}}(z, \hat{y}) \leq \tau d_{\mathcal{E}}(z, \hat{y}) .
$$

Combining inequalities (1) and (2) with the second inequality in Lemma 2 yields that

$$
d<\tau \sigma
$$

contradicting the fact that $\sigma \leq d / \tau$.

This completes the Proof of Theorem 1.

Continuing now with the verification of Step 2 we will need the following notation:

Notation. Let $G \times X \rightarrow X$ be a smooth action of a Lie group $G$ on a smooth manifold $X$ and $v \in \mathfrak{g}(G)$ where $\mathfrak{g}(G)$ denotes the Lie algebra of $G$. Then $\check{v}$ denotes the vector field on $X$ whose value at $x \in X$ is the tangent vector to the curve

$$
t \rightarrow \alpha_{v}(t) x
$$


at $t=0$. (As usual $\alpha_{v}$ denotes the one-parameter subgroup of $G$ corresponding to $v$.)

Lemma 4. There exists a positive number $K_{1}=K_{1}\left(n, \epsilon, A_{0}\right)$ such that

$$
\left|\hat{D}_{X} \check{v}\right| \leq K_{1}|X||\check{v}|
$$

for ever pair of vectors $v \in \mathfrak{g}\left(H^{0}\right)$ and $X$ tangent to $\mathcal{E}$. (Here $\hat{D}$ denotes the Levi-Civita connection on $\mathcal{E}$.)

Proof. This follows from [4, 4.7 and 4.9].

Recall $\hat{\rho}: \mathcal{E} \rightarrow \hat{V}$ denotes the orbit map. (Also recall $\hat{V}=\mathcal{E} / O(n)$.) And let $D$ denote the Levi-Civita connection on $\hat{V}$. We say that $v \in \mathfrak{g}\left(H^{0}\right)$ is perpendicular to $\mathfrak{g}\left(C^{0}\right)$ at $\hat{y} \in \mathcal{E}$ provided $\check{v}$ is perpendicular to $\check{u}$ at $\hat{y}$ for every vector $u \in \mathfrak{g}\left(C^{0}\right)$.

Theorem 2. There exists a positive number $K_{2}=K_{2}\left(n, \epsilon, A_{0}\right)$ such that

$$
\left|D_{X} \check{v}\right| \leq K_{2}|X||\check{v}|
$$

for every vector $X \in T_{y}(\hat{V})$ and every $v \in \mathfrak{g}\left(H^{0}\right)$ which is perpendicular to $\mathfrak{g}\left(C^{0}\right)$ at $\hat{y} .($ Recall $y=\hat{\rho}(\hat{y})$.

Proof. Since $\hat{\rho}: \mathcal{E} \rightarrow \hat{V}$ is $H^{0}$-equivariant, the vector field $\check{v}$ on $\mathcal{E}$ maps to the vector field $\check{v}$ on $\hat{V}$ via $d \hat{\rho}$; i.e., they are $\hat{\rho}$-related vector fields. Let $\bar{v}$ denote the horizontal lift of $\check{v}$ in $\hat{V}$ to $\mathcal{E}$. Since $\check{v}$ in $\mathcal{E}$ is $\hat{\rho}$-related to $\check{v}$ in $\hat{V}$, $\bar{v}$ is the horizontal component of $\check{v}$ in $\mathcal{E}$; i.e.,

$$
\check{v}=\bar{v}+w
$$

where $w$ is a vertical vector field in $\mathcal{E}$ relative to the Riemannian submersion $\hat{\rho}: E \rightarrow \hat{V}$. Let $\bar{X}$ denote the horizontal lift of $X$ to $\mathcal{E}$, then

$$
\hat{D}_{\bar{X}} \bar{v}=\hat{D}_{\bar{X}} \check{v}-\hat{D}_{\bar{X}} w
$$

because of (1). Consequently

$$
\mathcal{H}\left(\hat{D}_{\bar{X}} \bar{v}\right)=\mathcal{H}\left(\hat{D}_{\bar{X}} \check{v}\right)-\mathcal{H}\left(\hat{D}_{\bar{X}} w\right)
$$

where $\mathcal{H}(u)$ denotes the horizontal component of a vector $u$ tangent to $\mathcal{E}$. By a standard result (cf. [16, p. 212, Lemma 45])

$$
\mathcal{H}\left(\hat{D}_{\bar{X}} \bar{v}\right)=\bar{Y} \text { where } Y=\left(D_{X} \check{v}\right) \text {. }
$$

Applying this fact and Lemma 4 to (3) yields

$$
\left|D_{X} \check{v}\right| \leq K_{1}|X|\|\check{v}\|+\left|\mathcal{H}\left(\hat{D}_{\bar{X}} w\right)\right|
$$

where $\|\check{v}\|$ denotes the length of $\check{v}$ in $\mathcal{E}$. Let $\omega$ denote the angle between $\check{v}$ and $\bar{v}$ at $\hat{y}$, then

$$
(\pi / 2-\omega)>\hat{\Theta}
$$


because of Theorem 1 and the fact that $v$ is perpendicular to $\mathfrak{g}\left(C^{0}\right)$ at $\hat{y}$. (Recall that $\hat{\Theta}=\hat{\Theta}\left(n, \epsilon, A_{0}\right)>0$.) Consequently,

$$
\|\check{v}\| \leq(\csc \hat{\Theta})|\check{v}| \text {. }
$$

Combining inequalities (4) and (6) yields

$$
\left|D_{X} \check{v}\right| \leq(\csc \hat{\Theta}) K_{1}|X \| \check{v}|+\left|\mathcal{H}\left(\hat{D}_{\bar{X}} w\right)\right| .
$$

It remains to estimate $\left|\mathcal{H}\left(\hat{D}_{\bar{X}} w\right)\right|$. For this purpose, a routine application of the Koszul formula (as in the proof of Lemma A.2.5.4(c) above) yields that

$$
\hat{D}_{\bar{X}} w \cdot \bar{Y}=\Omega(\bar{X}, \bar{Y}) \cdot \check{u}
$$

where $Y$ is any vector tangent to $\hat{V}$ at $y ; u \in \mathfrak{g}(O(n))$ is the unique vector such that $\check{u}=w$ at $\hat{y}$, and $\Omega($, ) denotes the curvature form for the principal $O(n)$-bundle $\hat{\rho}: \mathcal{E} \rightarrow \hat{V}$ relative to its horizontal distribution.

Claim 1. The norm of $\Omega(, \quad)$ is bounded above by a positive number $K_{0}=K_{0}\left(n, \epsilon, A_{0}\right)$.

Before verifying this claim, we us it to complete the proof of Theorem 2. (Its verification will come at the end of Remarks on Step 2.) Claim 1 applied to Equation (8) yields

$$
\left|\mathcal{H}\left(\hat{D}_{\bar{X}} w\right)\right| \leq K_{0}|X||w| .
$$

Now note that inequality (5) yields

$$
|w|<(\cot \hat{\Theta})|\check{v}| .
$$

Combining inequalities (7), (9) and (10) yields that

$$
\left|D_{X} \check{v}\right| \leq\left(K_{1} \csc \hat{\Theta}+K_{0} \cot \hat{\Theta}\right)|X||\check{v}|
$$

proving Theorem 2.

Lemma 5. Let $v \in \mathfrak{g}\left(H^{0}\right)$ and $u \in T_{y}(\hat{V})$, then

$$
D_{\check{v}} \hat{u}=D_{u} \check{v} \text {. }
$$

Remark. Recall $\hat{u}$ denotes the $H$-invariant vector field along $H y$ whose value at $y$ is $u$.

Proof. This is because $[\hat{u}, \check{v}]=0$, which is seen by considering the parametrized surface $f: \mathbf{R}^{2} \rightarrow \hat{V}$ defined by

$$
f(s, t)=\alpha_{v}(t) \beta(s)
$$


where $\beta: \mathbf{R} \rightarrow \hat{V}$ is any smooth curve such that $\dot{\beta}(0)=u$. Then note that $\partial f / \partial s=\hat{u}$, while $\partial f / \partial t=\check{v}$. This completes the proof of Lemma 5 .

We are now ready to show that the angle between $H \bar{y}$ and $\bar{y} O(n)$ at $\bar{y} \in E$ is bounded by a positive number $\bar{\Theta}=\bar{\Theta}\left(n, \epsilon, A_{0}\right)$. To do this, let $v \in \mathfrak{g}\left(H^{0}\right)$ and define smooth curves $\beta: \mathbf{R} \rightarrow E$ and $\gamma: \mathbf{R} \rightarrow \hat{V}$ by the equations

$$
\beta(t)=\alpha_{v}(t) \bar{y} \text { and } \gamma(t)=\alpha_{v}(t) y
$$

for all $t \in \mathbf{R}$. Note that $\gamma=\rho \circ \beta$ and that $\dot{\gamma}(0)=\check{v}$ in the notation fixed in Lemma 4. Assume also that $v$ is perpendicular to $\mathfrak{g}\left(C^{0}\right)$ at $\hat{y} \in \mathcal{E}$. Recall that $\bar{y}$ is an orthonormal framing of $T_{y}(\hat{V})$; i.e.,

$$
\bar{y}=\left(u_{1}, u_{2}, \ldots, u_{n}\right)
$$

where each $u_{i} \in T_{y}(\hat{V})$ and

$$
u_{i} \cdot u_{j}= \begin{cases}1 & \text { if } i=j \\ 0 & \text { if } i \neq j\end{cases}
$$

Hence $\dot{\beta}(0) \in T_{\bar{y}}(E)$ is represented by the following $(n+1)$-tuple of vectors in $T_{y}(\hat{V})$ :

$$
\left(\check{v}, D_{\check{v}} \hat{u}_{1}, D_{\check{v}} \hat{u}_{2}, \ldots, D_{\check{v}} \hat{u}_{n}\right)
$$

which equals

$$
\left(\check{v}, D_{u_{1}} \check{v}, D_{u_{2}} \check{v}, \ldots, D_{u_{n}} \check{v}\right)
$$

because of Lemma 5. Applying Theorem 2, we see that

$$
\left|D_{u_{i}} \check{v}\right| \leq K_{2}|\check{v}| \text { for } i=1,2, \ldots, n \text {. }
$$

Let $\omega$ denote the angle between $\dot{\beta}(0)$ and the $O(n)$-orbit $\bar{y} O(n)$ at $\bar{y}$, then $(* * *)$

$$
\tan \omega \geq 1 /\left(n K_{2}\right)
$$

because of $(*)$ and $(* *)$. It is now a routine exercise, using $(* * *)$, to show that the angle between the tangent spaces to $H \bar{y}$ and $\bar{y} O(n)$ at $\bar{y}$ is bounded from below by a number $\bar{\Theta}=\bar{\Theta}\left(n, \epsilon, A_{0}\right)>0$. This completes the demonstration of Step 2 modulo the verification of Claim 1.

\section{Verification of Claim 1.}

We start by stating the following extension of Lemma A.2.5.4 which follows again from a routine application of the Koszul formula:

Observation. Let $p: \mathbb{E} \rightarrow N$ be any principal $O(n)$-bundle where $O(n)$ acts by isometries on the Riemannian manifold $\mathbb{E}$ and $p$ is a Riemannian submersion. Then formulas (b) and (c) of Lemma A.2.5.4 remain true in 
this more general setting where $X, Y, Z$ are now vector fields on $N$ and special vector fields $\check{u}, \check{v}, \check{w}, \bar{X}, \bar{Y}, \bar{Z}$ are defined as in the preamble to A.2.5.4. Furthermore, formulas (a) and (d) also hold true if the right invariant Riemannian metric induced on $O(n)$ by the immersion $g \rightarrow z g, g \in O(n)$, and $z$ a fixed point in $\mathbb{E}$, is independent of $z$ and is identical to that determined by the negative of the Killing form for the Lie algebra $\mathfrak{g}(O(n))$; cf. Remark A.2.5.2.1.

Let $\Omega$ and $\hat{\Omega}$ denote the curvature forms for the principal $O(n)$-bundle

$$
\hat{\rho}: \mathcal{E} \rightarrow \hat{V}
$$

relative to the horizontal distributions determined by the $O(n)$-invariant Riemannian metrics $\widetilde{h}_{\epsilon}$ and $\widetilde{h}$ on $\mathcal{E}$, respectively. Here $\widetilde{h}_{\epsilon}$ and $\widetilde{h}$ are as in Proposition 7.2.1 of [4] and $h$ is the Riemannian metric $S_{\epsilon / 2}(g)$ of $[4$, p. 362, line-5]. Also $g_{\epsilon}$ denotes the Riemannian metric on $\hat{V}$ such that

$$
\hat{\rho}:\left(\mathcal{E}, \widetilde{h}_{\epsilon}\right) \rightarrow\left(\hat{V}, g_{\epsilon}\right)
$$

is a Riemannian submersion.

Because the standard relation between $\hat{\Omega}$ and the curvature tensor on $\hat{V}$ for the Riemannian metric $h$, given in $[\mathbf{1 5}$, Vol.1, p. 133], we see that the norm of $\hat{\Omega}$ is bounded above by a positive number

$$
\hat{K}_{0}=\hat{K}_{0}\left(n, \epsilon, A_{0}\right) \text {. }
$$

So it suffices to show that the norms of $\Omega$ and $\hat{\Omega}$ are nicely related.

To do this, we use the notation $\bar{X}$ and $\hat{X}$ for the horizontal lifts of a vector (or vector field) $X$ in $\hat{V}$ to $\mathcal{E}$ with reference to the horizontal distributions determined by $\widetilde{h}_{\epsilon}$ and $\widetilde{h}$, respectively. Let $X$ and $Y$ be vectors tangent to $\hat{V}$ based at a common point and such that

$$
g_{\epsilon}(X, X)=g_{\epsilon}(Y, Y)=1
$$

and let $u \in \mathfrak{g}(O(n))$ denote $\Omega(\bar{X}, \bar{Y})$ where the horizontal lifts $\bar{X}, \bar{Y}$ are also based at a common point in $\mathcal{E}$. Recall that $u$ is the unique vector satisfying

$$
\check{u}=2^{-1}[\bar{Y}, \bar{X}] .
$$

We proceed to estimate $|u|$ where || denotes the norm on $\mathfrak{g}(O(n))$ determined by the negative of its Killing form; cf. Remark A.2.5.2.1. First note that

$$
|u|^{2} \leq 2 \widetilde{h}_{\epsilon}(\check{u}, \check{u})
$$

since $|u|^{2}=\widetilde{h}(\check{u}, \check{u})$ and because of the inequality (7.21.1) of [4]. (Here and throughout the proof of Claim 1 we assume that $\epsilon$ is sufficiently small relative to the number 2.)

Let $\bar{D}$ and $\hat{D}$ denote the Levi-Civita connections on $\mathcal{E}$ with respect to $\widetilde{h}_{\epsilon}$ and $\widetilde{h}$, then

$$
\widetilde{h}_{\epsilon}(\check{u}, \check{u})=\widetilde{h}_{\epsilon}\left(\bar{D}_{\bar{X}} \check{u}, \bar{Y}\right)
$$


because of formula (c) of A.2.5.4 and Equation (1); cf. Observation. But note that

$$
\widetilde{h}_{\epsilon}\left(\bar{D}_{\bar{X}} \check{u}, \bar{Y}\right) \leq 2\left|\widetilde{h}\left(\hat{D}_{\bar{X}} \check{u}, \bar{Y}\right)\right|+2|u|
$$

because of inequality (7.21.1) of [4]. Let $v, w \in \mathfrak{g}(O(n))$ denote the unique vectors such that

$$
\begin{aligned}
& \bar{X}=\hat{X}+\check{v}, \\
& \bar{Y}=\hat{Y}+\check{w} .
\end{aligned}
$$

It follows that

$$
|v|,|w|, h(X, X), h(Y, Y) \leq 2
$$

because of Equations (0) and inequality (7.21.1) of [4]. Expanding $\widetilde{h}\left(\hat{D}_{\bar{X}} \check{u}, \bar{Y}\right)$ using Equations (5) yields that

$$
\widetilde{h}\left(\hat{D}_{\bar{X}} \check{u}, \bar{Y}\right)=\widetilde{h}\left(\hat{D}_{\hat{X}} \check{u}, \hat{Y}\right)+\widetilde{h}\left(\hat{D}_{\check{v}} \check{u}, \check{w}\right)+\widetilde{h}\left(\hat{D}_{\hat{X}} \check{u}, \check{w}\right)+\widetilde{h}\left(\hat{D}_{\check{v}} \check{u}, \hat{Y}\right) .
$$

And applying formulas (a), (c) and (d) of Lemma A.2.5.4 to Equation (7) yields

$$
\widetilde{h}\left(\hat{D}_{\bar{X}} \check{u}, \bar{Y}\right)=\hat{\Omega}(\hat{X}, \hat{Y}) \cdot u-2^{-1}[u, v] \cdot w .
$$

(Formulas (a) and (d) hold because $\widetilde{h}$ is the naturally induced Riemannian metric on the principle tangent bundle of the Riemannian manifold $(\hat{V}, g)$; cf. Observation.) Combining formulas (2), (3), (4) and (8) and using the fact that the inner product on $\mathfrak{g}(O(n))$ is bi-invariant yields that

$$
|u|^{2} \leq 4|\hat{\Omega}(\hat{X}, \hat{Y}) \| u|+2|[v, w]||u|+4|u| .
$$

Canceling $|u|$ from inequality (9) and using that the norm of $\hat{\Omega}$ is bounded above by $\hat{K}_{0}$ together with the inequalities (6) yields that

$$
|u| \leq 8 \hat{K}_{0}+8 \eta+4
$$

where $\eta$ is the norm of the Lie bracket on $\mathfrak{g}(O(n))$. This inequality (10) shows that we can set $K_{0}=8 \hat{K}_{0}+8 \eta+4$ completing the verification of Claim 1.

We end these Remarks on Step 2 by fulfilling the promise made in "Remarks on Steps 1 and 4" to give an alternate verification of inequality (8.7) in [4]. This inequality follows directly from the following three results proven above: Theorem 2, Lemma 5, and the fact that the angle between the tangent spaces to $H \bar{y}$ and $\bar{y} O(n)$ at $\bar{y}$ is bounded below by the positive number $\bar{\Theta}\left(n, \epsilon, A_{0}\right)$.

Remarks on Fact 1. To complete the Proof of Theorem A.2.3 it remains to verify Fact 1 . We start our verification of Fact 1 with the following result: 
Lemma 1. Let $T$ be a maximal torus in $O(n)$. Given $v>0$, there exists only a finite number of closed connected subgroups $S$ of $T$ such that $\operatorname{Vol}(S) \leq v$.

Proof. Let $m=\operatorname{dim} T$ and $s=\operatorname{dim} S$, then $s \in\{0,1,2, \ldots, m\}$. Hence in proving that the number of such subgroups $S$ is finite, we may assume that $s$ is fixed and $s \neq 0$. Let $M^{*}(m, s ; \mathbb{Z})$ denote the set of all $m \times s$ matrices $A$ with integral entries and $\operatorname{rank} A=s$. Also let $T^{i}$ denote the Lie group $S^{1} \times S^{1} \times \ldots \times S^{1}$ (i-factors) and identify $T$ with $T^{m}$. Then each $A \in M^{*}(m, s ; \mathbb{Z})$ determines a homomorphism $f_{A}: T^{s} \rightarrow T$. Note that if $B \in M^{*}(s, s ; \mathbb{Z})$, then image $\left(f_{A B}\right)=\operatorname{image}\left(f_{A}\right)$. Furthermore for each closed connected subgroup $S$ of $T$, there is a matrix $A \in M^{*}(m, s ; \mathbb{Z})$ such that both image $\left(f_{A}\right)=S$ and $f_{A}$ is monic. An elementary argument yields the following estimate for $\operatorname{Vol}(S)$. There is a positive constant $\sigma$ (independent of $S$ ) such that

$$
\operatorname{Vol}(S) \geq \sigma|\operatorname{det}| /\left|\operatorname{ker}\left(f_{A}\right)\right|
$$

where "det" denotes the determinant of any $s \times s$ submatrix of $A \in M^{*}(m, s$; $Z$ ) and image $\left(f_{A}\right)=S$. Since rank $A=s$, there exists an $s \times s$ submatrix $B$ of $A$ such that det $B \neq 0$; i.e., $B \in M^{*}(s, s ; Z)$. Let $D=A \operatorname{adj}(B)$ where $\operatorname{adj}(B)$ denotes the classical adjoint of $B$. Since image $\left(f_{D}\right)=$ image $\left(f_{A}\right)$, to prove Lemma 1, it suffices to show that the absolute values of the entries in $D$ are all bounded above by a positive number $K=K(v, m)$, where $v$ is any upper bound for $\operatorname{Vol}\left(f_{A}\right)$, when $f_{A}$ is monic. But this follows from inequality $(*)$, in which $D$ replaces $A$, by considering the different $s \times s$ submatrices of $D$. In fact, we can take

$$
K(v, m)=v / \sigma .
$$

This completes the proof of Lemma 1.

Lemma 2. Given $v>0$, there are only finitely many conjugacy classes of closed subgroups $C$ of $O(n)$ such that both $C^{0}$ is abelian and $\operatorname{Vol}(C) \leq v$.

Proof. Fix a maximal torus $T$ in $O(n)$. Then $C^{0}$ is conjugate to a subgroup of $T$. Hence Lemma 1 shows that there exists a positive integer $\tau=\tau(n, v)$ such that the finite subgroup $C / C^{0}$ has order $\leq \tau$. Another consequence of Lemma 1 is that it suffices, in proving Lemma 2, to demonstrate the following weaker statement:

(*) Given $v>0$ and a closed connected abelian subgroup $S$ of $O(n)$, there are only finitely many conjugacy classes of closed subgroups $C$ of $O(n)$ such that both $C^{0}=S$ and $\operatorname{Vol}(C) \leq v$.

To verify statement $(*)$, consider the normalizer $N(S)$ of $S$ in $O(n)$ and note that $N(S)$ is a compact Lie group and that $C$ is a subgroup of $N(S)$. Consider the factor Lie group $N(S) / S$ and its finite subgroup $C / S$ whose 
order is less than $\tau$. Then $(*)$ is an easy consequence of the following observation:

(**) There are only finitely many conjugacy classes of finite subgroups of $N(S) / S$ of order $\leq \tau$.

Observation $(* *)$ is a consequence of the fact that representations of finite groups into Lie groups are (locally) rigid, because of Weil's rigidity theorem, together with the fact that $N(S) / S$ is compact.

This completes the proof of Lemma 2.

Lemma 3. There is a function $f: \mathbf{R}^{+} \times \mathbb{Z}^{+} \rightarrow \mathbf{R}^{+}$for which the following statement is true. Let $C$ be any closed subgroup of $O(n)$ such that both $C^{0}$ is abelian and

$$
\operatorname{Vol}\left(C \backslash S^{n-1}\right) \geq v>0,
$$

then

$$
\operatorname{Vol}(C) \leq f(v, n)
$$

Remark. The function $f$ in Lemma 3 can in fact be taken to be

$$
f(v, n)=\operatorname{Vol}(T) \operatorname{Vol}\left(T \backslash S^{n-1}\right) / v
$$

where $T$ is a maximal torus in $O(n)$. (Since all maximal tori in $O(n)$ are conjugate, this function depends only on $v$ and $n$.)

Proof. Let $P(C)$ denote the set of all points in $S^{n-1}$ at which $C$ acts freely. Then $P(C)$ is an open dense submanifold of $S^{n-1}$; in fact,

$$
\operatorname{Vol}(P(C))=\operatorname{Vol}\left(S^{n-1}\right) \text {. }
$$

Put the Riemannian metric on $C \backslash P(C)$ such that the orbit map $P(C) \rightarrow$ $C \backslash P(C)$ is a Riemannian submersion. Let $\mu_{C}$ be the measure on $C \backslash P(C)$ determined by this Riemannian metric, then

$$
\operatorname{Vol}\left(C \backslash S^{n-1}\right)=\mu_{C}(C \backslash P(C))
$$

and

$$
\operatorname{Vol}\left(S^{n-1}\right)=\int_{C x \in C \backslash P(C)} \operatorname{Vol}(C x) d \mu_{C} .
$$

Special Case. We first verify Lemma 3 under the extra assumption that $C$ is connected; i.e., $C=C^{0}$. Then let $T$ be a maximal torus in $O(n)$ containing $C$. Note that $P(T) \subset P(C)$ and

$$
\operatorname{Vol}(C \backslash P(C))=\operatorname{Vol}(C \backslash P(T))
$$

since $\operatorname{Vol}(P(C))=\operatorname{Vol}(P(T))$. Consider the principal bundle

$$
C \backslash T \rightarrow C \backslash P(T) \stackrel{p}{\longrightarrow} T \backslash P(T)
$$


and notice that $p$ is a Riemannian submersion since $C \backslash T$ acts by isometries on $C \backslash P(T)$. Our argument in this special case is based on the following assertion:

Claim. For each point $C x \in C \backslash P(T)$, the following inequality is true:

$$
\operatorname{Vol}(C \backslash T(C x)) \leq \operatorname{Vol}(T) / \operatorname{Vol}(C) .
$$

Proof of Claim. Note first that

$$
C \backslash T(C x)=q(T x)
$$

where $q: P(T) \rightarrow C \backslash P(T)$ is the orbit map. Also note that

$$
\operatorname{Vol}(C(t x))=\operatorname{Vol}(C x)
$$

for each $t \in T$; since $T$ is abelian and $C \subset T$. Hence

$$
\operatorname{Vol}(T x)=\operatorname{Vol}(C x) \operatorname{Vol}(q(T x))
$$

since $q$ is a Riemannian submersion; i.e.,

$$
\operatorname{Vol}(C \backslash T(C x))=\operatorname{Vol}(T x) / \operatorname{Vol}(C x) .
$$

Consider the Riemannian submersion $f: O(n) \rightarrow S^{n-1}$ defined by $f(g)=$ $g x, g \in O(n)$. Note that $f$ maps both $T$ and $C$ diffeomorphically onto $T x$ and $C x$, respectively, since $x \in P(T)$. Let $\zeta$ denote $d f: \mathfrak{g}(C) \rightarrow T_{x}(C x)$ and $\xi$ denote $d f: \mathfrak{g}(T) \rightarrow T_{x}(T x)$. Then note that

$$
\begin{aligned}
& \operatorname{Vol}(C x)=|\operatorname{det} \zeta| \operatorname{Vol}(C) \text { and } \\
& \operatorname{Vol}(T x)=|\operatorname{det} \xi| \operatorname{Vol}(T)
\end{aligned}
$$

where $\operatorname{det} \zeta$ and $\operatorname{det} \xi$ are computed using orthonormal bases for the relevant vector spaces; therefore, we obtain by dividing these equations that

$$
\operatorname{Vol}(T x) / \operatorname{Vol}(C x)=|\operatorname{det} \xi| \operatorname{Vol}(T) /|\operatorname{det} \zeta| \operatorname{Vol}(C) \text {. }
$$

Concatenating Equations (2) and (3) establishes the Claim once we verify that

$$
|\operatorname{det} \xi| \leq|\operatorname{det} \zeta| \text {. }
$$

But inequality (4) is true since $\xi$ is a weakly decreasing linear transformation; i.e.,

$$
|\xi(v)| \leq|v|
$$

for all $v \in \operatorname{domain}(\xi)$. This completes the verification of Claim.

We now complete the proof of the Special Case of Lemma 3. Recall that the projection $p$ in the principal bundle of $(* *)$ is a Riemannian submersion; hence,

$$
\operatorname{Vol}(C \backslash P(T))=\int_{T x \in T \backslash P(T)} \operatorname{Vol}(C \backslash T(C x)) d \mu_{T}
$$


Combining Equations (1) and (5) with the Claim and the facts that

$$
\begin{aligned}
& \operatorname{Vol}\left(C \backslash S^{n-1}\right)=\operatorname{Vol}(C \backslash P(C)) \text { and } \\
& \operatorname{Vol}\left(T \backslash S^{n-1}\right)=\operatorname{Vol}(T \backslash P(T))
\end{aligned}
$$

yield the following inequality:

$$
\operatorname{Vol}\left(C \backslash S^{n-1}\right) \leq(\operatorname{Vol}(T) / \operatorname{Vol}(C)) \operatorname{Vol}\left(T \backslash S^{n-1}\right) .
$$

Note that inequality (6) is equivalent to

$$
\operatorname{Vol}(C) \leq \operatorname{Vol}(T) \operatorname{Vol}\left(T \backslash S^{n-1}\right) / \operatorname{Vol}\left(C \backslash S^{n-1}\right) .
$$

But inequality $(7)$ shows that the function $f(v, n)$ defined in Remark satisfies Lemma 3 in the special case.

We now prove Lemma 3 in general; i.e., without assuming $C=C^{0}$. Let $T$ be a maximal torus in $O(n)$ containing $C^{0}$ and $G$ be the finite group $C / C^{0}$ whose order is denoted by $|G|$. Note that inequality (7) is valid when we replace $C$ by $C^{0}$; i.e.,

$$
\operatorname{Vol}\left(C^{0}\right) \leq \operatorname{Vol}(T) \operatorname{Vol}\left(T \backslash S^{n-1}\right) / \operatorname{Vol}\left(C^{0} \backslash S^{n-1}\right) .
$$

Also note that

$$
\operatorname{Vol}\left(C^{0} \backslash S^{n-1}\right)=|G| \operatorname{Vol}\left(C \backslash S^{n-1}\right) .
$$

This is because

$$
\operatorname{Vol}\left(C^{0} \backslash S^{n-1}\right)=\operatorname{Vol}\left(C^{0} \backslash P\left(C^{0}\right)\right)=\operatorname{Vol}\left(C^{0} \backslash P(C)\right)
$$

and

$$
\operatorname{Vol}\left(C^{0} \backslash P(C)\right)=|G| \operatorname{Vol}(C \backslash P(C)) .
$$

But we obviously have

$$
\operatorname{Vol}(C)=|G| \operatorname{Vol}\left(C^{0}\right)
$$

Substituting Equations (9) and (10) into inequality (8) now yields that inequality (7) is true in general. This shows that the function $f(v, n)$ defined in Remark satisfies Lemma 3 in general, completing the proof for Lemma 3 .

We have the following corollary obtained by directly combining Lemmas 2 and 3 :

Corollary 4. Given $v>0$, there are only finitely many conjugacy classes of closed subgroups $C$ of $O(n)$ such that both $C^{0}$ is abelian and $\operatorname{Vol}\left(C \backslash S^{n-1}\right) \geq$ $v$.

We have now completed discussing the preliminaries needed to verify Fact 1. We've shown that to verify Fact 1 it suffices to show that there exists a positive number $b=b\left(n, \epsilon, A_{0}\right)$ such that

$$
\operatorname{Vol}(\bar{C} \backslash O(n)) \geq b \text {. }
$$


This assertion is an immediate consequence of Corollary 4. Let

$$
d: C \rightarrow \operatorname{Iso}\left(T_{y} \hat{V}\right)
$$

denote the derivative map at $y \in \hat{V}$. (Recall that $C$ is the isotropy subgroup at $y$ of the action of $H$ on $\hat{V}$.) The map $d$ is a faithful representation. Recall that $\bar{y} \in F \hat{V}$ is an orthonormal basis for $T_{y} \hat{V}$ and that, for each $c \in C$, $\bar{c} \in O(n)$ is the matrix representing linear transformation

$$
d(c): T_{y} \hat{V} \rightarrow T_{y} \hat{V}
$$

with respect to this basis. Hence, to verify Fact 1 it suffices to show that

$$
\operatorname{Vol}\left(d(C) \backslash S_{y}\right) \geq b
$$

where $S_{y}$ denotes the sphere of radius 1 in $T_{y} \hat{V}$.

Recall again the Riemannian manifold $\mathcal{E}$ used in $[4, \S 8]$ to construct $\hat{V}$. It has an action of $\hat{H} \times O(n)$ such that $\hat{V}=\mathcal{E} / O(n)$ and there is a point $\hat{y} \in \mathcal{E}$ such that $y$ is the orbit $\hat{y} O(n)$. There is also an isomorphism $\alpha$ which maps $\hat{C}$ onto a subgroup $\widetilde{C}$ of $O(n)$ defined by the equation

$$
g \hat{y}=\hat{y} \alpha(g)
$$

for each $g \in \hat{C}$. (Recall that $\hat{C}=\phi^{-1}(C)$ where $\phi: \hat{H} \rightarrow H$ is an epimorphism such that the principal $O(n)$-bundle projection

$$
\hat{\rho}: \mathcal{E} \rightarrow \hat{V}
$$

is $\phi$-equivariant; cf. paragraph 2 of Remarks to Step 2.) Let $z$ denote the orbit $\hat{H} \hat{y}$ in $\hat{H} \backslash \mathcal{E}$ and $S_{z}$ denote the sphere of radius 1 in $T_{z}(\hat{H} \backslash \mathcal{E})$. Then it is easy to construct an $\alpha$-equivariant isometry from $S_{y}$ to $S_{z}$ where $\hat{C}$ acts on $S_{y}$ via the composite representation $d \circ \phi$. Hence

$$
\operatorname{Vol}\left(d(C) \backslash S_{y}\right)=\operatorname{Vol}\left(d(\widetilde{C}) \backslash S_{z}\right)
$$

where again $d: \widetilde{C} \rightarrow$ Iso $\left(T_{z}(\hat{H} \backslash \mathcal{E})\right)$ denotes the derivative map at $z \in \hat{H} \backslash \mathcal{E}$. But

$$
\operatorname{Vol}\left(d(\widetilde{C}) \backslash S_{z}\right) \geq b
$$

because of [4, Lemma 8.5].

This completes the verification of Fact 1.

\section{References}

[1] J. Bemelmans, M. Min-Oo and E. Ruh, Smoothing Riemannian metrics, Math. Zeitschrift, 188 (1984), 69-74, MR 85m:58184, Zbl 0536.53044.

[2] P. Buser and H. Karcher, Gromov's almost flat manifolds, Asterisque, 81, Societe Mathematique de France, 1981, MR 83m:53070, Zbl 0459.53031.

[3] J. Cheeger and D. Ebin, Comparison Theorems in Riemannian Geometry, NorthHolland Publ., Amsterdam, 1975, MR 56 \#16538, Zbl 0309.53035. 
[4] J. Cheeger, K. Fukaya and M. Gromov, Nilpotent structures and invariant metrics on collapsed manifolds, J. Amer. Math. Soc., 5 (1992), 327-372, MR 93a:53036, Zbl 0758.53022.

[5] J. Cheeger and M. Gromov, Collapsing Riemannian manifolds while keeping their curvature bounded I, J. Differential Geom., 23 (1986), 309-346, MR 87k:53087, Zbl 0606.53028.

[6] _ Collapsing Riemannian manifolds while keeping their curvature bounded II, J. Differential Geom., 32 (1990), 269-298, MR 92a:53066, Zbl 0727.53043.

[7] F.T. Farrell and L.E. Jones, Foliated control without radius of injectivity restrictions, Topology, 30 (1991), 117-142, MR 92d:57025, Zbl 0728.57018.

[8] _ Rigidity for aspherical manifolds with $\pi_{1}$ contained in $\mathrm{GL}_{m}(\mathbb{R})$, Asian Journal of Math., 2 (1998), 215-262, MR 99j:57032.

[9] _ Collapsing foliated Riemannian manifolds, Asian Journal of Math., 2 (1998), 443-494, MR 20001f:53080, Zbl 0982.53030.

[10] K. Fukaya, Collapsing Riemannian manifolds to ones of lower dimension, J. Differential Geom., 25 (1987), 139-156, MR 88b:53050, Zbl 0606.53027.

[11] _ Collapsing Riemannian manifolds to ones of lower dimension II, J. Math. Soc. Japan, 41 (1989), 333-356, MR 90c:53103, Zbl 0703.53042.

[12] S. Helgason, Differential Geometry of Symmetric Spaces, New York, Academic Press, 1962, MR 26 \#2986, Zbl 0111.18101.

[13] N.J. Hicks, Notes on Differential Geometry, Van Nostrand Reinhold Co., London, 1965, MR 31 \#3936, Zbl 0132.15104.

[14] J. Jost and H. Karcher, Geometrische methoden zur Gewinnug von a-priori-Schranker fur harmonische Abbildungen, Manuscripta Math., 40 (1982), 27-77, MR 84e:58023, Zbl 0502.53036.

[15] S. Kobayashi and K. Nomizu, Foundations of Differential Geometry, Vol. 1, Interscience Publishers, New York, 1963, MR 27 \#2945, Zbl 0119.37502.

[16] B. O’Neill, Semi-Riemannian Geometry, Academic Press, New York, 1983, MR 85f:53002, Zbl 0531.53051.

[17] M.S. Raghunathan, Discrete Subgroups of Lie Groups, Springer-Verlag, New YorkHeildelberg-Berlin, 1972, MR 58 \#22394a, Zbl 0254.22005.

Received December 15, 1997 and revised July 27, 2000. Both authors were supported in part by the National Science Foundation.

Department of Mathematics

SUNY AT BINGHAMTON

Binghamton, NY 13902-6000

E-mail address: farrell@math.binghamton.edu

Department of Mathematics

State University of NeW York

STONY BRoOK, NY 11794-3651

E-mail address: lejones@math.sunysb.edu 